\title{
Development of a shear-thinning biomaterial as an endovascular embolic agent for the treatment of type $B$ aortic dissection
}

\author{
Matthew J. Moore ${ }^{1,2}$ Lauren Malaxos ${ }^{1,3}$ and Barry J. Doyle $1,3,4,5$
}

1. Vascular Engineering Laboratory, Harry Perkins Institute of Medical Research, QEII Medical Centre, Nedlands and Centre for Medical Research, The University of Western Australia, Perth, Australia.

2. School of Biomedical Science, The University of Western Australia, Perth, Australia.

3. School of Engineering, The University of Western Australia, Perth, Australia.

4. Australian Research Council Centre for Personalised Therapeutics Technologies, Australia.

5. BHF Centre for Cardiovascular Science, The University of Edinburgh, Edinburgh, UK.

\section{Corresponding Author:}

Barry Doyle, Tel: +61 86151 1084, Fax: +61 86151 1084, Email: barry.doyle@uwa.edu.au 6 Verdun Street, Nedlands, Perth, WA 6009, Australia.

ORCID ID: 0000-0003-4923-2796 


\begin{abstract}
False lumen embolisation is a promising treatment strategy in type B aortic dissection (TBAD) but it is limited by the lack of a disease-specific embolic agent. Our aim was to develop a biomaterial that could be delivered minimally-invasively into the TBAD false lumen. We created 24 shear-thinning biomaterials from blends of gelatin, silicate nanoparticles and silk fibroin, and evaluated their suitability as a false lumen embolic agent in TBAD. We determined the stability of mechanical properties by measuring the compressive modulus of samples stored in physiological conditions over a 21 day period. We quantified injectability by measuring the force required to inject each material through catheters of varying diameter. We also assessed in vitro degradation rates by measuring weight change over 30 days. Finally, we developed an in vitro experimental pulsatile flow setup with two different anatomically-correct TBAD geometries and performed 78 false lumen occlusion experiments under different operating conditions. We found that compressive moduli changed rapidly on exposure to $37^{\circ} \mathrm{C}$ before stabilising by Day 7. A high silicate nanoparticle to gelatin ratio resulted in greater compressive moduli, with a maximum of $117.6 \pm 15.2 \mathrm{kPa}$. By reducing the total solid concentration, we could improve injectability and materials with $8 \%(\mathrm{w} / \mathrm{v})$ solids required $<80 \mathrm{~N}$ force to be injected through a $4.0 \mathrm{~mm}$ catheter. Our in vitro degradation rates showed that the material only degraded by $1.5-8.4 \%$ over a 30 day period. We found that the biomaterial could occlude flow to the false lumen in $99 \%$ of experiments. In conclusion, blends with high silicate nanoparticle and low silk fibroin content warrant further investigation for their potential as false lumen embolic agents and could be a promising alternative to current TBAD repair methods.
\end{abstract}

Keywords: aortic dissection; mechanical testing; biomaterial; gelatin; silk fibroin; silicate nanoparticles; embolisation 


\section{INTRODUCTION}

Aortic dissection is a serious vascular disease with increasing incidence and poor survival rates [1]. It usually occurs when the intima layer of the aorta fails, allowing blood to leak into the aortic wall. This forces the separation of the media layer, creating a false blood-filled lumen [2]. Aortic dissections are termed Stanford type B aortic dissections (TBAD) when the entry tear originates distal to the left subclavian artery [3]. The current treatment is medical antiimpulse therapy, with surgery reserved for those patients who develop early complications. Complication can be described as rupture, malperfusion, or early false lumen expansion [4].

The preferred surgical intervention is thoracic endovascular aortic repair (TEVAR), which involves covering the primary intimal tear with a stent-graft to prevent blood flow into and through the false lumen [5]. The aim of TEVAR is to depressurise the false lumen to induce aortic remodelling and promote false lumen thrombosis (FLT). Patients who achieve complete FLT have a lower risk of aortic rupture and higher survival rates compared to those with partial or no FLT [6]. However, distal re-entry tears or endoleaks may permit continued flow into the false lumen, creating a persistent risk of aortic rupture. In such cases, a patient's treatment options are limited. Adjunct procedures to directly occlude the false lumen by metallic coil embolisation have been attempted [7], though coils can cause significant streak artefacts in computed tomography images that prevent detection of endoleaks during follow-ups [8]. In addition, numerous coils are often required, increasing costs and prolonging procedures. Amplatzer vascular plugs provide an alternative to coils [9], but are often too small to adequately occlude the voluminous false lumen. In cases where an extra-large embolic device is needed, stent grafts have been modified to act as vascular plugs [10]. However, choosing the correct size of stent is difficult, as the false lumen will shrink as it depressurises [11]. Liquid embolic agents, such as Onyx ${ }^{\circledR}$ liquid embolic system, have been used to improve the efficacy of other embolic devices [12], but have toxicity issues [13]. Complete false lumen embolisation has the potential to improve patient care, but is limited by complications with currently available devices and agents. Importantly, current liquid embolic agents were not specifically designed to fill false lumen volumes. As such, there is an unmet need for a disease-specific device for false lumen embolisation in TBAD.

The design of such a device is not trivial. An effective false lumen embolic agent must: (1) be capable of catheter-based delivery, (2) be biocompatible, (3) conform to the shape of the false lumen, (4) withstand constant changes in aortic geometry, (5) degrade at a rate compatible with 
that of FLT, (6) mimic the material properties of intraluminal thrombus, (7) be easy to use in the clinic, and (8) not be prohibitively expensive. Based on these design criteria, an injectable biomaterial capable of forming a cohesive embolus within the entire false lumen volume would provide the best solution. Ideally, the biomaterial would have mechanical properties that mimicked a natural human thrombus to be compatible with the fragile, elastic tissue of a dissected aorta. There are no such commercially available devices or agents that satisfy these design criteria. Recently, Avery et al. [14] reported a novel shear-thinning biomaterial composed of gelatin and silicate nanoparticles that satisfies many of the above criteria. This biomaterial was used to successfully occlude blood flow in vitro and in vivo. However, evidence of the long-term stability of the material is lacking and it is unknown if the material would be suitable in the application of TBAD.

We hypothesise that materials with higher total solid concentrations will have the most stable mechanical properties and be most suitable for TBAD embolisation. However, to be clinically relevant the material must also be injectable through long catheters. In the case of TBAD, catheters are typically $1 \mathrm{~m}$ long and can range in diameter depending on the patient (e.g. 24F catheter requires a vessel diameter of $8 \mathrm{~mm}$ to access the aorta [5]). Furthermore, silk fibroin has been shown to modify rheological properties of some hydrogels [15] and could be a useful additive in the application of TBAD false lumen occlusion. Therefore, the objective of this study was to investigate blends of gelatin, silicate nanoparticles and silk fibroin to create a range of shear-thinning biomaterials for use as an embolic agent in proof-of-concept TBAD false lumen occlusion experiments. 


\section{MATERIALS AND METHODS}

\subsection{Materials and preparation}

\section{Materials}

Gelatin (type A, porcine skin, 300 bloom, G1890) and phosphate buffered saline (PBS; pH 7.4) were purchased from Sigma Aldrich (St. Louis, MO, USA). Synthetic silicate nanoparticles (Laponite ${ }^{\circledR}$ RD) were supplied by Talas Ltd. (Brooklyn, NY, USA) and Bombyx mori Silk fibroin powder was purchased from New Directions (Sydney, NSW, Australia) and used as received. Ultra-purified, Type 1 water was produced using a Milli-Q water purification system (Millipore Corp., Burlington, MA, USA).

\section{Preparation of composite materials}

Sample preparation methods were as previously described [14], with minor modifications to accommodate higher viscosities and the inclusion of silk fibroin. Stocks of $12 \%(\mathrm{w} / \mathrm{v})$ gelatin, $12 \%(\mathrm{w} / \mathrm{v})$ silk fibroin, and $8-12 \%(\mathrm{w} / \mathrm{v})$ silicate nanoparticles were prepared in water, then combined to give composites of desired proportions, diluting with water where necessary. Gelatin was dissolved by stirring for $1 \mathrm{~h}$ in $60^{\circ} \mathrm{C}$ water. Silicate nanoparticles were dispersed by stirring in $4^{\circ} \mathrm{C}$ water to slow gelation until an opaque gel formed, then left at room temperature until transparent. Silk fibroin was dispersed by stirring in water at $60^{\circ} \mathrm{C}$ to aid mixing upon combining stock solutions. Stock solutions were combined by repeatedly alternating between vigorous mixing using an electric drill with stirring attachment (Drill Mixer; Uni-Pro, Kilsyth, VIC, Australia) and heating in a $60^{\circ} \mathrm{C}$ water bath until a homogenous mixture was produced. Composite materials were stored at $4^{\circ} \mathrm{C}$ and used within $24 \mathrm{~h}$.

A total of 24 different composite materials were investigated (Table 1) with total solid concentrations of $8 \%, 10 \%$, and $12 \%(\mathrm{w} / \mathrm{v})$. Silk fibroin formed $0 \%, 5 \%, 10 \%$ or $20 \%(\mathrm{w} / \mathrm{w})$ of the total solids. The remainder of the total solids was divided between silicate nanoparticles and gelatin in ratios of 75:25 or 50:50 (silicate nanoparticles:gelatin). The sample naming convention has also been adapted from Avery et al. [14]. Composite materials are named according to the total solid concentration (w/v), the percentage of silk fibroin within the solid material, and the ratio of silicate nanoparticles to gelatin (e.g. composite material 10CM5-75 contains $10 \%(\mathrm{w} / \mathrm{v})$ solid matter of which $5 \%(\mathrm{w} / \mathrm{w})$ is silk fibroin and $75 \%(\mathrm{w} / \mathrm{w})$ is silicate nanoparticles). 
Table 1: Composition (wt. \%) of each composite material.

\begin{tabular}{|c|c|c|c|c|}
\hline Sample name & 8CM0-75 & 8CM5-75 & 8CM10-75 & 8CM20-75 \\
\hline Water & 92 & 92 & 92 & 92 \\
\hline Silk & 0 & 0.4 & 0.8 & 1.6 \\
\hline Gelatin & 2 & 1.9 & 1.8 & 1.6 \\
\hline Nanoparticles & 6 & 5.7 & 5.4 & 4.8 \\
\hline Sample name & 10CM0-75 & 10CM5-75 & 10CM10-75 & 10CM20-75 \\
\hline Water & 90 & 90 & 90 & 90 \\
\hline Silk & 0 & 0.5 & 1 & 2 \\
\hline Gelatin & 2.5 & 2.375 & 2.25 & 2 \\
\hline Nanoparticles & 7.5 & 7.125 & 6.75 & 6 \\
\hline Sample name & 12CM0-75 & 12CM5-75 & $12 \mathrm{CM} 10-75$ & 12CM20-75 \\
\hline Water & 88 & 88 & 88 & 88 \\
\hline Silk & 0 & 0.6 & 1.2 & 2.4 \\
\hline Gelatin & 3 & 2.85 & 2.7 & 2.4 \\
\hline Nanoparticles & 9 & 8.55 & 8.1 & 7.2 \\
\hline Sample name & 8CM0-50 & 8CM5-50 & 8 CM10-50 & 8CM20-50 \\
\hline Water & 92 & 92 & 92 & 92 \\
\hline Silk & 0 & 0.4 & 0.8 & 1.6 \\
\hline Gelatin & 4 & 3.8 & 3.6 & 3.2 \\
\hline Nanoparticles & 4 & 3.8 & 3.6 & 3.2 \\
\hline Sample name & 10CM0-50 & 10CM5-50 & 10CM10-50 & 10CM20-50 \\
\hline Water & 90 & 90 & 90 & 90 \\
\hline Silk & 0 & 0.5 & 1 & 2 \\
\hline Gelatin & 5 & 4.75 & 4.5 & 4 \\
\hline Nanoparticles & 5 & 4.75 & 4.5 & 4 \\
\hline Sample name & 12CM0-50 & 12CM5-50 & 12CM10-50 & 12CM20-50 \\
\hline Water & 88 & 88 & 88 & 88 \\
\hline Silk & 0 & 0.6 & 1.2 & 2.4 \\
\hline Gelatin & 6 & 5.7 & 5.4 & 4.8 \\
\hline Nanoparticles & 6 & 5.7 & 5.4 & 4.8 \\
\hline
\end{tabular}




\subsection{Compression testing}

\section{Sample preparation}

Freshly prepared material was poured into $10 \mathrm{~mm}$ thick moulds and stored at $4^{\circ} \mathrm{C}$ for $24 \mathrm{~h}$. Cylindrical samples were cut using a $10 \mathrm{~mm}$ diameter sharp cutting dye to create samples with a height:diameter ratio of 1 .

\section{Compression tests}

Compression tests were performed on a custom uniaxial testing machine [16-18] equipped with a $10 \mathrm{~N}$ load cell at a $0.1 \mathrm{~mm} / \mathrm{s}$ displacement rate. Each sample was subjected to 10 loading/unloading pre-conditioning cycles to $10 \%$ strain to ensure they were in a repeatable reference state [19], then tested to $70 \%$ strain. Stress-strain curves were generated using the force and displacement data collected from each test. Stress $(\sigma)$ was calculated by: $\sigma=\mathrm{F} / \mathrm{A}$, where $\mathrm{F}$ is the force $(\mathrm{N})$ applied on the sample, and $\mathrm{A}$ is the initial cross-sectional area $\left(\mathrm{m}^{2}\right)$ of the sample. Strain $(\epsilon)$ was calculated by: $\epsilon=\Delta \mathrm{l} / \mathrm{l}_{0}$, where $\Delta \mathrm{l}$ is the displacement of the compression platen and $l_{0}$ is the initial height of the sample. The compressive modulus $(E)$ was derived from the gradient of stress-strain curves within the initial linear region between 0 and 0.1 strain.

\section{Stability of compressive behaviour}

The mechanical stability of composite materials was studied by compression testing samples over several weeks. Following previous work [17, 18], samples were fully immersed in PBS and incubated at $37^{\circ} \mathrm{C}$ until tested. Testing was performed on days $0,1,3,5,7,9$ and every three days thereafter until day 21, without replacing samples. PBS was changed every third day. Day 0 testing was performed on un-hydrated samples to mimic the state of the material prior to injection. All subsequent tests were performed on hydrated samples, though the samples were not immersed in any solution during testing. Additionally, samples were tested at ambient room temperature as it was not possible to perform testing at $37^{\circ} \mathrm{C}$.

\subsection{Injectability}

\section{Preparation}

Injection tests were performed using disposable $20 \mathrm{ml}$ luer-lock syringes (barrel diameter: 19.6 $\mathrm{mm}$; Terumo Corporation, Shibuya, Japan) loaded with $20 \mathrm{ml}$ of composite material. Imitation catheters were fashioned from $1.0 \mathrm{~m}$ of clear, flexible polyvinyl chloride (PVC) tubing (PVC 
Tubes Online, Essex, England) and connected to syringes by luer-to-barb fittings (Cole-palmer, Vernon Hills, IL, USA).

To load syringes for testing, a syringe was back-filled with an excess of composite material, capped, then centrifuged (Allegra X-12R, Beckman Coulter, Pasadena, CA, USA) without a plunger at $3000 \mathrm{rpm}$ for $3 \mathrm{~min}$ to separate air from the material. Next, a plunger was inserted and $20 \mathrm{ml}$ of material was injected into a second syringe with depressed plunger via a luer-toluer connector (Cole-palmer, Vernon Hills, IL, USA). Care was taken to avoid introducing air pockets. Loaded syringes were stored upright at $4{ }^{\circ} \mathrm{C}$ for $24 \mathrm{~h}$ to density separate any remaining air, then allowed to come to room temperature prior to testing.

\section{Injection force testing}

To get an estimate of injection force limits, we began by injecting the biomaterial blend through a catheter. When the catheter became blocked, that is, the manual pressure applied could no longer extrude the material, we marked the point when extrusion ceased. We then used an Instron 5982 Universal Testing System (Instron, Melbourne, VIC, Australia) with a $500 \mathrm{~N}$ load cell in compressive mode to measure the force needed to inject material to that point. We deemed $\sim 175 \mathrm{~N}$ to be the upper injection force limit. Composite materials capable of being manually injected were selected for further injectability testing. The force required to inject each material was then measured for each combination of material and catheter.

Prepared syringes were positioned below the upper compression platen and held upright by a purpose-made apparatus. The upper compression platen depressed the syringe plunger at a speed set to give an injection rate of $3 \mathrm{ml} / \mathrm{min}$. The force on the syringe plunger was recorded using Bluehill version 3 software. The injection force was taken as the average force from the transition from strictly linear increase to the plateau region of the curve.

\section{Effect of catheter diameter}

A range of catheter internal diameters (IDs) were tested to investigate what effect it may have on the injection force. Catheter IDs of $2.5 \mathrm{~mm}, 3.0 \mathrm{~mm}, 3.5 \mathrm{~mm}$, and $4.0 \mathrm{~mm}$ were chosen based on clinical reasoning. Given a standard $24 \mathrm{~F}$ delivery device requires a vessel ID of 8.0 $\mathrm{mm}$ to access the aorta [5], a maximum catheter outer diameter (OD) of $5.0 \mathrm{~mm}$ was decided (equivalent to a 15 French catheter). A catheter with $5.0 \mathrm{~mm}$ OD could reasonably have an ID of $4.0 \mathrm{~mm}$, providing the upper limit of this study. Diameters were confirmed with digital callipers. 


\subsection{In vitro degradation rates}

Degradation testing was performed as per Avery et al. [15], using PBS in place of blood plasma. Composite material $(1 \mathrm{ml})$ was loaded into $2 \mathrm{ml}$ Eppendorf tubes (VWR, Radnor, PA, USA) and centrifuged (Allegra X-12R; Beckman Coulter, Pasadena, CA, USA) at $2000 \mathrm{rpm}$ for 1 min using a swinging bucket rotor to form a flat interface. Samples were weighed on a fourdigit balance before adding $1 \mathrm{ml}$ PBS then incubated at $37^{\circ} \mathrm{C}$. Every $48 \mathrm{hrs}$ for 30 days, PBS was removed, the samples re-weighed, and fresh PBS replaced. Degradation was calculated by: $\mathrm{D} \%=\left[\left(\mathrm{W}_{\mathrm{s}}-\mathrm{W}_{0}\right) / \mathrm{W}_{0}\right] \times 100$, where $\mathrm{D} \%$ is the percentage change in weight, $\mathrm{W}_{\mathrm{s}}$ is the weight of material measured at each time point, and $\mathrm{W}_{0}$ is the initial wet weight of the material.

\subsection{Experimental TBAD Model}

\section{Geometry}

Two different realistic TBAD geometries were created for this study. To begin with, we created an anatomically-correct 3D aorta, supra-aortic branching vessels and iliac arteries in computeraided design using available dimensions [20] (Figure 1A). We then used two patient-specific false lumens from our patient database; one of which had a single entry tear and single exit back into the true lumen (Figure 1B), whereas the other had an additional communicating channel approximately midway along the septum of the true and false lumens (Figure 1C). Geometries (in .stl format) were merged using Meshmixer (Autodesk, Inc.) and 3D printed in multiple parts with polylactic acid (PLA) using an Ultimaker $2+(0.8 \mathrm{~mm}$ nozzle; $0.15 \mathrm{~mm}$ layer height; $20 \%$ infill). As we needed to visualise the inside of the printed false lumen geometry during the experiments, we split the false lumen in half and replaced one half with a Perspex cover.

\section{Flow Loop Experiments}

The experimental flow loop is shown in Figure 2A with TBAD geometries shown in Figures $2 \mathrm{~B}$ and $2 \mathrm{C}$. The experimental set-up consisted of a pulsatile pump connected to a series tubes, pressure gauges, control valves, reservoir and the experimental TBAD model itself. Jig clamps and sealant were necessary to ensure the Perspex cover was water-tight. In this proof-ofconcept study we used water as the blood analogue and tested three flow rates; low $=3.8$, medium $=8.3$ and high $=11.4 \mathrm{~L} / \mathrm{min}$. 

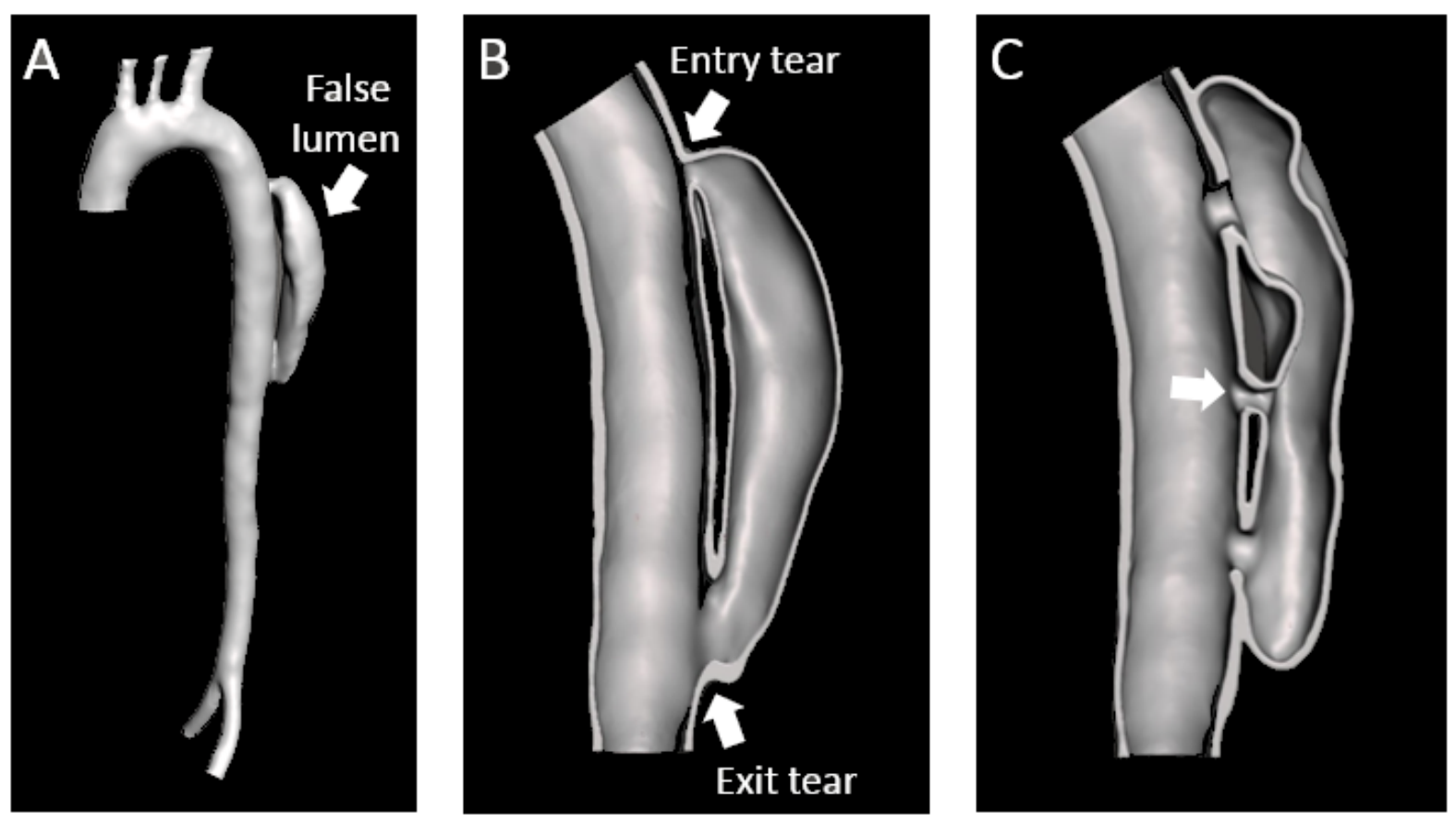

Figure 1: (A) Full 3D TBAD geometry of aorta, supra-aortic branching vessels, dissected region (true and false lumen) and iliac arteries. (B) Geometry \#1 with a single entry and exit tear. (C) Geometry \#2 with single entry and exit tears, and one communicating channel (arrow).

The biomaterial was prepared (as described earlier) in batches prior to experiments being carried out and stored at $4^{\circ} \mathrm{C}$. Red food dye was used to colour the biomaterial and help visualisation during experiments. Material was separated into $25 \mathrm{~mL}$ syringes and centrifuged at $3000 \mathrm{rpm}$ for 2 minutes to remove air pockets. The injection tubing was inserted through the 3-way injection device, through the iliac artery into the TBAD model, guided into the desired injection location and fixed in place using a small amount of adhesive. A photo was taken of the injection tubing placement to enable subsequent analysis of the injection angle after completion of the experiment. We initially pumped fluid slowly through the setup to remove air bubbles. Once desired flow settings were reached, the biomaterial was injected into the false lumen model. The total injection time was recorded until full flow occlusion in the injection tear was observed, with initial and final syringe volumes also recorded calculate the total volume of injected biomaterial. Once occluded, the flow was maintained for an additional 5 min. For a single experiment, the pulsatile flow was maintained for $60 \mathrm{~min}$. The visualisation of experimental results and subsequent analysis was conducted in RStudio (Boston, MA). 
A total of 78 experiments were performed (using a single biomaterial) under 18 different test conditions to account for the two different geometries, three flow rates, and differing number of tears in each geometry.

\subsection{Statistical analysis}

All analyses were performed using GraphPad Prism 7 version 7.0d for Mac OS X (GraphPad Software Inc., La Jolla, CA, USA). Data are expressed as mean \pm standard deviation (SD). The Shapiro-Wilks test was used to confirm the normal distribution of data. Data was analysed by one-way analysis of variance with Holm-Sidak's multiple comparisons test for $>2$ group data sets, and unpaired Student's t-test for 2-group data sets. All biomaterial samples were tested in triplicate. Significance was determined by $P$-values $<0.05$. Data was not blinded. 

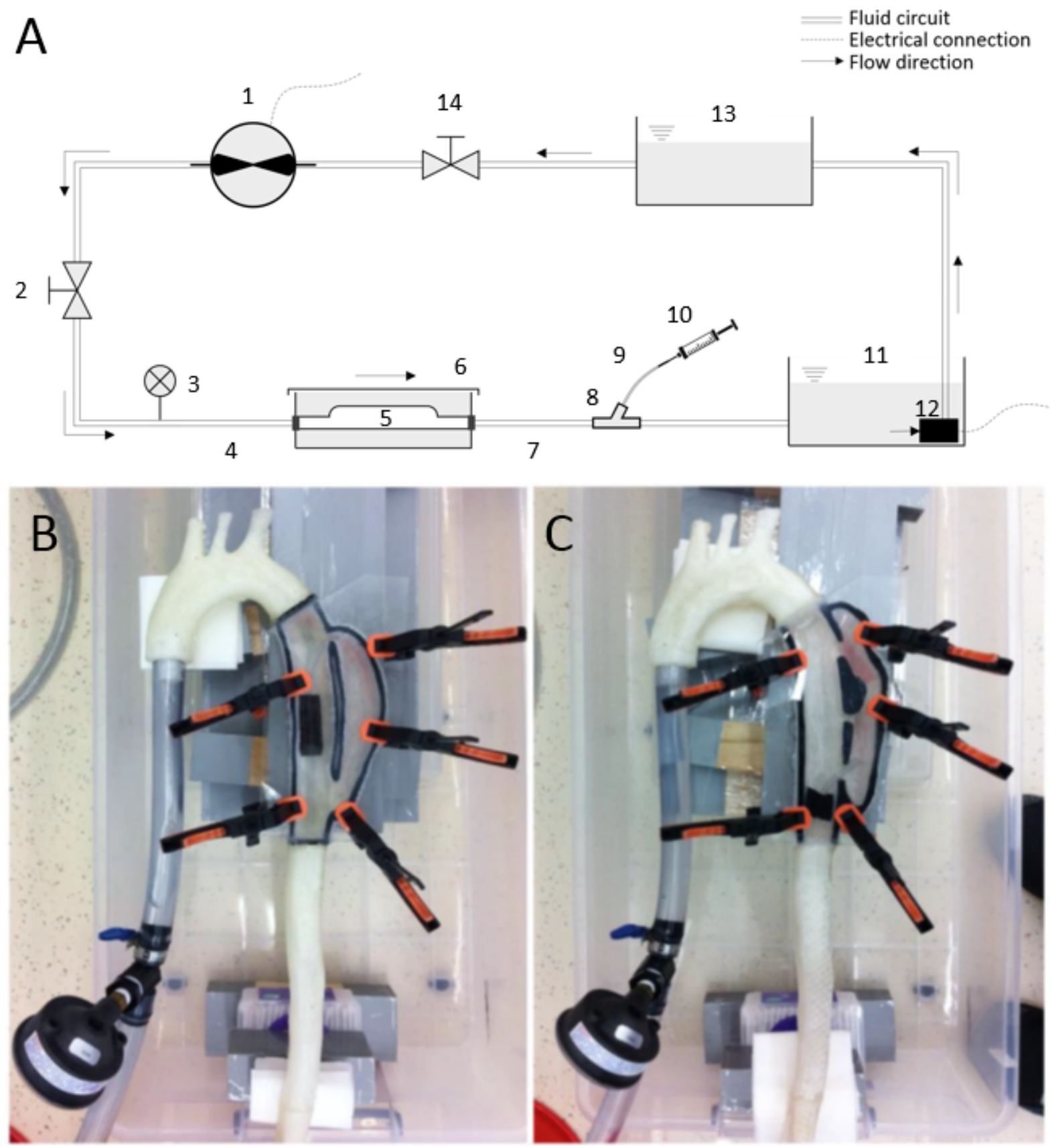

Figure 2: (A) Schematic of experimental flow loop. 1: pulsatile cardiac pump, 2: resistance valve, 3: pressure gauge, 4: $19 \mathrm{~mm}$ PVC piping, 5: aortic dissection model, 6: model support, 7: $13 \mathrm{~mm}$ PVC piping, 8: 3-way injection device, 9: $4 \mathrm{~mm}$ injection catheter, 10: $25 \mathrm{ml}$ syringe with biomaterial, 11: water reservoir \#1, 12: water pump, 13: water reservoir \#2, 14: flow control valve. (B) TBAD geometry \#1 with two tears. (C) TBAD geometry \#2 with three tears. 


\section{RESULTS}

\subsection{Compressive behaviour}

The change in compressive modulus for each material over the 30-day study period is presented in Figure 3. In general, increasing total solid concentration resulted in increased compressive moduli. Exposure to physiological conditions had a dramatic effect on compressive modulus that was determined by the ratio of silicate nanoparticles to gelatin. The compressive moduli of materials with 50:50 ratio was higher on Day 0 compared to materials with a 75:25 ratio, but declined significantly when exposed to physiological conditions (Table 2), stabilising below $10 \mathrm{kPa}$ by Day 5 . In contrast, the moduli of materials with a 75:25 ratio increased significantly (Table 2), stabilising by approximately Day 7. Due to the apparent stability of materials from Day 7 onwards, compressive moduli for individual materials between Day 7 and Day 21 were averaged to obtain a 'stable' compressive modulus (Table 3). Stable compressive moduli ranged from $2.9 \pm 0.7 \mathrm{kPa}$ to $5.5 \pm 1.2 \mathrm{kPa}$ for materials with a 50:50 ratio, and $35.1 \pm 8.9 \mathrm{kPa}$ to $117.6 \pm 15.2 \mathrm{kPa}$ for materials with a 75:25 ratio. The effect of silk fibroin was more pronounced amongst materials with a 75:25 ratio, as all materials containing silk fibroin demonstrated a significantly lower stable compressive modulus compared to their respective controls (Table 3). Whilst this trend was also present in materials with a 50:50 ratio, it was not consistently significant (Table 3 ).

The change in compressive modulus observed for materials with different ratios of gelatin to silicate nanoparticles is also reflected in the stress-strain curves. Stress-strain curves for materials with a 75:25 ratio were characteristic of elastomers on Day 0, but became viscoelastic after exposure to $\mathrm{PBS}$ at $37^{\circ} \mathrm{C}$ with apparent elastic behaviour in the strain range up to $10 \%$ deformation (Figure 4A(i)) [21]. Materials with a 50:50 ratio were viscoelastic on Day 0 with elastic behaviour up to $\sim 45 \%$ deformation, but become soft elastomers after Day 1 (Figure 4A(ii)). Stress-strain curves produced after Day 0 show a large degree of overlap, indicating materials remained stable over a wide range of deformation during the study period (stressstrain curves for all materials are available in the Supplementary Data section, Figures S2-S25). Materials with higher silicate nanoparticle content also maintained their cylindrical shape for the duration of the study period, with no visible loss in structural integrity (Figure 4B(i), unlike materials with low silicate nanoparticle content. (Figure 4B(ii)).

Due to the very low compressive moduli of materials with 50:50 ratio of silicate nanoparticles to gelatin, they were eliminated from consideration and are not discussed further. 

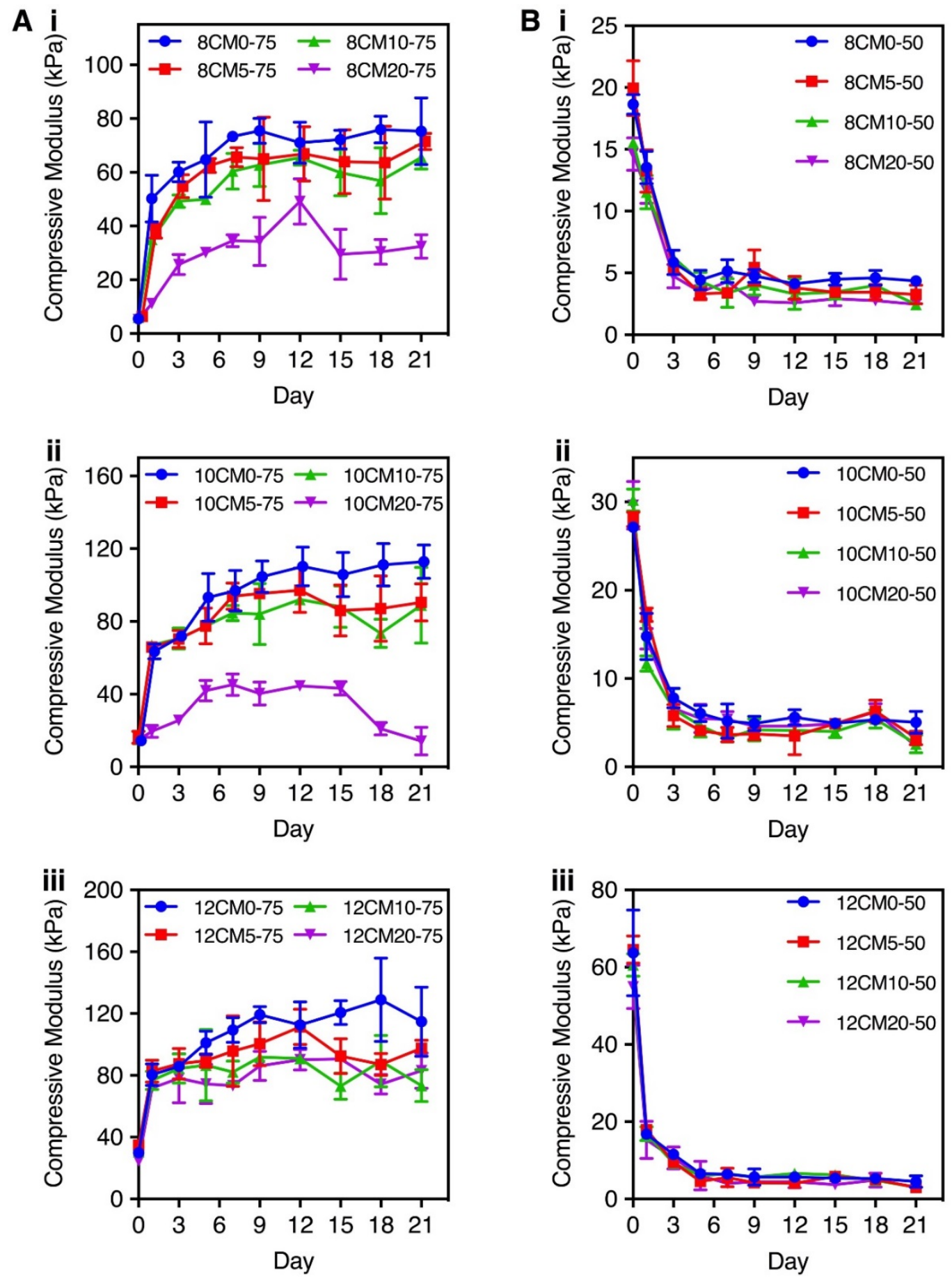

Figure 3: Change in compressive modulus over time. (A) materials with silicate nanoparticle-gelatin ratio of 75:25 and total solid concentration of (i) $8 \%$, (ii) $10 \%$, and (iii) 12\%; and, (B) materials with silicate nanoparticle-gelatin ratio of 50:50 and total solid concentration of (i) $8 \%$, (ii) $10 \%$, and (iii) $12 \%$. Materials were incubated in PBS at $37^{\circ} \mathrm{C}$ after Day 0. Data are mean \pm SD $(n=3)$. 
Table 2: Overview table of unpaired Students t-tests comparing the compressive modulus for each material on Day 0 and Day 1.

\begin{tabular}{|c|c|c|c|c|c|}
\hline \multicolumn{3}{|c|}{ Difference } & \multicolumn{3}{|c|}{ Difference } \\
\hline Material & $\begin{array}{l}\text { between means } \\
\qquad(\mathrm{kPa})\end{array}$ & P-value & Material & $\begin{array}{c}\text { between means } \\
\qquad(\mathrm{kPa})\end{array}$ & P-value \\
\hline $8 \mathrm{CM} 0-50$ & $-5.1 \pm 0.9$ & $<0.01$ & 8CM0-75 & $44.8 \pm 5.1$ & $<0.001$ \\
\hline 8CM5-50 & $-6.7 \pm 1.6$ & $<0.05$ & 8CM5-75 & $31.7 \pm 1.6$ & $<0.0001$ \\
\hline $8 \mathrm{CM} 10-50$ & $-4.0 \pm 0.8$ & $<0.01$ & 8CM10-75 & $29.0 \pm 0.8$ & $<0.0001$ \\
\hline $8 \mathrm{CM} 20-50$ & $-3.0 \pm 1.0$ & $<0.05$ & $8 \mathrm{CM} 20-75$ & $6.0 \pm 1.4$ & $<0.05$ \\
\hline 10CM0-50 & $-12.3 \pm 1.6$ & $<0.01$ & 10CM0-75 & $49.1 \pm 2.5$ & $<0.0001$ \\
\hline 10CM5-50 & $-10.8 \pm 0.6$ & $<0.0001$ & 10CM5-75 & $49.5 \pm 2.3$ & $<0.0001$ \\
\hline 10CM10-50 & $-18.5 \pm 0.9$ & $<0.0001$ & 10CM10-75 & $51.8 \pm 1.5$ & $<0.0001$ \\
\hline 10CM20-50 & $-15.1 \pm 1.7$ & $<0.001$ & 10CM20-75 & $4.6 \pm 2.1$ & 0.09 \\
\hline $12 \mathrm{CM} 0-50$ & $-46.5 \pm 6.8$ & $<0.01$ & 12CM0-75 & $50.5 \pm 4.2$ & $<0.001$ \\
\hline 12CM5-50 & -52.4 to -40.8 & $<0.0001$ & 12CM5-75 & $47.9 \pm 4.3$ & $<0.001$ \\
\hline 12CM10-50 & $-43.8 \pm 2.0$ & $<0.0001$ & 12CM10-75 & $46.2 \pm 3.4$ & $<0.001$ \\
\hline 12CM20-50 & $-39.2 \pm 4.2$ & $<0.001$ & 12CM20-75 & $47.2 \pm 2.4$ & $<0.0001$ \\
\hline
\end{tabular}


Table 3: Overview table of stable compressive moduli. The compressive modulus was derived by averaging measurements between Day 7 and Day 21 for each material. Data are mean \pm SD $(\mathrm{n}=18)$. Materials were compared to their respective controls (in bold) by one-way analysis of variance with Holm-Sidak's multiple comparisons tests $\left({ }^{*} P<0.05,{ }^{* *} P<0.01,{ }^{* * *} P<0.001\right.$, ${ }^{* * * *}$ $P<0.0001)$.

\begin{tabular}{|c|c|c|c|}
\hline Material & $\begin{array}{c}\text { Stable } \\
\text { compressive } \\
\text { modulus }(\mathrm{kPa})\end{array}$ & Material & $\begin{array}{l}\text { Stable } \\
\text { compressive } \\
\text { modulus }(\mathrm{kPa})\end{array}$ \\
\hline 8CM0-50 & $4.6 \pm 0.6$ & 8CM0-75 & $73.8 \pm 6.0$ \\
\hline $8 \mathrm{CM} 5-50$ & $3.8 \pm 1.0^{* *}$ & 8CM5-75 & $66.1 \pm 9.4^{* *}$ \\
\hline 8CM10-50 & $3.4 \pm 0.9 * * *$ & $8 \mathrm{CM} 10-75$ & $61.8 \pm 7.3^{* * * *}$ \\
\hline 8CM20-50 & $2.9 \pm 0.7 * * * *$ & $8 \mathrm{CM} 20-75$ & $35.1 \pm 8.9 * * * *$ \\
\hline 10CM0-50 & $5.2 \pm 0.9$ & 10СМ0-75 & $106.9 \pm 10.5$ \\
\hline 10CM5-50 & $4.2 \pm 1.5^{*}$ & 10CM5-75 & $91.7 \pm 10.8 * * *$ \\
\hline 10CM10-50 & $4.0 \pm 1.1^{*}$ & 10CM10-75 & $85.2 \pm 12.1 * * * *$ \\
\hline 10CM20-50 & $4.7 \pm 1.3$ & 10CM20-75 & $34.8 \pm 13.5^{* * * *}$ \\
\hline 12CM0-50 & $5.5 \pm 1.2$ & 12CM0-75 & $117.6 \pm 15.2$ \\
\hline 12CM5-50 & $4.6 \pm 1.4$ & 12CM5-75 & $97.4 \pm 13.7 * * * *$ \\
\hline 12CM10-50 & $5.4 \pm 1.4$ & $12 \mathrm{CM} 10-75$ & $83.4 \pm 11.2 * * * *$ \\
\hline 12CM20-50 & $4.1 \pm 1.1 * *$ & 12CM20-75 & $82.9 \pm 9.4 * * * *$ \\
\hline
\end{tabular}



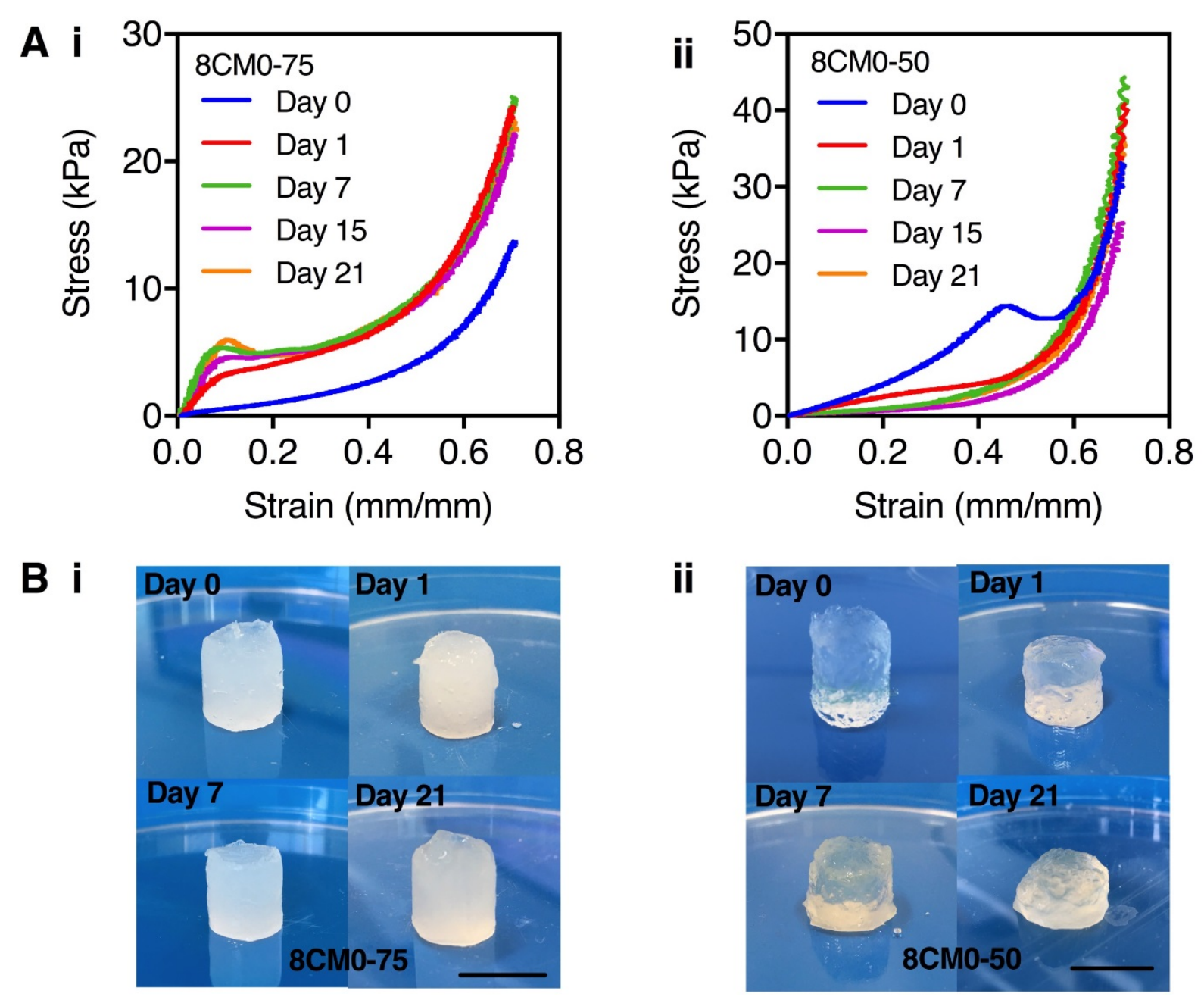

Figure 4: (A) Representative stress-strain curves of (i) 8CM0-75 and (ii) 8CM0-50 at different time points. (B) Images of (i) 8CM0-75 and (ii) 8CM0-50 compression test samples taken on days $0,1,7$, and 21 . Scale bars represent $10 \mathrm{~mm}$.

\subsection{Injectability}

Injectability was improved by lowering total solid concentrations (Supplementary Data, Table S1), with materials containing $8 \%(\mathrm{w} / \mathrm{v})$ solids being injectable through all catheter sizes. Materials containing $10 \%(\mathrm{w} / \mathrm{v})$ solids were injectable only through catheters with IDs of 3.5 $\mathrm{mm}$ and $4.0 \mathrm{~mm}$, whereas materials with $12 \%(\mathrm{w} / \mathrm{v})$ solids were not manually injectable through any of the catheters tested.

The injection forces for each material are presented in Figure 5. Injection forces were a function of total solid concentration, silk fibroin content and catheter diameter and ranged from a 
minimum of $64.8 \pm 3.1 \mathrm{~N}$ for $8 \mathrm{CM} 20-75$ when injected through a $4.0 \mathrm{~mm}$ catheter to a maximum of $157.5 \pm 1.2 \mathrm{~N}$ for $8 \mathrm{CM} 0-75$ when injected through a $2.5 \mathrm{~mm}$ catheter.

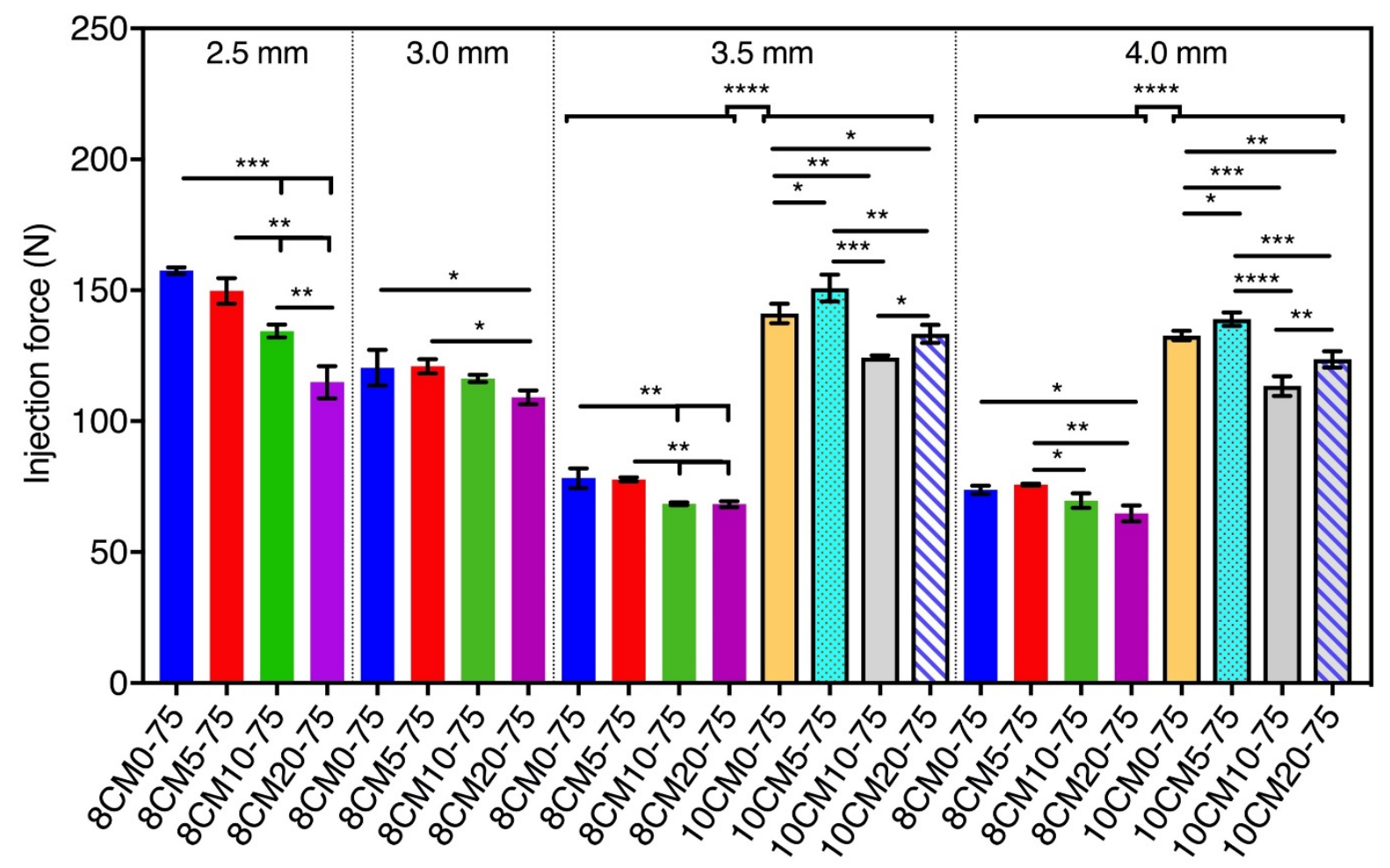

Figure 5: The injection force of materials capable of manual injection through catheters with internal diameters of $2.5 \mathrm{~mm}, 3.0 \mathrm{~mm}, 3.5 \mathrm{~mm}$, and $4.0 \mathrm{~mm}$. $P$-values were determined by one-way analysis of variance with Holm-Sidak's multiple comparisons tests $\left({ }^{*} P<0.05\right.$, $\left.{ }^{* *} P<0.01,{ }^{* * *} P<0.001,{ }^{* * * *} P<0.0001\right)$. Data are mean $\pm \mathrm{SD}(\mathrm{n}=3)$.

When materials were injected through catheters with wider IDs, significantly lower forces were required, however $8 \%(\mathrm{w} / \mathrm{v})$ materials required similar force when injected through $3.5 \mathrm{~mm}$ and $4.0 \mathrm{~mm}$ catheters (Supplementary Data, Figure S26). For a given catheter diameter, higher total solid concentrations were associated with higher injections forces. For example, the force required to inject 10CM0-75 through a $3.5 \mathrm{~mm}$ catheter was significantly higher than $8 \mathrm{CM} 0$ 75 when injected through the same catheter $(141.1 \pm 3.7 \mathrm{~N}$ versus $78.2 \pm 3.8 \mathrm{~N}, P<0.0001)$. When $8 \%(\mathrm{w} / \mathrm{v})$ materials were compared, there was a general trend of increasing silk fibroin content resulting in lower injection forces, though materials with $0 \%$ and $5 \%$ silk fibroin required similar force when injected through all catheters. When $10 \%(\mathrm{w} / \mathrm{v})$ materials were compared, silk fibroin produced no clear effect in a given catheter, yet the same pattern of injection forces is repeated in both $3.5 \mathrm{~mm}$ and $4.0 \mathrm{~mm}$ catheters. 
For each material, the injection force increased linearly before plateauing as material began to extrude from the catheter (injection force profiles for all materials are available in the Supplementary Data section, Figures S27-S32). Compared to materials without silk fibroin, those containing silk fibroin showed a more curved transition to the plateau region (Figures 6).
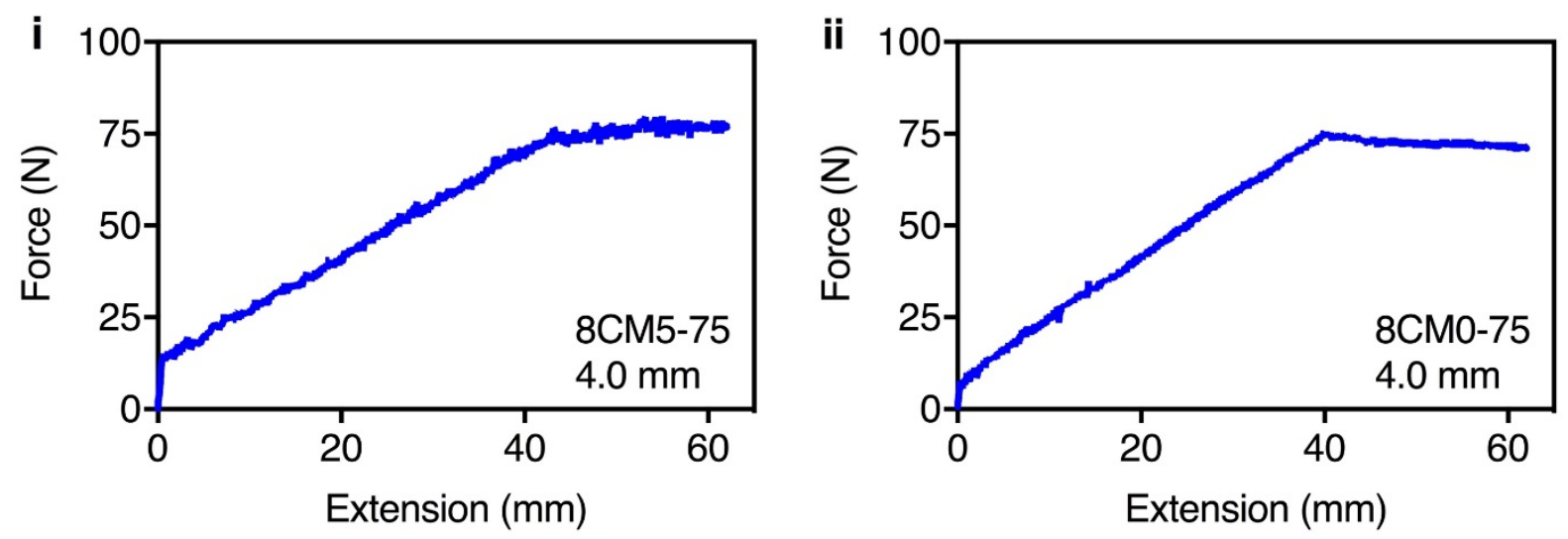

Figure 6: Representative injection force profiles of (i) 8CM5-75 and (ii) 8CM0-75 when injected through a $4.0 \mathrm{~mm}$ catheter. The addition of silk fibroin created a smoother transition to the plateau region.

\subsection{Degradation}

The change in weight for each material is presented in Figure 7. After 30 days, the total change in weight across all materials ranged from $-1.5 \pm 0.3 \%$ for $12 \mathrm{CM} 0-75$ to $-8.4 \pm 0.5 \%$ for 8CM20-75. Some materials initially increased in weight, though weight decreased in a strongly linear manner for all materials over the time period. Other factors being constant, materials with lower total solid concentration or higher silk fibroin content degraded faster, though degradation rates were only significantly quicker for materials containing $20 \%$ silk fibroin (Table 4). 

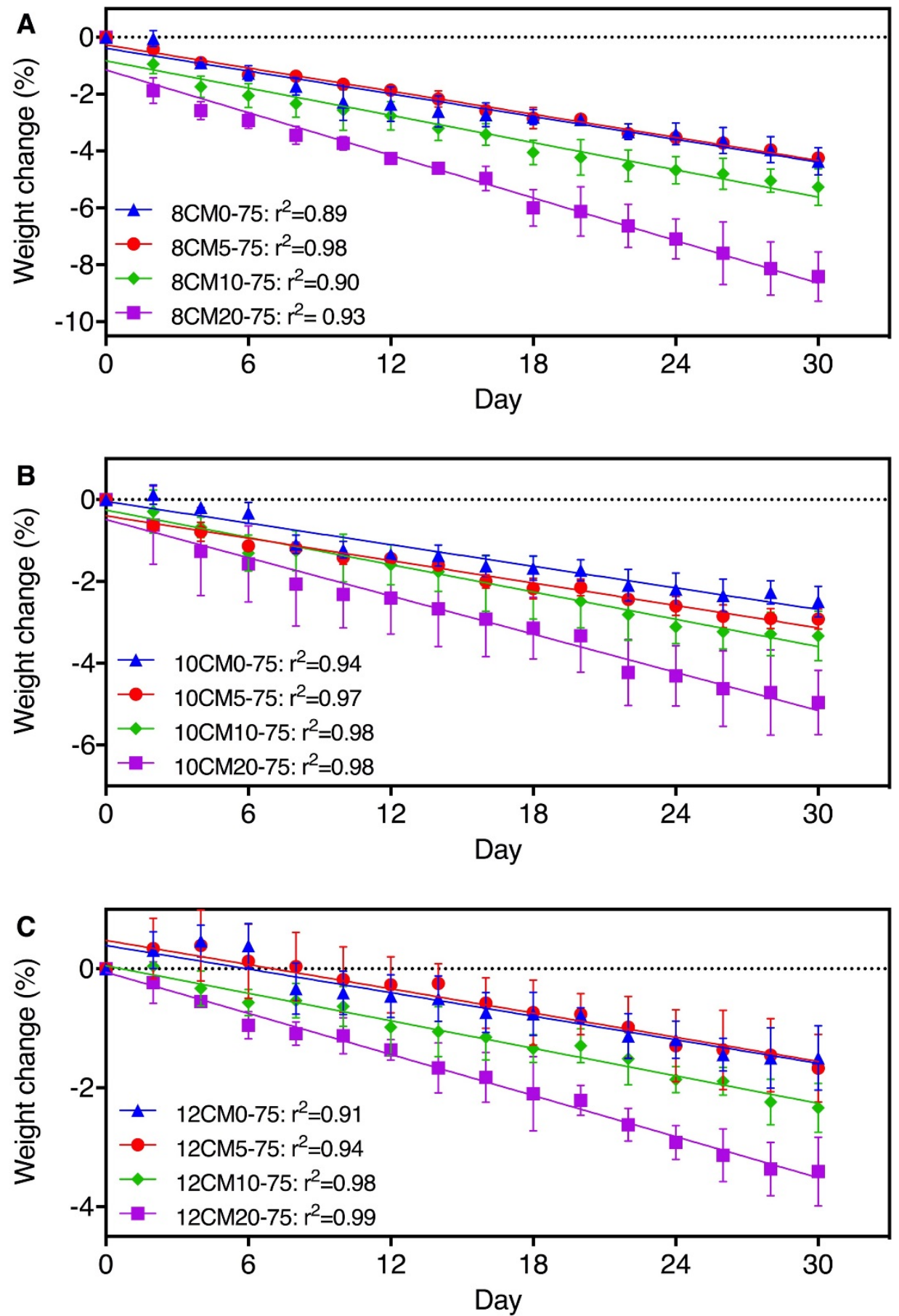

Figure 7: The change in weight of materials with 75:25 silicate nanoparticles to gelatin and total solid concentrations (w/v) of (A) $8 \%$, (B) $10 \%$, and (C) $12 \%$ stored in PBS at $37^{\circ} \mathrm{C}$ over 30 days. Data are mean $\pm S D(n=3)$. 
Table 4: Comparisons between degradation rates. Gradients obtained from linear regression performed on weight change data were compared within each total solid concentration by oneway analysis of variance with Holm-Sidak's multiple comparisons tests, $\mathrm{n}=3$. Bold $P$-values are significant.

\begin{tabular}{lccc}
\hline Material & $8 \mathrm{CM} 5-75$ & $8 \mathrm{CM} 10-75$ & $8 \mathrm{CM} 20-75$ \\
\hline $8 \mathrm{CM} 0-75$ & 0.8 & 0.2 & $<\mathbf{0 . 0 1}$ \\
$8 \mathrm{CM} 5-75$ & - & 0.2 & $<\mathbf{0 . 0 1}$ \\
$8 \mathrm{CM} 10-75$ & - & - & $<\mathbf{0 . 0 1}$ \\
\hline Material & $10 \mathrm{CM} 5-75$ & $10 \mathrm{CM} 10-75$ & $10 \mathrm{CM} 20-75$ \\
\hline 10CM0-75 & 0.7 & 0.1 & $<\mathbf{0 . 0 1}$ \\
10CM5-75 & - & 0.1 & $<\mathbf{0 . 0 1}$ \\
10CM10-75 & - & - & $\mathbf{0 . 0 2}$ \\
\hline Material & $12 \mathrm{CM} 5-75$ & $12 \mathrm{CM} 10-75$ & $12 \mathrm{CM} 20-75$ \\
\hline 12CM0-75 & 0.8 & 0.3 & $<\mathbf{0 . 0 1}$ \\
12CM5-75 & - & 0.3 & $<\mathbf{0 . 0 1}$ \\
$12 \mathrm{CM} 10-75$ & - & - & $\mathbf{0 . 0 1}$ \\
\hline
\end{tabular}

\subsection{Application in TBAD}

Flow occlusion into false lumen

For these experiments we used the biomaterial blend 8CM5-75 and found that it was able to occlude flow into the false lumen in 77/78 experiments (99\%). Figure 8 shows a visual summary of some success cases. These preliminary experiments show that although the injection tear is always occluded, not all tears in the dissection are occluded when injected via a single tear (e.g. Geometry \#2, proximal tear, high flow rate). This can be remedied by determining the volume of the false lumen beforehand using CTA-based 3D reconstructions and using the appropriate amount of biomaterial to ensure complete occlusion. However, even with incomplete filling of the false lumen, when we maintained pulsatile flow for prolonged 
period of time (Figure 9), the flow into the false lumen remained occluded with no signs of recanalisation. We could not determine any association between experimental factors and the lack of success in the single failed experiment (Figure S33).
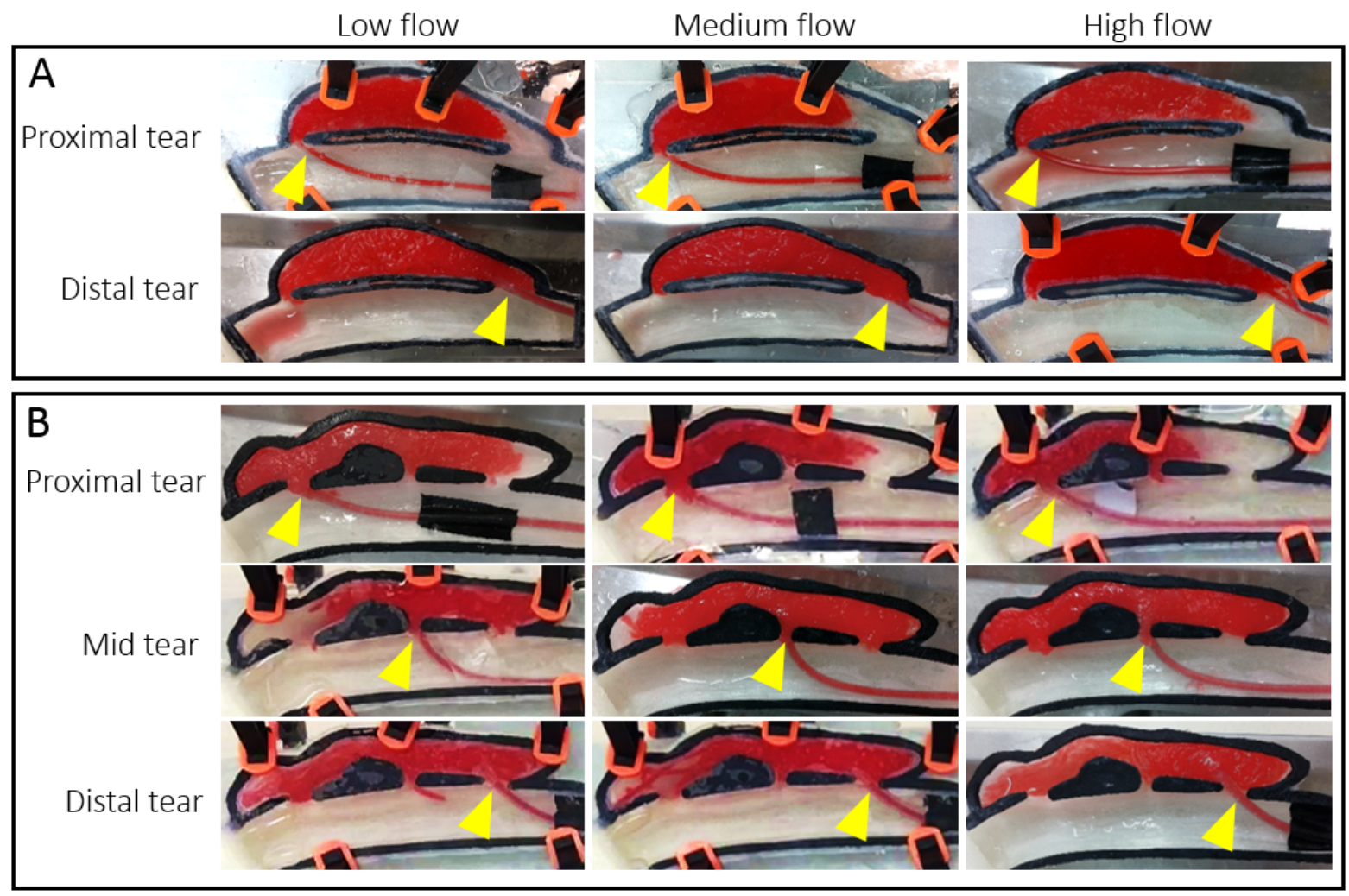

Figure 8: Biomaterial occluding flow into the false lumen for TBAD geometry \#1 (A) and geometry \#2 (B) at low, medium and high flow rates. Experiments shown for proximal, mid and distal injection of biomaterial into false lumen. Yellow arrows indicate the injection catheter. 


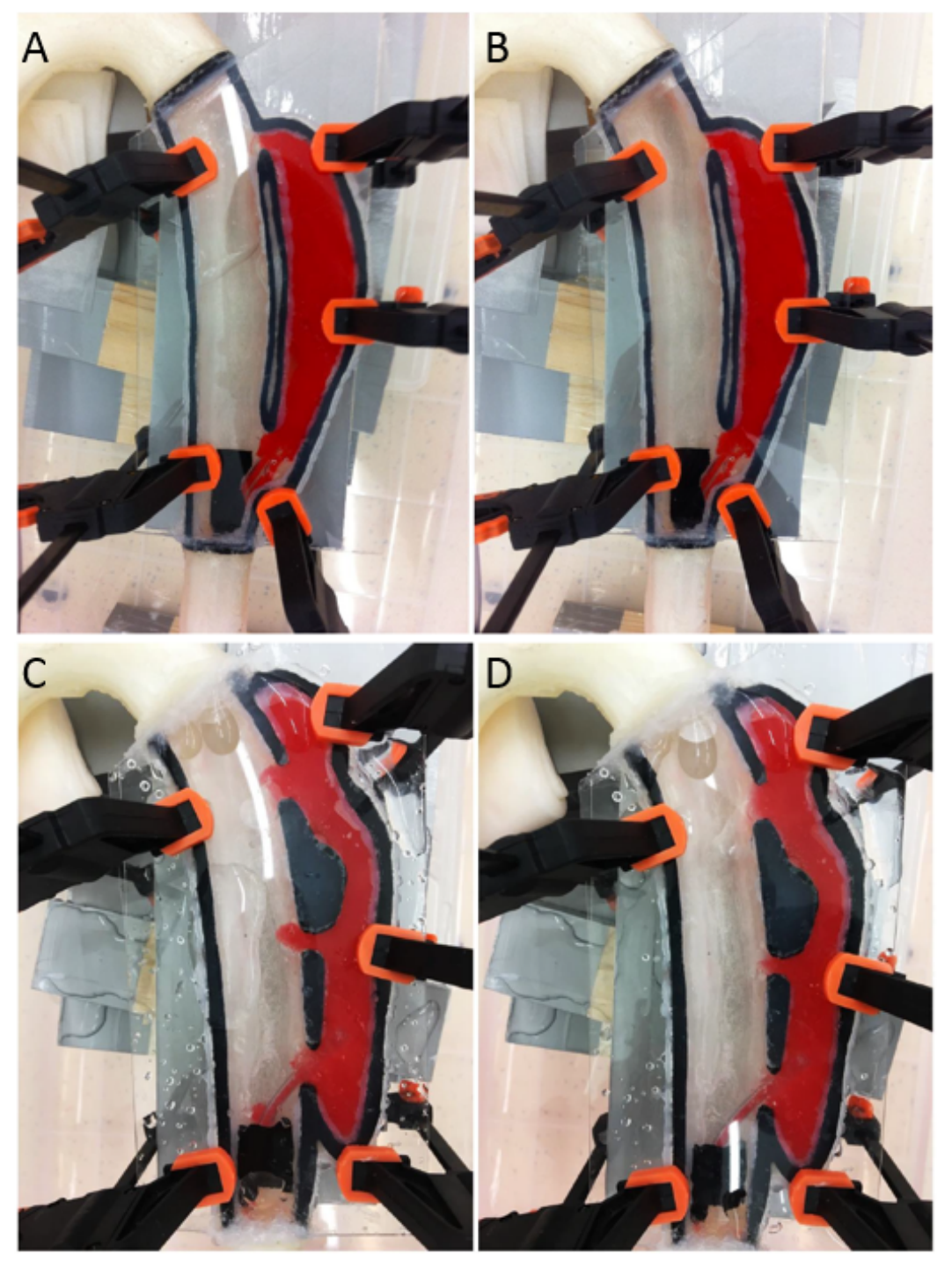

Figure 9: Biomaterial occluding flow into the false lumen for TBAD geometry $\# 1$ at (A) $t=0$ $\mathrm{s}$ and $(\mathrm{B}) \mathrm{t}=5 \mathrm{~min}$ during medium flow $(8.3 \mathrm{~mL} / \mathrm{min})$ conditions. Occlusion for TBAD geometry \#2 at (C) t $=0 \mathrm{~s}$ and (D) $\mathrm{t}=60 \mathrm{~min}$ during low flow $(3.8 \mathrm{~mL} / \mathrm{min})$ conditions. Both cases show distal tear injection conditions.

\section{Injected biomaterial volumes}

We determined the average injection volume to range between $25-45 \mathrm{~mL}$, as shown in Figure 10. There were no significant differences in injection volume for all combinations of flow and injection location, when compared between the same geometry. This implies that injection volumes required for occlusion are not dependent on cardiac output or injection method. We noted some material loss, especially in Geometry \#1, during injection. However, there was no correlation between injection rate, injection angle and injection angle with biomaterial loss. 

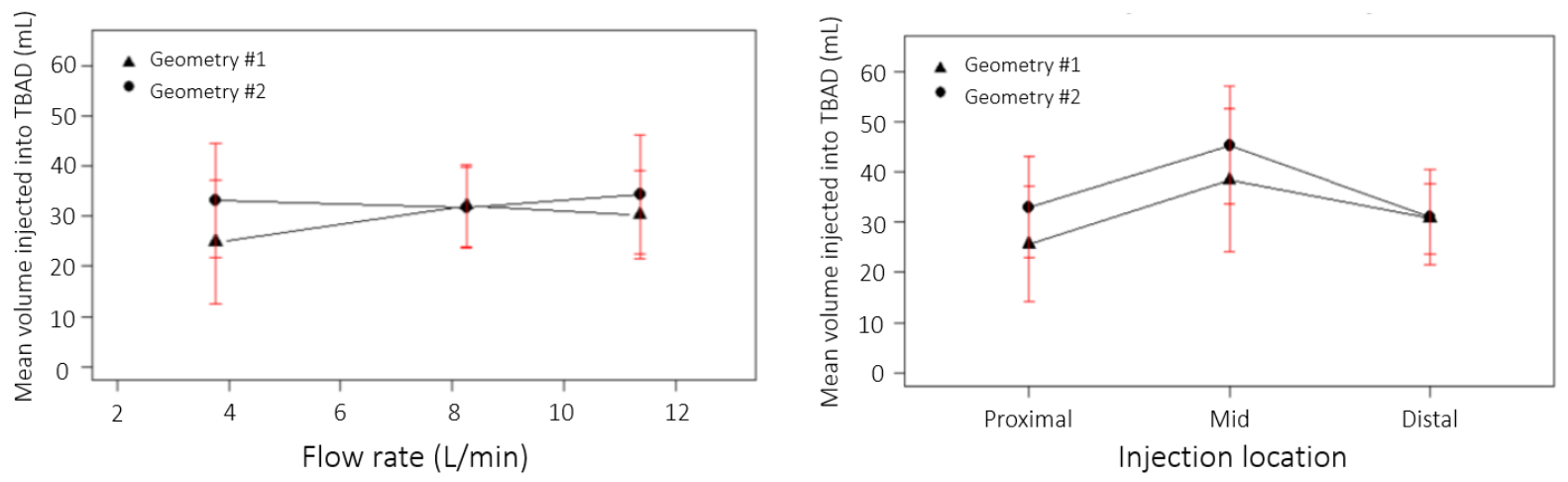

Figure 10: Average injected biomaterial volumes required to occlude flow in both TBAD geometries for each flow rate (left) and each injection location (right). 


\section{DISCUSSION}

The aim of this study was to develop and test a shear-thinning biomaterial for application as a minimally invasive embolic agent in TBAD. We used blends of gelatin, silicate nanoparticles and silk fibroin as our biomaterial and have shown the compressive behaviour, injectability and degradation rates are a function of the total solid concentration, the silk fibroin content and the ratio of silicate nanoparticles to gelatin. Based on our results, we propose that $8 \mathrm{CM} 0-75$, 8CM5-75 and 8CM10-75 warrant further investigation as biomaterials for false lumen embolisation. We then performed proof-of-concept studies using anatomically-correct TBAD geometries in an experimental pulsatile flow-loop using the 8CM5-75 biomaterial. The biomaterial successfully occluded flow into the false lumen $99 \%$ experiments under a range of different operating conditions.

\subsection{Compressive behaviour}

To minimise material mismatch between an implanted biomaterial and the deformable tissue surrounding it, we aimed to design the false lumen embolic agent to mimic the mechanical properties of false lumen thrombus. However, to the best of our knowledge, the mechanical properties of a false lumen thrombus have not been investigated. Therefore, we assumed the stiffness closely resembles thrombi from abdominal aortic aneurysms, which have reported moduli of $50-200 \mathrm{kPa}$ ([22], $42-68 \mathrm{kPa}[23], 54 \mathrm{kPa}$ [24], and $\sim 13-59 \mathrm{kPa}$ [25]. The moduli obtained for materials with a 75:25 silicate nanoparticle to gelatin ratio fall within these ranges.

The stark contrast in compressive behaviour between materials with 75:25 and 50:50 ratios of silicate nanoparticles to gelatin can be explained by the nature of the cross-linking between components. Gelatin forms electrostatic interactions with silicate nanoparticles in an optimum stoichiometric binding ratio of 3:1 (silicate nanoparticles:gelatin) [26], but can also form physical cross-links with other gelatin molecules at low temperature [27]. Cross-linking between gelatin molecules occurs at higher densities than between gelatin and silicate nanoparticles [28]. Furthermore, the stiffness of materials is determined by the density of crosslinks [29]. Hence, the compressive moduli of materials with a 50:50 ratio is higher on Day 0 compared to materials with a 75:25 ratio, potentially due to an excess of gelatin that creates a denser network of cross-links. This has previously been shown for other gelatin-silicate nanoparticle materials of up to $4 \%(\mathrm{w} / \mathrm{v})$ [30] and we have found this trend continues for higher concentrations. However, gelatin begins to liquefy at temperatures above $29^{\circ} \mathrm{C}$ [31], hence at $37^{\circ} \mathrm{C}$ the network of cross-linked gelatin dissociates, reducing the structural integrity. This is 
evident in the rapid decline in compressive modulus after exposure to physiological conditions and the change in the physical appearance of the samples, which appear to lose volume over time. In contrast, materials with a 75:25 ratio appear to be heat cured on exposure to physiological conditions. The profile of the stress-strain curves shows a large increase in stiffness following incubation at $37^{\circ} \mathrm{C}$ (Figure 1) suggesting the density of cross-links has increased. Low temperature $\left(<40^{\circ} \mathrm{C}\right)$ heat curing is able to improve the compressive behaviour of certain hydrogels, demonstrating this process is possible at physiological temperatures [32]. Previous work has also found the mechanical properties of composite materials with higher silicate nanoparticle to gelatin ratio to be enhanced by curing [30, 33].

Materials with high silicate nanoparticle content also demonstrate compatible deformation behaviour. Both the material and natural thrombi are viscoelastic, which is important as this property influences how pressure is distributed on the aortic wall [34]. Further, the elastic range of the material corresponds with the range of motion of the aorta, which can expand and contract by up to $10 \%$ during the cardiac cycle [35].

Multiple individual studies have demonstrated the stiffness of natural thrombi vary amongst patients $[22,23,25]$. The effect of altering the total concentration of solid components and the silk fibroin content suggests the mechanical properties of the material could be tuned to accommodate a range of thrombus moduli. However, the inverse relationship between silk fibroin content and compressive moduli suggests silk fibroin contributes poorly to the crosslinking density of the composite materials. This may be attributed to weak interactions between silk fibroin and the silicate nanoparticles [36]. Consequently, for a given total solid concentration, increasing the silk fibroin content weakens the material by lowering the gelatin and silicate nanoparticle content. Unless silk fibroin confers an in vivo benefit, a simpler approach to tuning the mechanical properties may be to eliminate silk fibroin and simply vary the total concentration of gelatin and silicate nanoparticles to achieve the desired stiffness. Additionally, the ability to tune the material stiffness is an advantage over current embolic agents.

\subsection{Injectability}

Intuitively, lower total solid concentrations and larger diameter catheters required lower injection forces. For $8 \%(\mathrm{w} / \mathrm{v})$ materials, the inverse relationship between silk fibroin content and injection force can be explained by a reduction in viscosity brought about by weak interactions between silk fibroin and silicate nanoparticles that potentially generate fewer 
cross-links [36]. However, this does not explain the increase in injection force observed for $10 \%(\mathrm{w} / \mathrm{v})$ materials containing silk fibroin. It is possible the trend in the $8 \%(\mathrm{w} / \mathrm{v})$ materials is erroneous, though it is more likely the results are due to changes in ambient temperature that occurred on the days $10 \%(\mathrm{w} / \mathrm{v})$ materials were tested. These temperature changes could not be controlled, but their effect could have been minimised had samples been randomised.

Injection forces greater than $100 \mathrm{~N}$ are generally regarded as inappropriate [37, 38]. As such, only $8 \%(\mathrm{w} / \mathrm{v})$ materials injected through a $4.0 \mathrm{~mm}$ catheter are clinically relevant. Avery et al. [14] recorded injection forces of $<30 \mathrm{~N}$ for $6 \%(\mathrm{w} / \mathrm{v})$ material injected through a $0.97 \mathrm{~mm}$ catheter using a $3 \mathrm{ml}$ syringe. As the radius of the catheter has more influence on flow than the viscosity of the material [39], it was expected the wider catheters in this study would compensate for the higher total solid concentrations. However, the larger $20 \mathrm{ml}$ syringe used here seems to have negated this entirely, as the minimum force recorded was $64.8 \pm 3.1 \mathrm{~N}$. We required a $20 \mathrm{ml}$ syringe considering the volume of the $4.0 \mathrm{~mm}$ catheter was $12.6 \mathrm{ml}$, making it impossible to measure the injection force using even a $10 \mathrm{ml}$ syringe. It is highly likely a smaller syringe diameter would improve injectability. Additionally, the injection force profiles provide some insight into how user-friendly the materials are. The curved transition from linear increase to the plateau region seen on the profiles of materials containing silk fibroin suggests more precise control and ease of use in comparison to those materials without silk. This could be an important aspect of the biomaterial if to be used clinically, as surgeons will require exact control over the delivery of the material into the false lumen.

\subsection{Degradation}

This study corroborates and extends the degradation test performed by Avery et al. [14] who found no or negligible degradation over $24 \mathrm{~h}$. Here we measured degradation over the 30-day study period and recorded changes ranging from $1.5-8.4 \%$ with faster degradation for materials with low total solid concentration or high silk fibroin content. The degradation rate is governed by the number of cross-links between components and the pore size [40, 41]. Higher silicate nanoparticle content produces smaller pores, slowing degradation [30], whilst silk fibroin interacts weakly with silicate nanoparticles [36], forming few cross-links, accelerating degradation. Hence, degradation rates can be tuned by altering the content of silicate nanoparticles and silk fibroin, as shown here.

The ability to tune the degradation rate by varying the composition implies it could be matched to the rate of false lumen thrombosis and aortic remodelling. Reports indicate complete false 
lumen thrombosis can occur within 3 months once blood flow through the false lumen is prevented $[42,43]$, however the rate of false lumen thrombosis has not been thoroughly investigated and it is possible the material will need to remain in place for longer.

Premature degradation leading to recanalization is an obvious concern. The strongly linear degradation rates suggest the length of time the material will remain implanted is highly predictable, though how well in vitro and in vivo degradation rates correlate is not known. The in vivo performance of the material has been tested successfully up to 24 days, however degradation was not quantified [14]. Silicate nanoparticles have shown prothrombotic activity by concentrating clotting factors and accelerating the formation of a blood clot on the surface of the material [44, 45], which could act as a barrier to hydrolytic and enzymatic breakdown, slowing in vivo degradation. However, the time frame and conditions used here do not allow extrapolations to be made about in vivo degradation. Ultimately, the minimal degradation observed validates a longer, more thorough investigation.

\subsection{Application in TBAD}

Our preliminary experiments show the biomaterial (8CM5-75) has potential in TBAD repair. Only one experiment (78 in total) failed to occlude flow and in most cases the false lumen was easily occluded and a range of injection locations and angles. We did notice that by injecting the biomaterial into only tear location, it was possible to not fully occlude the false lumen volume. However, in practice, the volume of the false lumen could be determined during the surgical planning stage from the 3D reconstructions and the correct amount of biomaterial used. Additionally, either a second catheter could be deployed so that two tear locations are tackled occluded during the surgery, or a single catheter cold be retracted before another catheter is inserted. Surgeons frequently gain aortic access via both femoral arteries and typically insert/retract devices minimally-invasively. Therefore in certain cases where a single catheter and pre-determined biomaterial injection volume is not adequate, the use of multiple catheters is not an obstacle to translation.

\subsection{Limitations}

First, compression testing was performed at room temperature, rather than $37^{\circ} \mathrm{C}$. Future testing should include pulsatile loading at $37^{\circ} \mathrm{C}$ to determine the possibility of in vivo fatigue failure. This temperature change may also be important for the injectability and clinical use; all experiments were performed at room temperature whereas a surgeon would be deploying the 
material into $37^{\circ} \mathrm{C}$ physiological environment. Second, the time frame investigated was too short to reliably predict the long-term behaviour of the material. It is also not known if degradation will continue linearly, or if it is compatible with the rate of aortic remodeling. However, Avery et al. show that their blend with only $6 \%$ solid matter was capable of occluding a porcine vein for at least 24 days [14]. Third, PBS was used in place of whole blood, which may have influenced degradation rates. A more comprehensive degradation study might use human blood at various haematocrit concentrations at $37^{\circ} \mathrm{C}$. Finally, the imitation catheters used in this study are not directly comparable to approved medical catheters and therefore the injection forces may be different. Ultimately, more in vitro and in vivo research is needed to determine if these biomaterials and this approach would be clinically useful in the treatment of TBAD.

\section{CONCLUSIONS}

We have identified shear-thinning biomaterials that have desirable compressive moduli, injectability and degradation rates for use as false lumen embolic agents in type B aortic dissection. The mechanical properties of these materials with a high silicate nanoparticle to gelatin ratio are compatible with those of natural aortic thrombus and could potentially be tuned to match individual patients. Materials with $8 \%(\mathrm{w} / \mathrm{v})$ total solid concentration required clinically relevant injection forces, although varying the injection parameters, such as syringe size, is a simple way to improve injectability. It is not known if degradation rates are compatible with aortic remodelling, however the minimal amount of degradation observed justifies continued investigation into these biomaterials and this application.

\section{ACKNOWLEDGEMENTS}

We would like to acknowledge funding from the National Health and Medical Research Council (Grants APP1063986 and APP1083572) and the Australian and New Zealand Society of Vascular Surgery (ANZSVS) Vascular Foundation. We are also are grateful to Lachlan Kelsey and Malcolm Stafford for technical assistance. 


\section{REFERENCES}

1. Howard, D.P.J., et al., Incidence, risk factors, outcome and projected future burden of acute aortic dissection. Annals of Cardiothoracic Surgery, 2014. 3(3): p. 278-284.

2. Nienaber, C.A. and R.E. Clough, Management of acute aortic dissection. The Lancet, 2015. 385(9970): p. 800-811.

3. Daily, P.O., et al., Management of acute aortic dissections. Annals of Thoracic Surgery, 1970. 10(3): p. 237-247.

4. Evangelista, A., et al., Insights from the International Registry of Acute Aortic Dissection. Circulation, 2018. 137(17): p. 1846.

5. Grabenwöger, M., et al., Thoracic Endovascular Aortic Repair (TEVAR) for the treatment of aortic diseases: A position statement from the European Association for Cardio-Thoracic Surgery (EACTS) and the European Society of Cardiology (ESC), in collaboration with the European Association of Percutaneous Cardiovascular Interventions (EAPCI). European Heart Journal, 2012. 33(13): p. 1558-1563.

6. Trimarchi, S., et al., Importance of false lumen thrombosis in type B aortic dissection prognosis. Journal of Thoracic and Cardiovascular Surgery, 2013. 145(3): p. S208S212.

7. Hussain, M.A., et al., Coil embolization of the false lumen in complicated type B aortic dissection. Annals of Vascular Surgery, 2015. 29(1): p. 125.e13-125.e17.

8. Prell, D., et al., Metal artifact reduction for clipping and coiling in interventional CArm CT. American Journal of Neuroradiology, 2010. 31(4): p. 634-639.

9. Mendes, B.C., et al., False lumen embolization to treat disseminated intravascular coagulation after thoracic endovascular aortic repair of type B aortic dissection. Journal of Endovascular Therapy, 2015. 22(6): p. 938-941.

10. Kölbel, T., et al., Distal false lumen occlusion in aortic dissection with a homemade extra-large vascular plug: the candy-plug technique. Journal of Endovascular Therapy, 2013. 20(4): p. 484-489.

11. Dake, M.D., et al., Endovascular stent-graft placement for the treatment of acute aortic dissection. New England Journal of Medicine, 1999. 340(20): p. 1546-1552.

12. Hofferberth, S.C., I.K. Nixon, and P.J. Mossop, Aortic false lumen thrombosis induction by embolotherapy (AFTER) following endovascular repair of aortic dissection. Journal of Endovascular Therapy, 2012. 19(4): p. 538-545.

13. Bakar, B., et al., Evaluation of the toxicity of onyx compared with n-butyl 2cyanoacrylate in the subarachnoid space of a rabbit model: An experimental research. Neuroradiology, 2010. 52(2): p. 125-134.

14. Avery, R.K., et al., An injectable shear-thinning biomaterial for endovascular embolization. Science Translational Medicine, 2016. 8(365): p. 365ra156.

15. $\mathrm{Wu}, \mathrm{J}$., et al., Rheological, mechanical and degradable properties of injectable chitosan/silk fibroin/hydroxyapatite/glycerophosphate hydrogels. Journal of the Mechanical Behavior of Biomedical Materials, 2016. 64: p. 161-172.

16. Macrae, R.A., K. Miller, and B.J.C.S.-R. Doyle, Methods in Mechanical Testing of Arterial Tissue: A Review. Strain, 2016. 52(5): p. 380-399.

17. Law, N., et al., Characterisation of hyaluronic acid methylcellulose hydrogels for $3 D$ bioprinting. Journal of the Mechanical Behavior of Biomedical Materials, 2018. 77: p. 389-399.

18. Giuseppe, M.D., et al., Mechanical behaviour of alginate-gelatin hydrogels for 3D bioprinting. Journal of the Mechanical Behavior of Biomedical Materials, 2018. 79: p. 150-157. 
19. Mullins, L., Softening of rubber by deformation. Rubber Chemistry and Technology, 1969. 42: p. 339-362.

20. Michaeu, A. Anatomy of the Aorta. Radiological classifications commonly used in medical imaging 2008 14/04/2018]; Available from: https://www.imaios.com/en/eCases/Channels/Radiology/Radiological-classifications-commonly-used-in-medicalimaging/Anatomy-of-the-aorta.

21. Alzoubi, M.F., S. Khateeb, and S. Al-Hallaj, Modeling of compression curves of phase change graphite composites using Maxwell and Kelvin models. Journal of Composite Materials, 2016. 50(8): p. 1123-1135.

22. Di Martino, E., et al., Biomechanics of abdominal aortic aneurysm in the presence of endoluminal thrombus: Experimental characterisation and structural static computational analysis. European Journal of Vascular and Endovascular Surgery, 1998. 15(4): p. 290-299.

23. Gasser, T.C., et al., Failure properties of intraluminal thrombus in abdominal aortic aneurysm under static and pulsating mechanical loads. Journal of Vascular Surgery, 2008. 48(1): p. 179-188.

24. Wang, D.H.J., et al., Mechanical properties and microstructure of intraluminal thrombus from abdominal aortic aneurysm. Journal of Biomechanical Engineering, 2001. 123(6): p. 536-539.

25. Hinnen, J.W., et al., Development of fibrinous thrombus analogue for in-vitro abdominal aortic aneurysm studies. Journal of Biomechanics, 2007. 40(2): p. 289-295.

26. Pawar, N. and H.B. Bohidar, Surface selective binding of nanoclay particles to polyampholyte protein chains. Journal of Chemical Physics, 2009. 131(4).

27. Hellio, D. and M. Djabourov, Physically and chemically crosslinked gelatin gels. Macromolecular Symposia, 2006. 241(1): p. 23-27.

28. Prado, J.R. and S. Vyazovkin, Melting of gelatin gels containing laponite, montmorillonite, and chitosan particles. Macromolecular Chemistry and Physics, 2014. 215(9): p. 867-872.

29. Lin, S. and L. Gu, Influence of crosslink density and stiffness on mechanical properties of type I collagen gel. Materials, 2015. 8(2): p. 551-560.

30. Li, C., et al., Gelatin effects on the physicochemical and hemocompatible properties of gelatin/PAAm/laponite nanocomposite hydrogels. ACS Applied Materials and Interfaces, 2015. 7(33): p. 18732-18741.

31. Djabourov, M., J. Leblond, and P. Papon, Gelation of aqueous gelatin solutions. I. Structural investigation. Journal de Physique, 1988. 49(2): p. 319-332.

32. Ferruti, P., M. Grigolini, and E. Ranucci, PHEMA hydrogels obtained by a novel lowheat curing procedure with a potential for in situ preparation. Macromolecular Bioscience, 2004. 4(6): p. 591-600.

33. Bhatnagar, D., et al., Hyaluronic acid and gelatin clay composite hydrogels: Substrates for cell adhesion and controlled drug delivery. Journal of Chemical and Biological Interfaces, 2014. 2: p. 1-11.

34. Hinnen, J.-W., et al., Effect of intraluminal thrombus on pressure transmission in the abdominal aortic aneurysm. Journal of Vascular Surgery, 2005. 42(6): p. 1176-1182.

35. Carrascosa, P., et al., Thoracic aorta cardiac-cycle related dynamic changes assessed with a 256-slice CT scanner. Cardiovascular Diagnosis and Therapy, 2013. 3(3): p. 125-128.

36. Dang, Q., et al., Silk fibroin/montmorillonite nanocomposites: Effect of $p H$ on the conformational transition and clay dispersion. Biomacromolecules, 2010. 11(7): p. 1796-1801. 
37. Khairoun, I., et al., Some factors controlling the injectability of calcium phosphate bone cements. Journal of Materials Science: Materials in Medicine, 1998. 9(8): p. 425-428.

38. Rungseevijitprapa, W. and R. Bodmeier, Injectability of biodegradable in situ forming microparticle systems (ISM). European Journal of Pharmaceutical Sciences, 2009. 36(4-5): p. 524-531.

39. Davidovits, P., Physics in biology and medicine. 3rd ed. 2008, San Diego: Academic Press.

40. Ozeki, M. and Y. Tabata, In vivo degradability of hydrogels prepared from different gelatins by various cross-linking methods. Journal of Biomaterials Science, Polymer Edition, 2005. 16(5): p. 549-561.

41. Kang, H.W., Y. Tabata, and Y. Ikada, Effect of porous structure on the degradation of freeze-dried gelatin hydrogels. Journal of Bioactive and Compatible Polymers, 1999. 14(4): p. 331-343.

42. Norberto, E.M.S., J. Taylor, and C. Vaquero, Aortic false lumen thrombosis and remodeling: The paradigm for endovascular repair of aortic dissection. Journal of Endovascular Therapy, 2012. 19(4): p. 546-548.

43. Norberto, E.M.S., et al., Coil embolization of persistent false lumen after stent graft repair of type B aortic dissection. Journal of Vascular Surgery, 2011. 54(1): p. 201204.

44. Gaharwar, A.K., et al., Shear-thinning nanocomposite hydrogels for the treatment of hemorrhage. ACS Nano, 2014. 8(10): p. 9833-9842.

45. Baker, S.E., et al., Controlling bioprocesses with inorganic surfaces: Layered clay hemostatic agents. Chemistry of Materials, 2007. 19(18): p. 4390-4392. 
SUPPLEMENTARY DATA

\title{
Development of a shear-thinning biomaterial as an endovascular embolic agent for the treatment of type $B$ aortic dissection
}

\author{
Matthew J. Moore ${ }^{1,2}$ Lauren Malaxos ${ }^{1,3}$ and Barry J. Doyle $\mathrm{e}^{1,3,4}$
}

1. Vascular Engineering Laboratory, Harry Perkins Institute of Medical Research, QEII Medical Centre, Nedlands and Centre for Medical Research, The University of Western Australia, Perth, Australia.

2. School of Biomedical Science, The University of Western Australia, Perth, Australia.

3. School of Engineering, The University of Western Australia, Perth, Australia.

4. Australian Research Council Centre for Personalised Therapeutics Technologies, Australia.

5. BHF Centre for Cardiovascular Science, The University of Edinburgh, Edinburgh, UK.

\section{Corresponding Author:}

Barry Doyle, Tel: +61 86151 1084, Fax:+61 86151 1084, Email: barry.doyle@uwa.edu.au 6 Verdun Street, Nedlands, Perth, WA 6009, Australia.

ORCID ID: 0000-0003-4923-2796 


\section{CONTENTS}

Figure S1: Anatomy of the aorta model used in the study.

\section{Stress-strain curves:}

Figure S2: 8CM0-75

Figure S2: 8CM5-75

Figure S4: 8CM10-75

Figure S5: 8CM20-75

Figure S6: 10CM0-75

Figure S7: 10CM5-75

Figure S8: 10CM10-75

Figure S9: 10CM20-75

Figure S10: 12CM0-75

Figure S11: 12CM5-75

Figure S12: 12CM10-75

Figure S13: 12CM20-75

Figure S14: 8CM0-50

Figure S15: 8CM5-50

Figure S16: 8CM10-50

Figure S17: 8CM20-50

Figure S18: 10CM0-50

Figure S19: 10CM5-50

Figure S20: 10CM10-50

Figure S21: 10CM20-50

Figure S22: 12CM0-75

Figure S23: 12CM5-50

Figure S24: 12CM10-50

Figure S25: 12CM20-50

\section{Injection force}

Figure S26: Injection forces

Figures S27: 8\% (w/v) materials injected through a $2.5 \mathrm{~mm}$ catheter Figures S28: 8\% (w/v) materials injected through a $3.0 \mathrm{~mm}$ catheter Figures S29: 8\% (w/v) materials injected through a $3.5 \mathrm{~mm}$ catheter Figures S30: $8 \%(\mathrm{w} / \mathrm{v})$ materials injected through a $4.0 \mathrm{~mm}$ catheter Figures S31: $10 \%(\mathrm{w} / \mathrm{v})$ materials injected through a $3.5 \mathrm{~mm}$ catheter Figures S32: 10\% (w/v) materials injected through a $4.0 \mathrm{~mm}$ catheter Table S1: Manual injectability

Figure S33: Occlusion experiments 

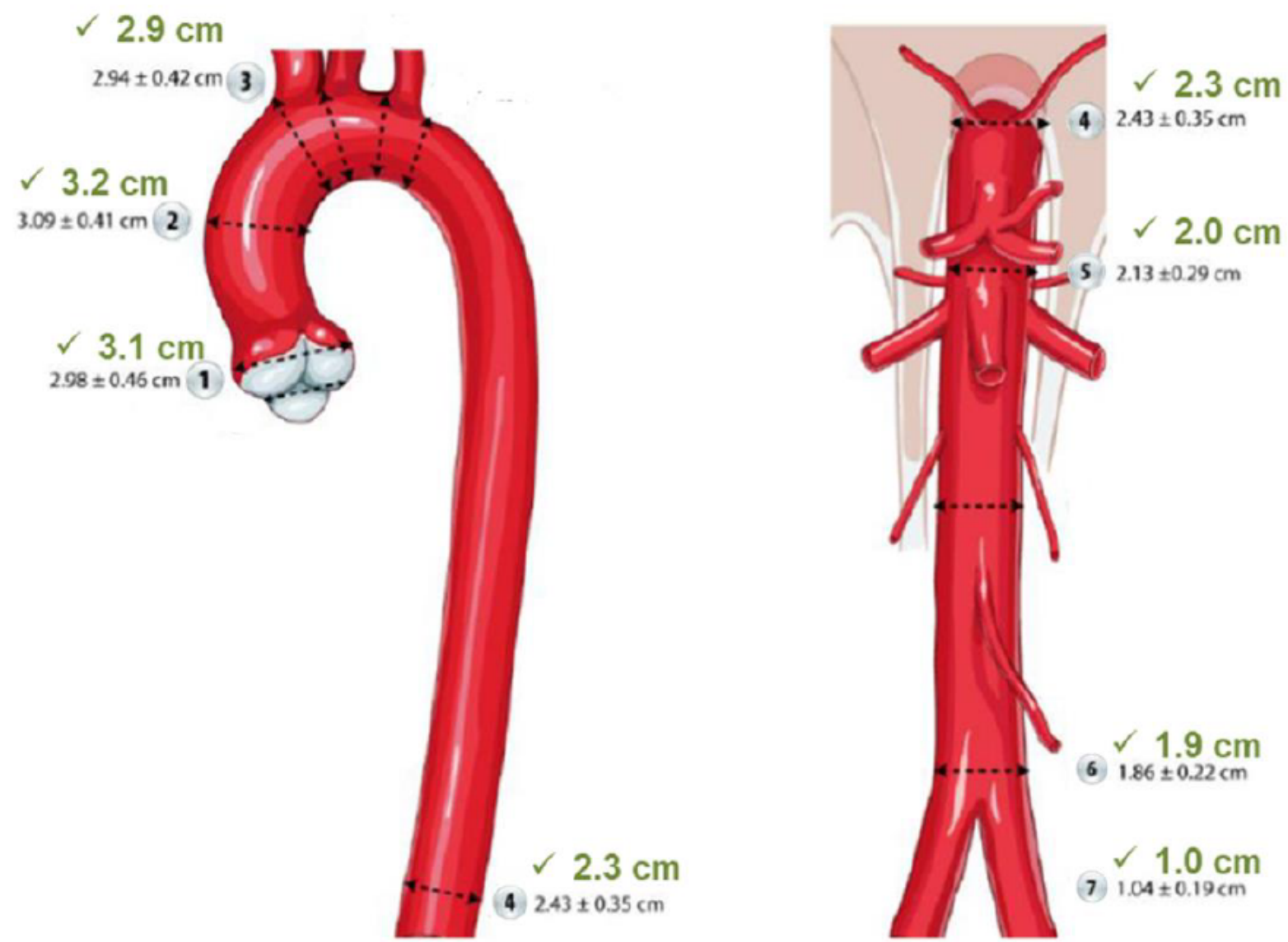

Figure S1. Summary of the aorta model created with final dimensions shown in green. Data was obtained from an online radiology training platform. 

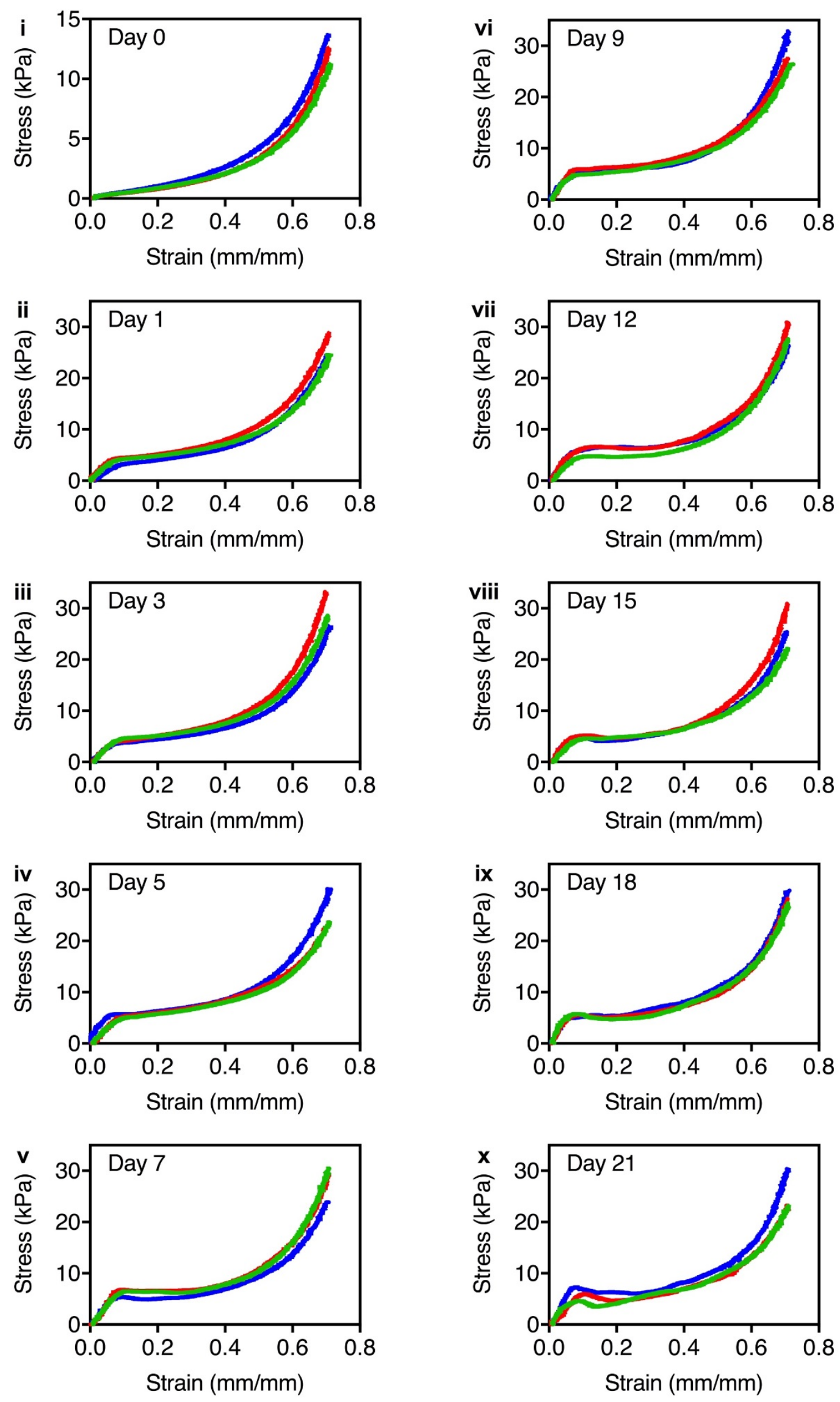

Figure S2. Stress-strain curves for 8CM0-75 produced on (i) day 0, (ii) day 1, (iii) day 3, (iv) day 5, (v) day 7, (vi) day 9, (vii) day 12, (viii) day 15, (ix) day 18, and (x) day 21. 

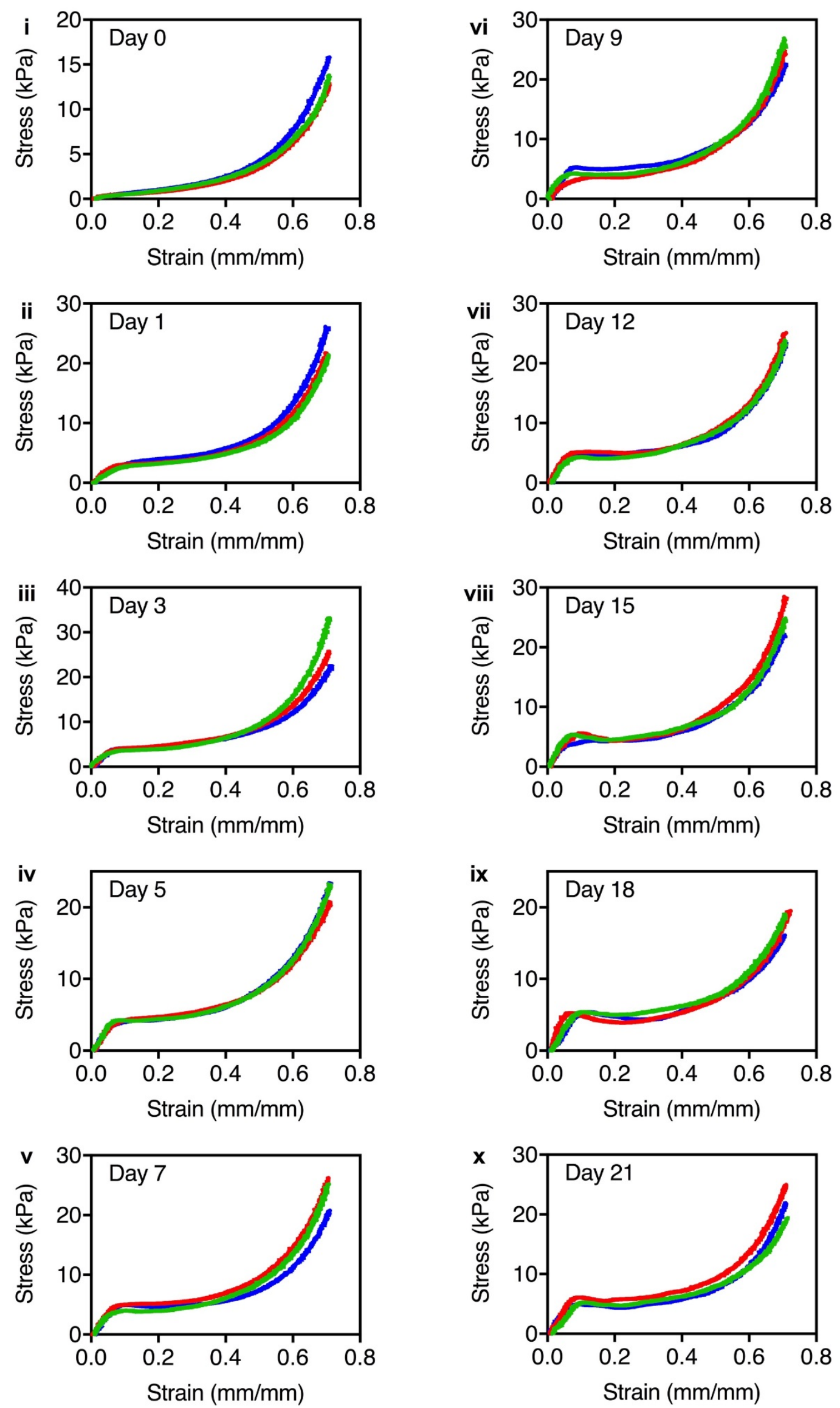

Figure S3. Stress-strain curves for 8CM5-75 produced on (i) day 0, (ii) day 1, (iii) day 3, (iv) day 5, (v) day 7, (vi) day 9, (vii) day 12, (viii) day 15, (ix) day 18, and (x) day 21. 

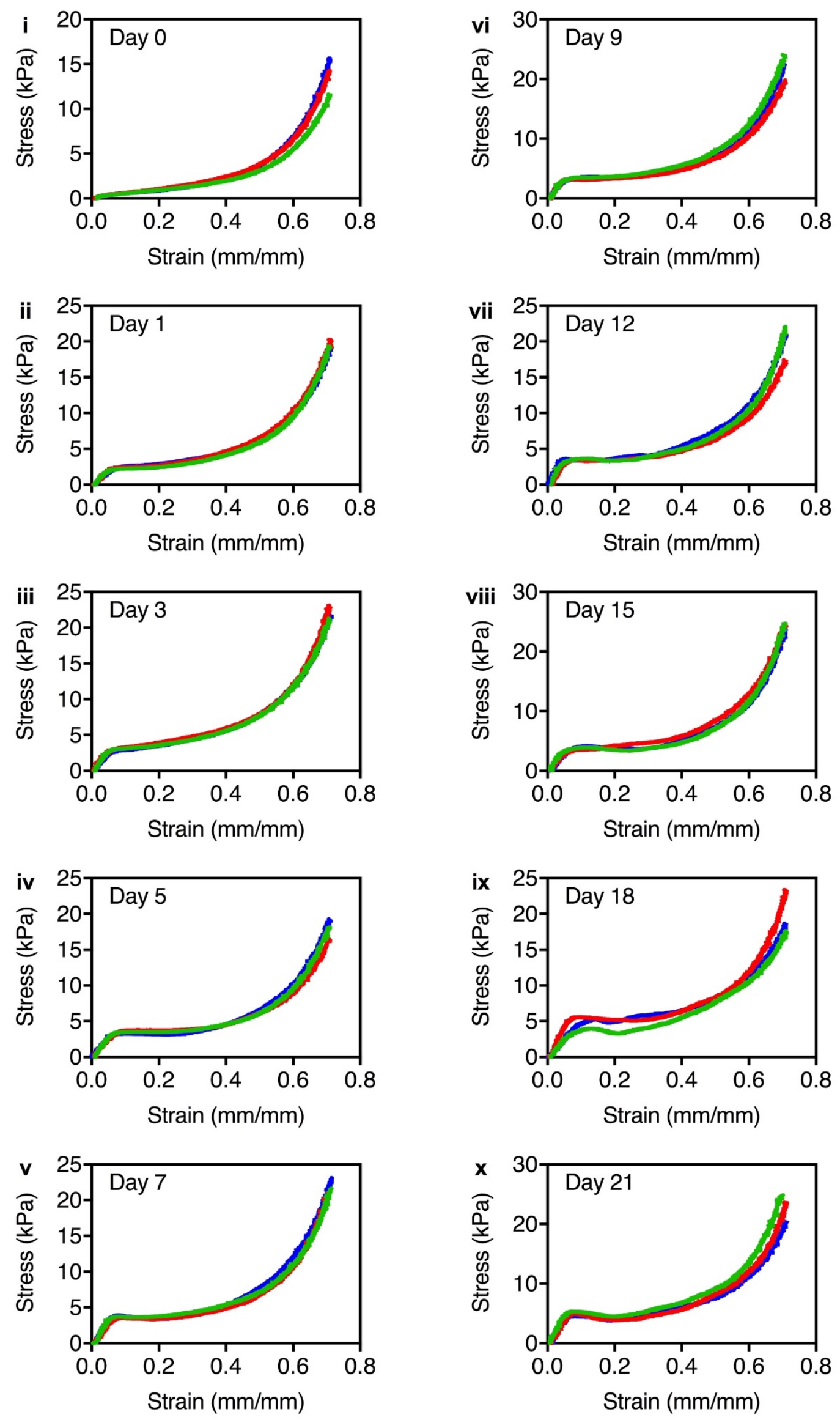

Figure S4. Stress-strain curves for 8CM10-75 produced on (i) day 0, (ii) day 1, (iii) day 3, (iv) day 5, (v) day 7, (vi) day 9, (vii) day 12, (viii) day 15, (ix) day 18, and (x) day 21. 

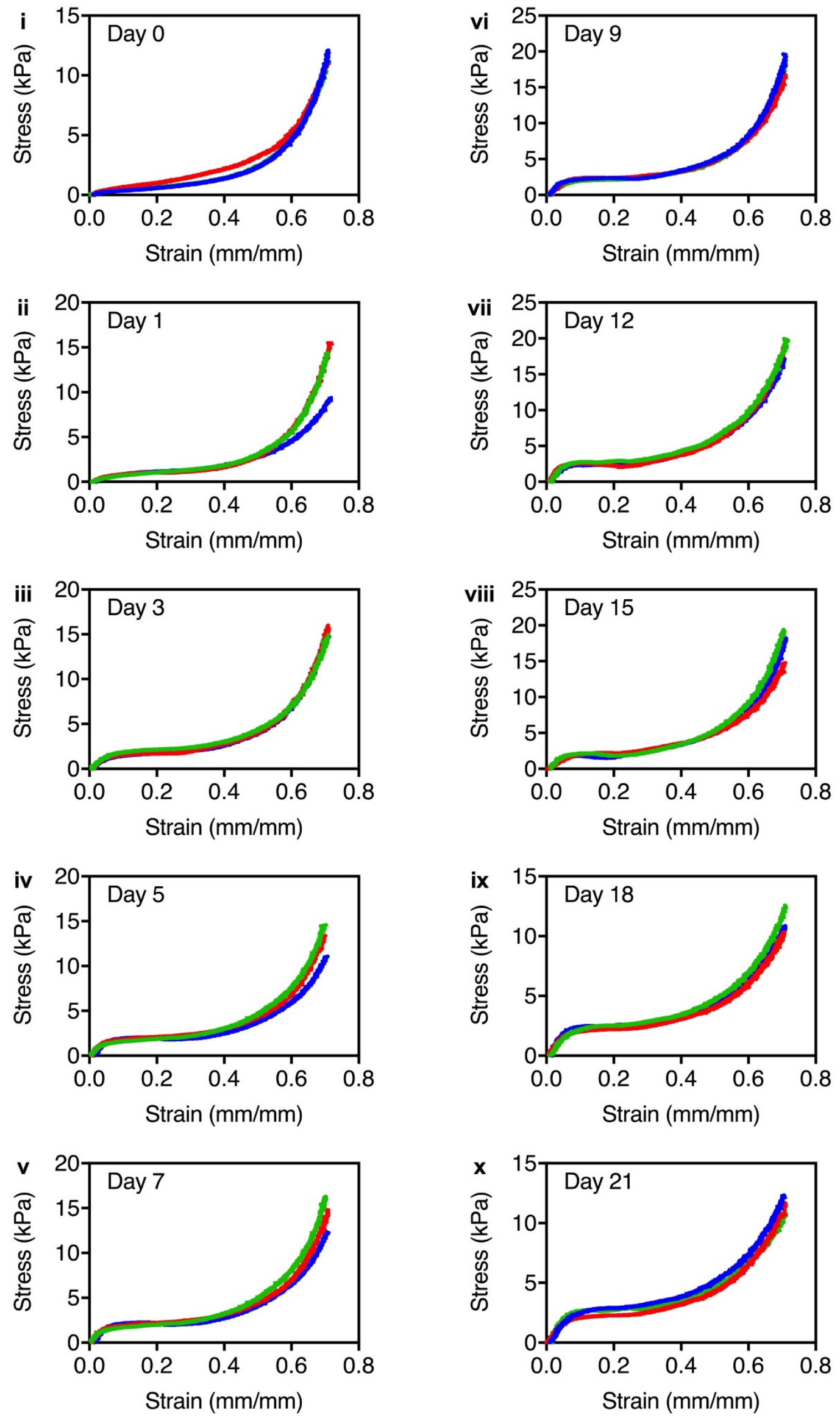

Figure S5. Stress-strain curves for 8CM20-75 produced on (i) day 0, (ii) day 1, (iii) day 3, (iv) day 5, (v) day 7, (vi) day 9, (vii) day 12, (viii) day 15, (ix) day 18, and (x) day 21. 

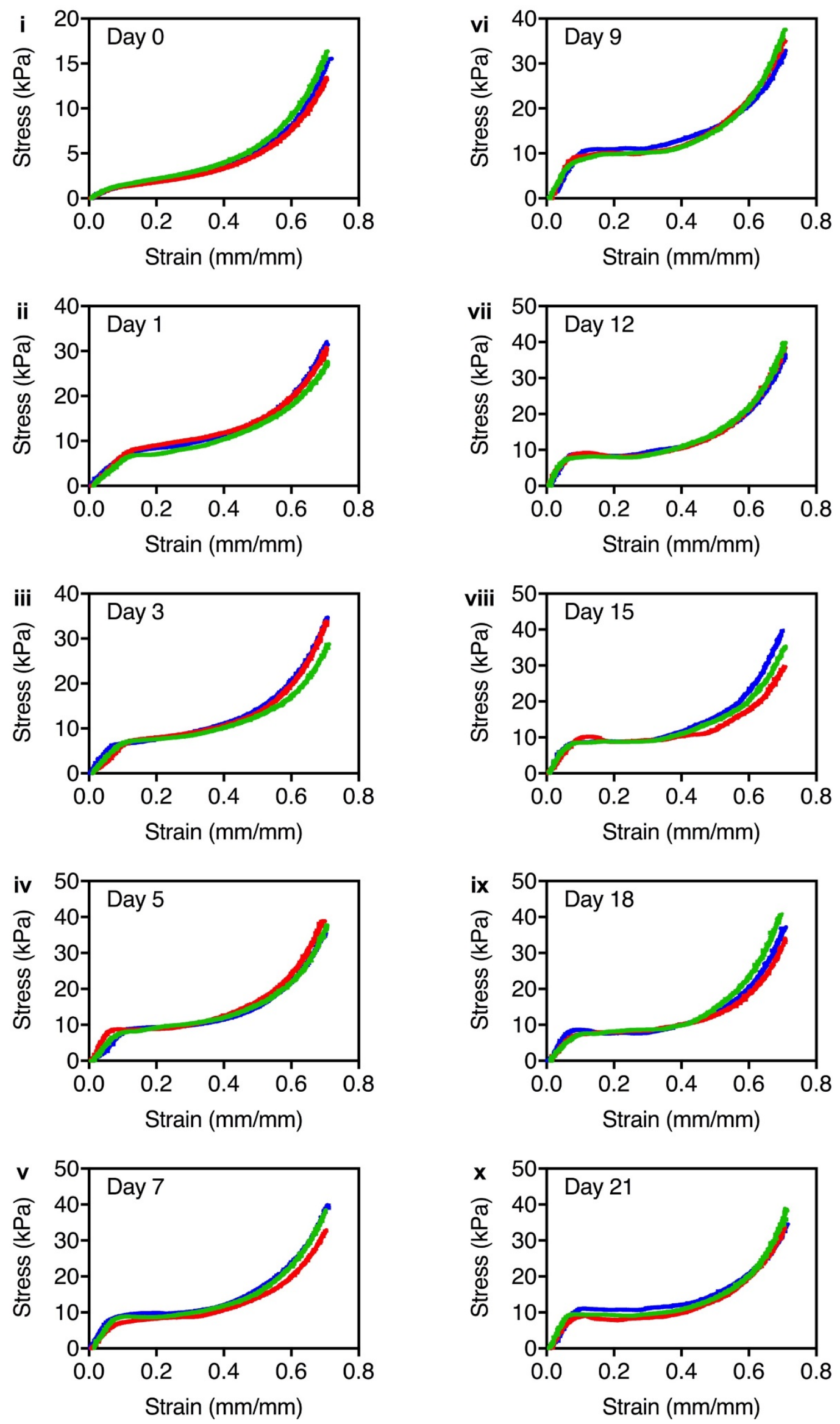

Figure S6. Stress-strain curves for 10CM0-75 produced on (i) day 0, (ii) day 1, (iii) day 3, (iv) day 5, (v) day 7, (vi) day 9, (vii) day 12, (viii) day 15, (ix) day 18 , and (x) day 21. 

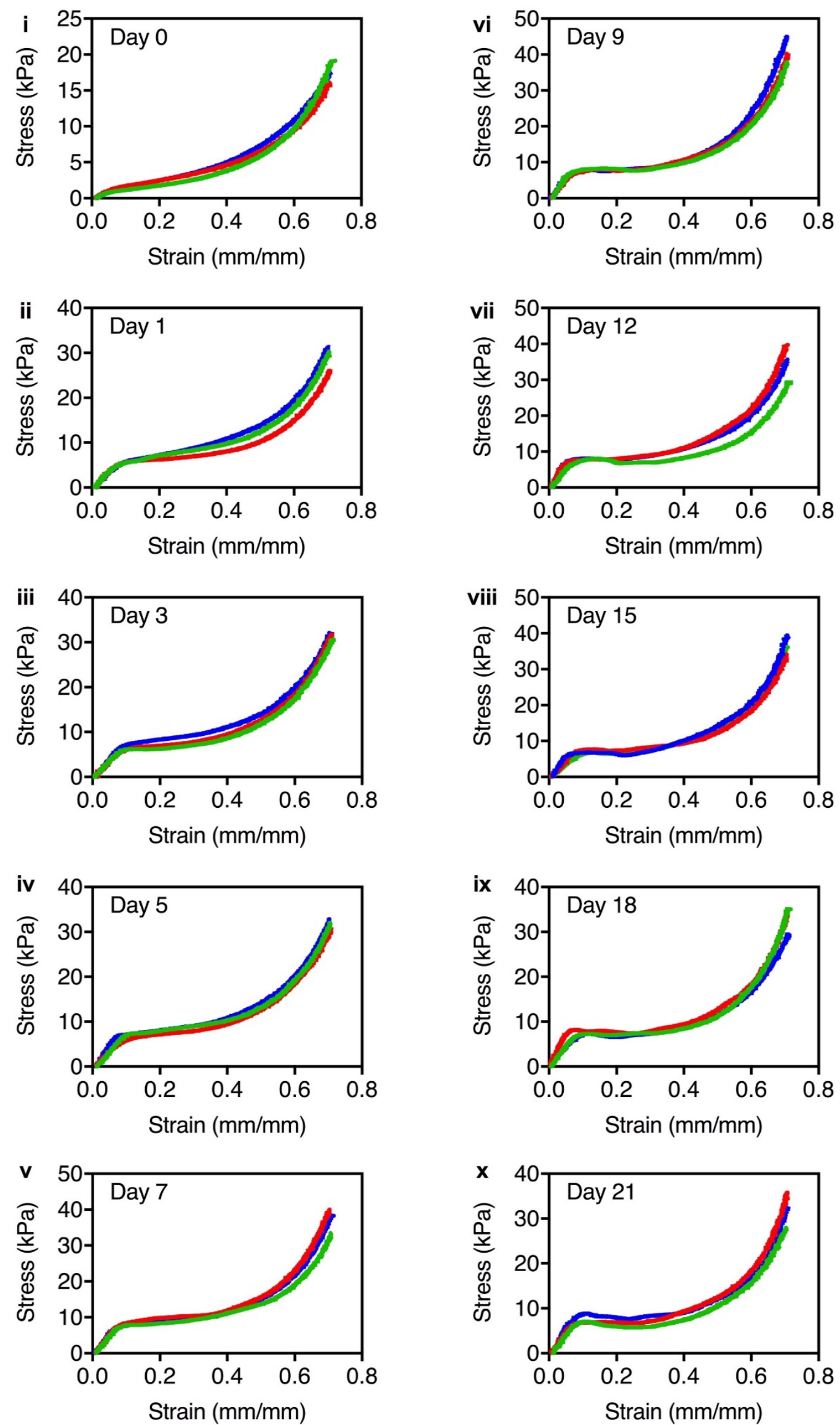

Figure S7. Stress-strain curves for 10CM5-75 produced on (i) day 0, (ii) day 1, (iii) day 3, (iv) day 5, (v) day 7, (vi) day 9, (vii) day 12, (viii) day 15, (ix) day 18 , and (x) day 21. 

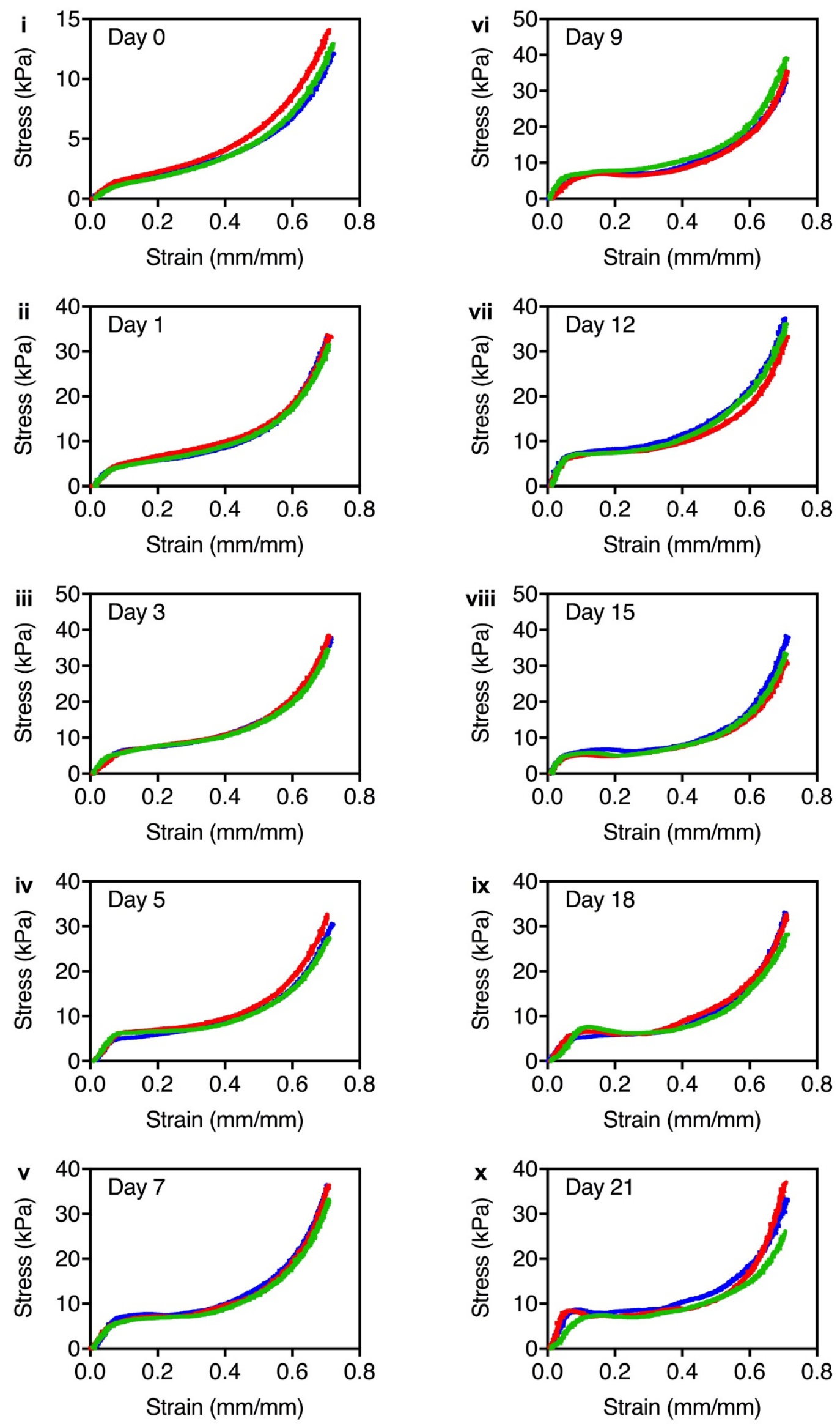

Figure S8. Stress-strain curves for 10CM10-75 produced on (i) day 0, (ii) day 1, (iii) day 3, (iv) day 5, (v) day 7, (vi) day 9, (vii) day 12, (viii) day 15, (ix) day 18 , and (x) day 21. 

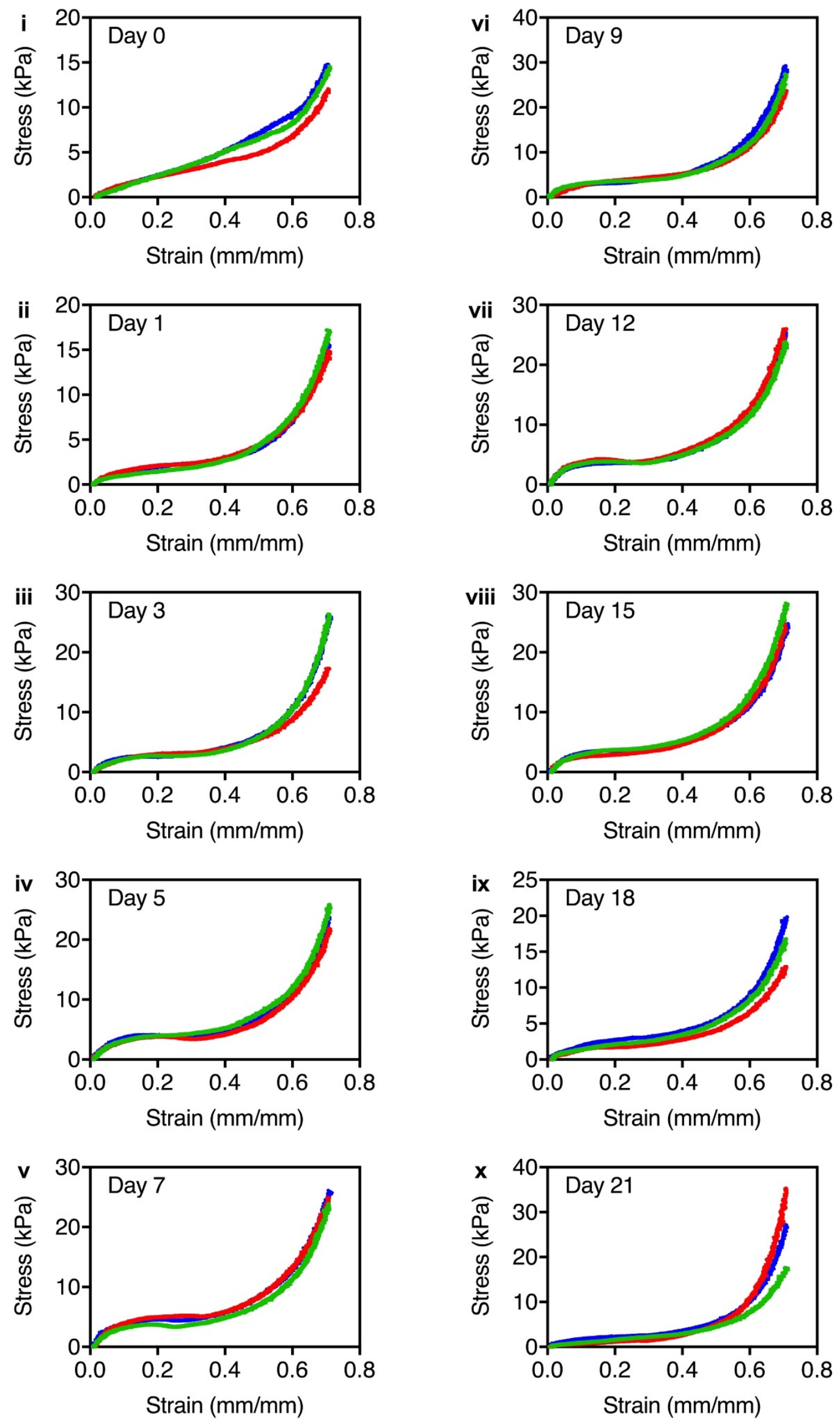

Figure S9. Stress-strain curves for 10CM20-75 produced on (i) day 0, (ii) day 1, (iii) day 3, (iv) day 5, (v) day 7, (vi) day 9, (vii) day 12, (viii) day 15, (ix) day 18 , and (x) day 21. 

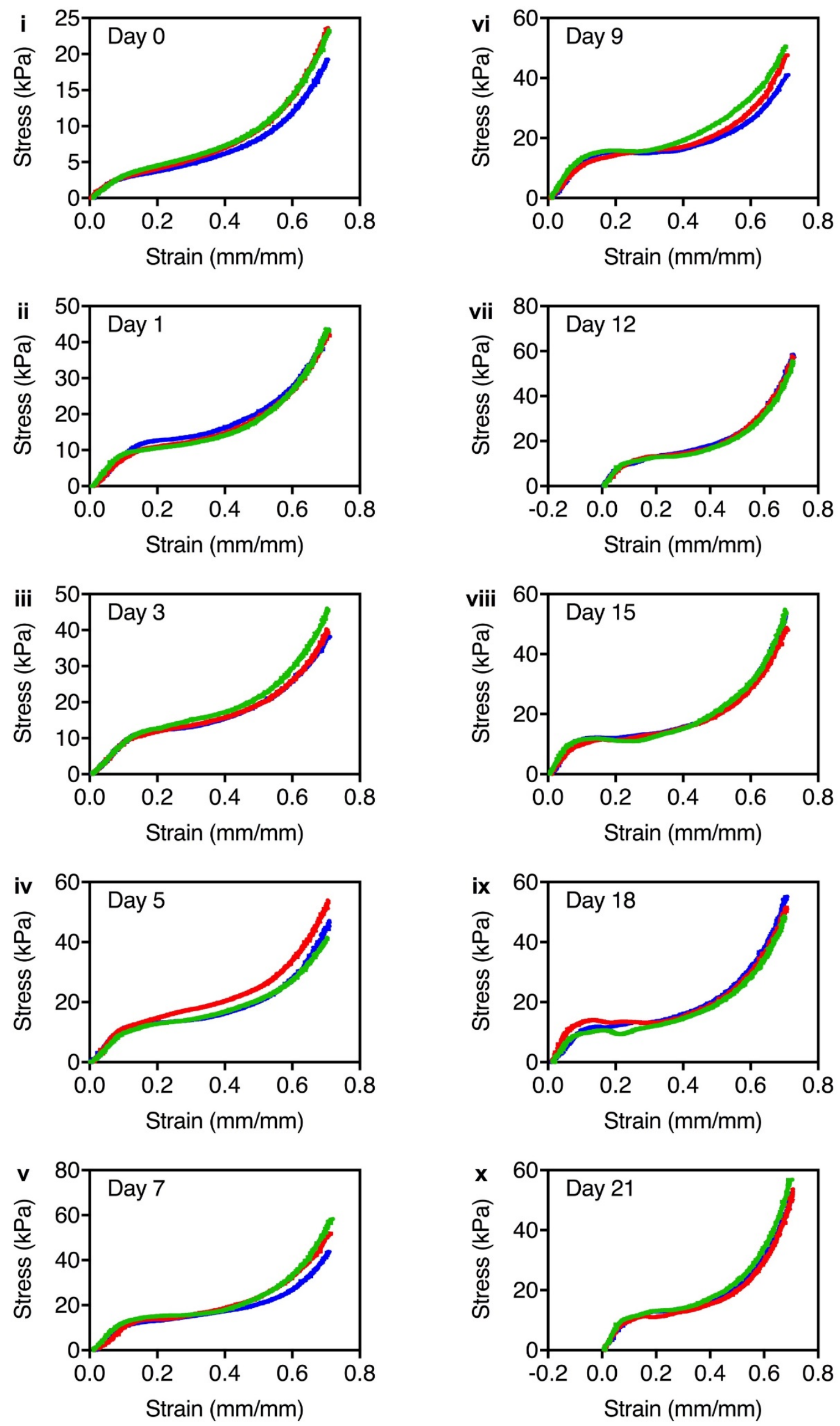

Figure S10. Stress-strain curves for 12CM0-75 produced on (i) day 0, (ii) day 1, (iii) day 3, (iv) day 5, (v) day 7, (vi) day 9, (vii) day 12, (viii) day 15, (ix) day 18, and (x) day 21. 

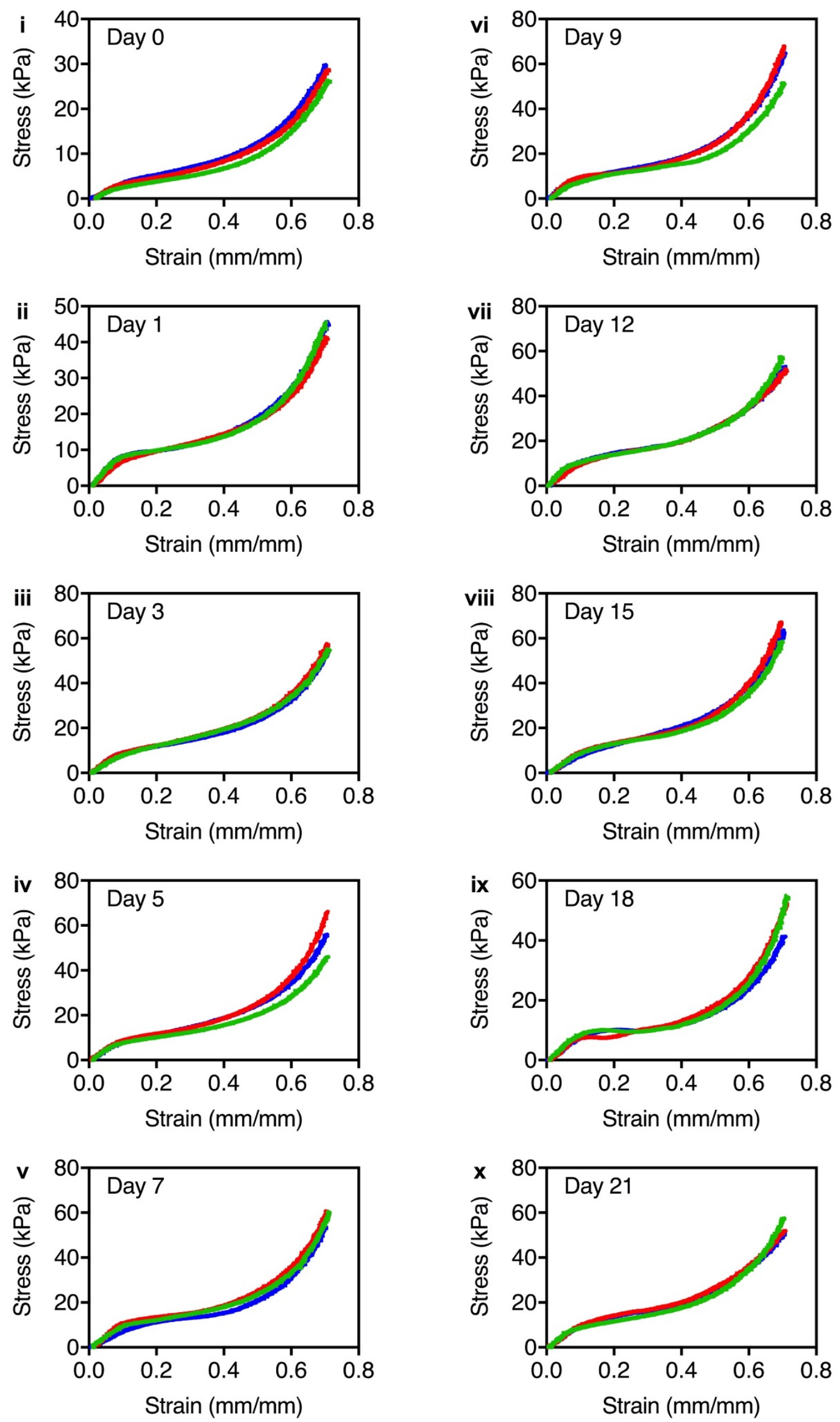

Figure S11. Stress-strain curves for 12CM5-75 produced on (i) day 0, (ii) day 1, (iii) day 3, (iv) day 5, (v) day 7, (vi) day 9, (vii) day 12, (viii) day 15, (ix) day 18 , and (x) day 21. 

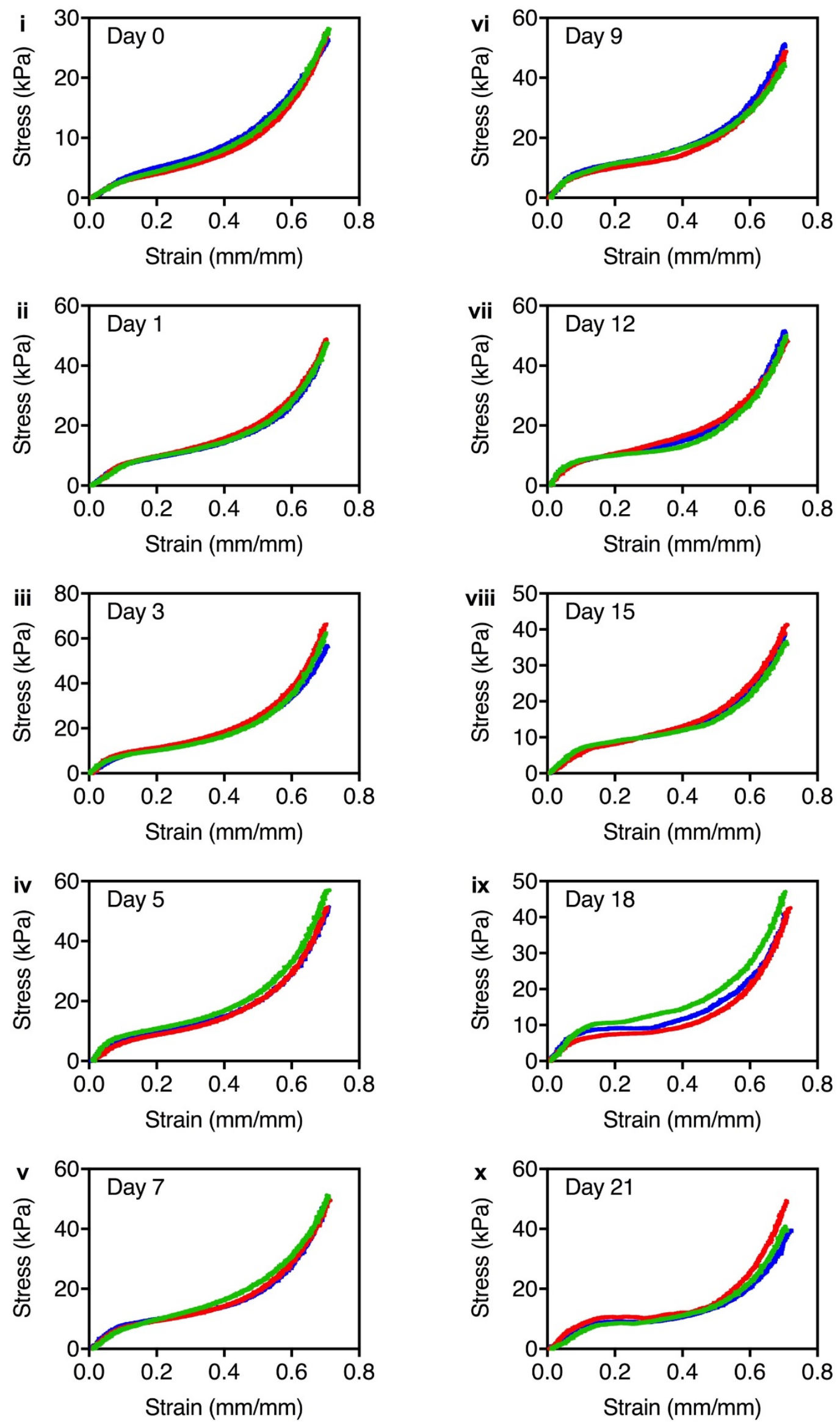

Figure S12. Stress-strain curves for 12CM10-75 produced on (i) day 0, (ii) day 1, (iii) day 3, (iv) day 5 , (v) day 7, (vi) day 9, (vii) day 12, (viii) day 15, (ix) day 18, and (x) day 21. 

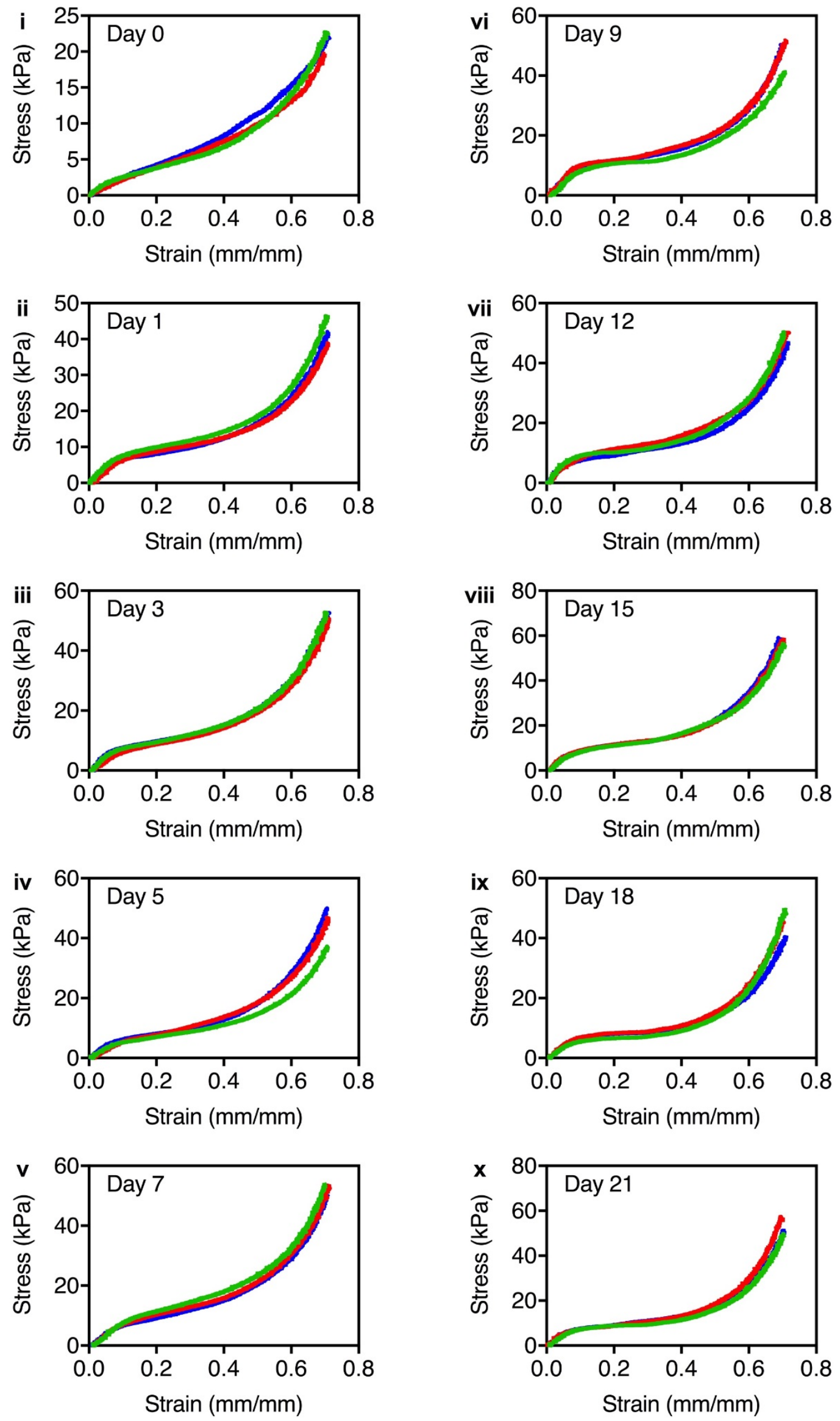

Figure S13. Stress-strain curves for 12CM20-75 produced on (i) day 0, (ii) day 1, (iii) day 3, (iv) day 5 , (v) day 7, (vi) day 9, (vii) day 12, (viii) day 15, (ix) day 18 , and (x) day 21. 

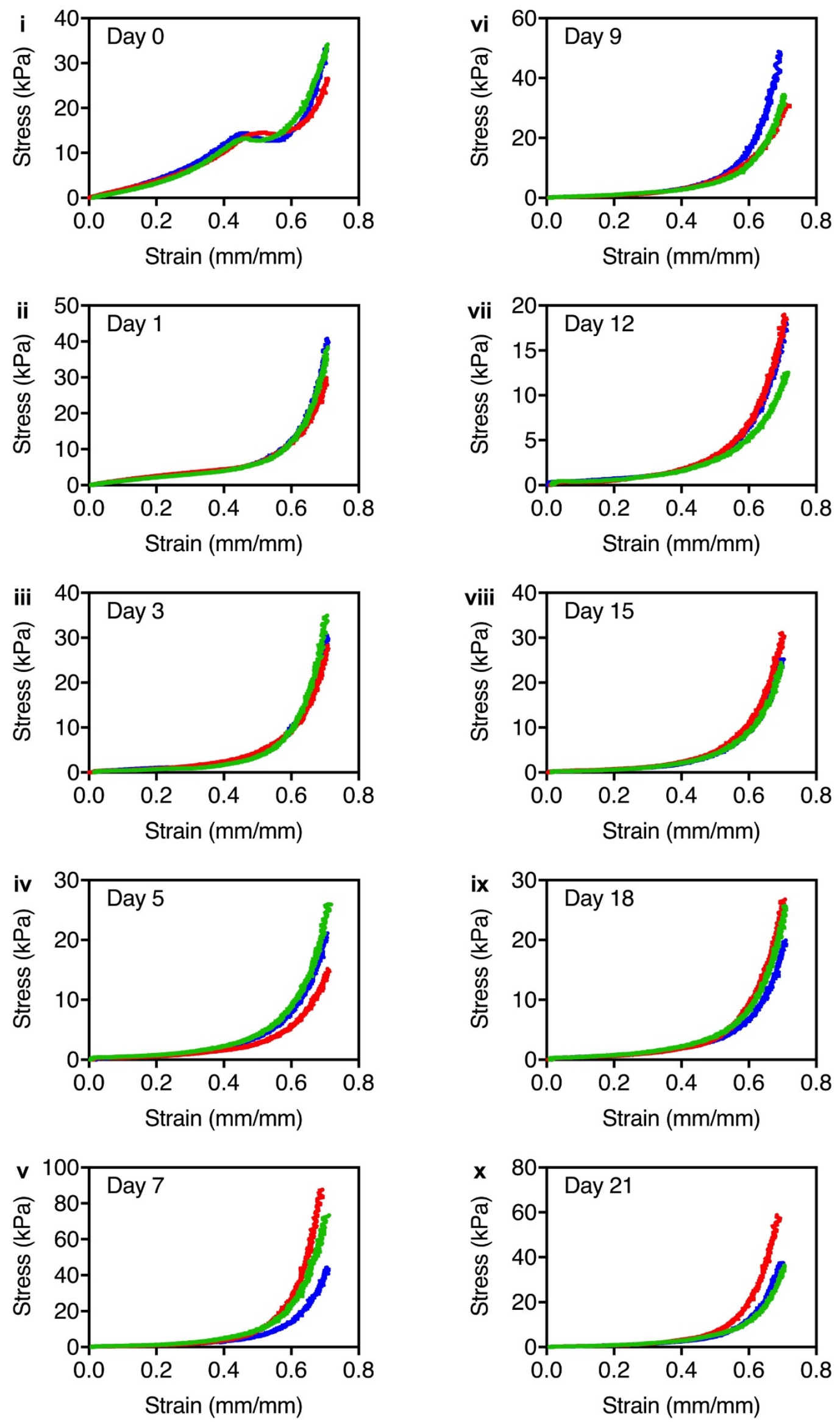

Figure S14. Stress-strain curves for 8CM0-50 produced on (i) day 0, (ii) day 1, (iii) day 3, (iv) day 5, (v) day 7, (vi) day 9, (vii) day 12, (viii) day 15, (ix) day 18, and (x) day 21. 

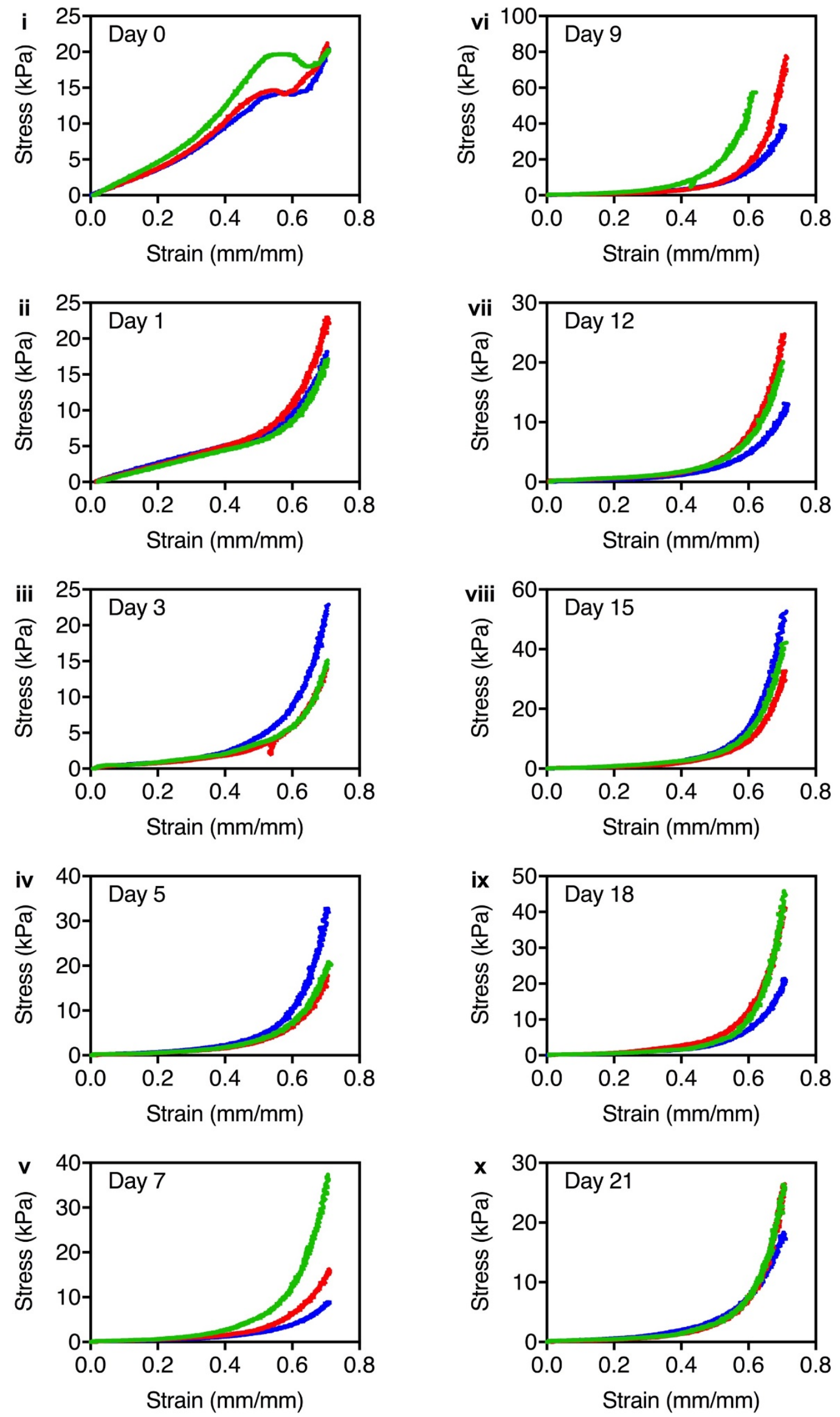

Figure S15. Stress-strain curves for 8CM5-50 produced on (i) day 0, (ii) day 1, (iii) day 3, (iv) day 5, (v) day 7, (vi) day 9, (vii) day 12, (viii) day 15, (ix) day 18 , and (x) day 21. 

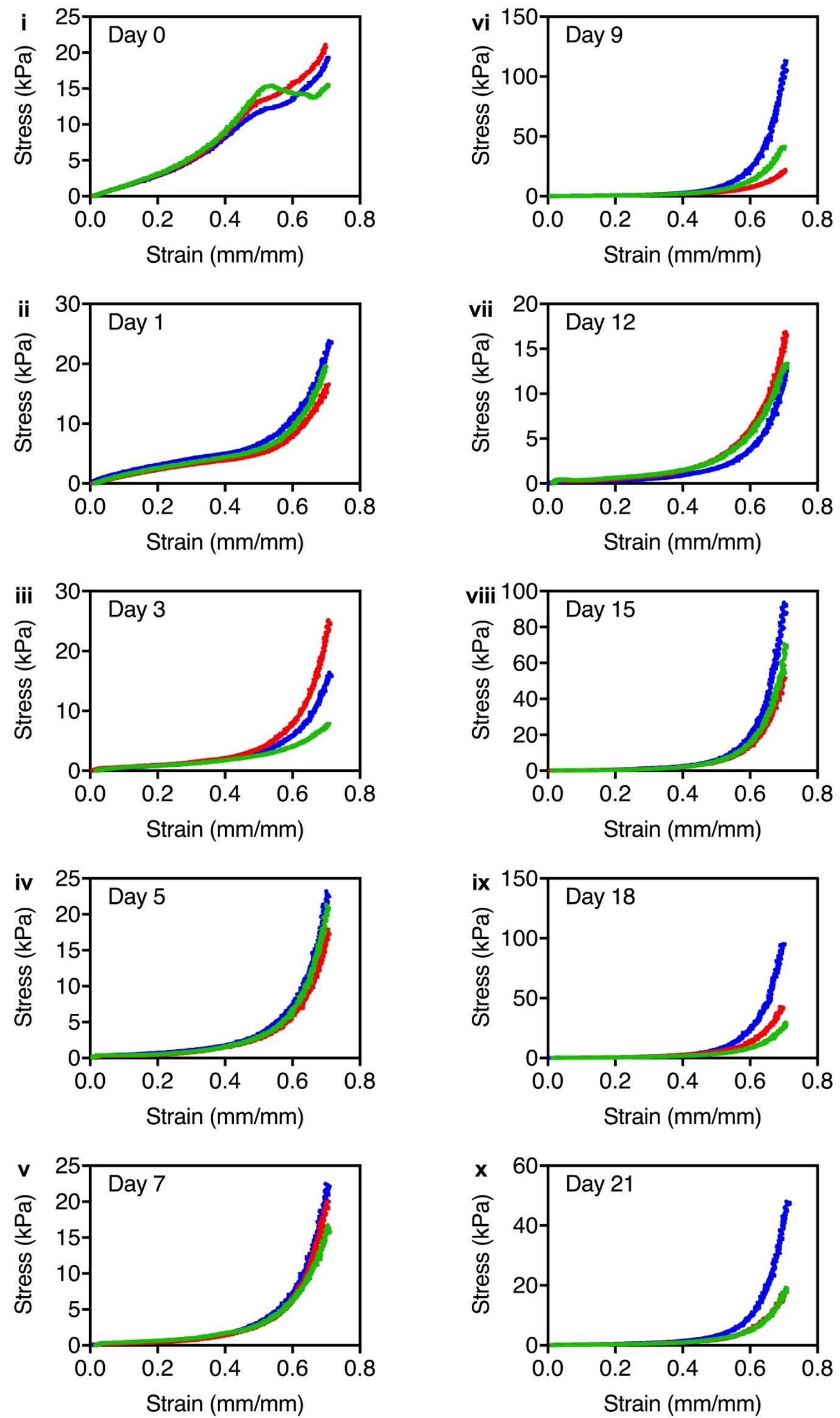

Figure S16. Stress-strain curves for 8CM10-50 produced on (i) day 0, (ii) day 1, (iii) day 3, (iv) day 5, (v) day 7, (vi) day 9, (vii) day 12, (viii) day 15, (ix) day 18, and (x) day 21. 

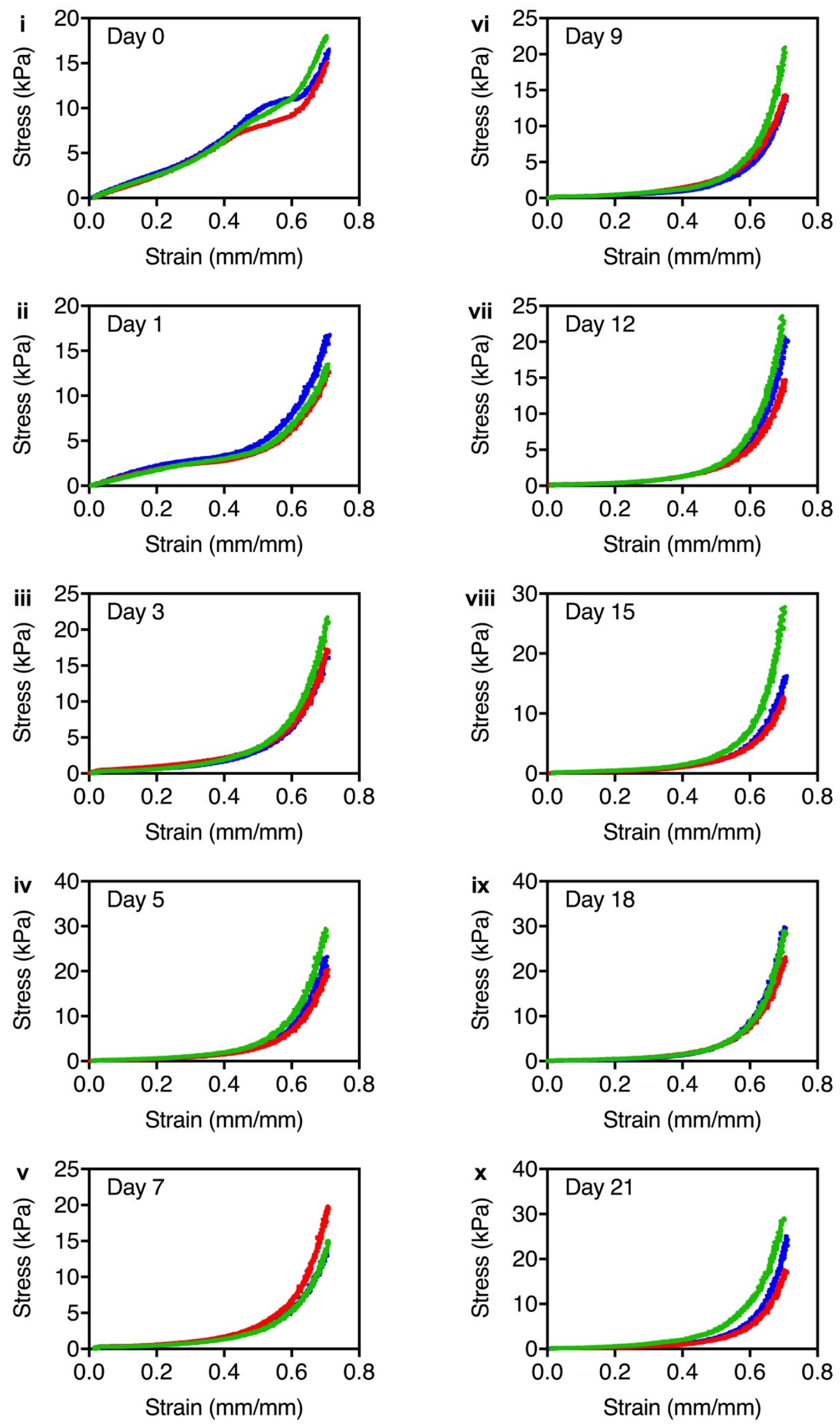

Figure S17. Stress-strain curves for 8CM20-50 produced on (i) day 0, (ii) day 1, (iii) day 3, (iv) day 5, (v) day 7, (vi) day 9, (vii) day 12, (viii) day 15, (ix) day 18, and (x) day 21. 

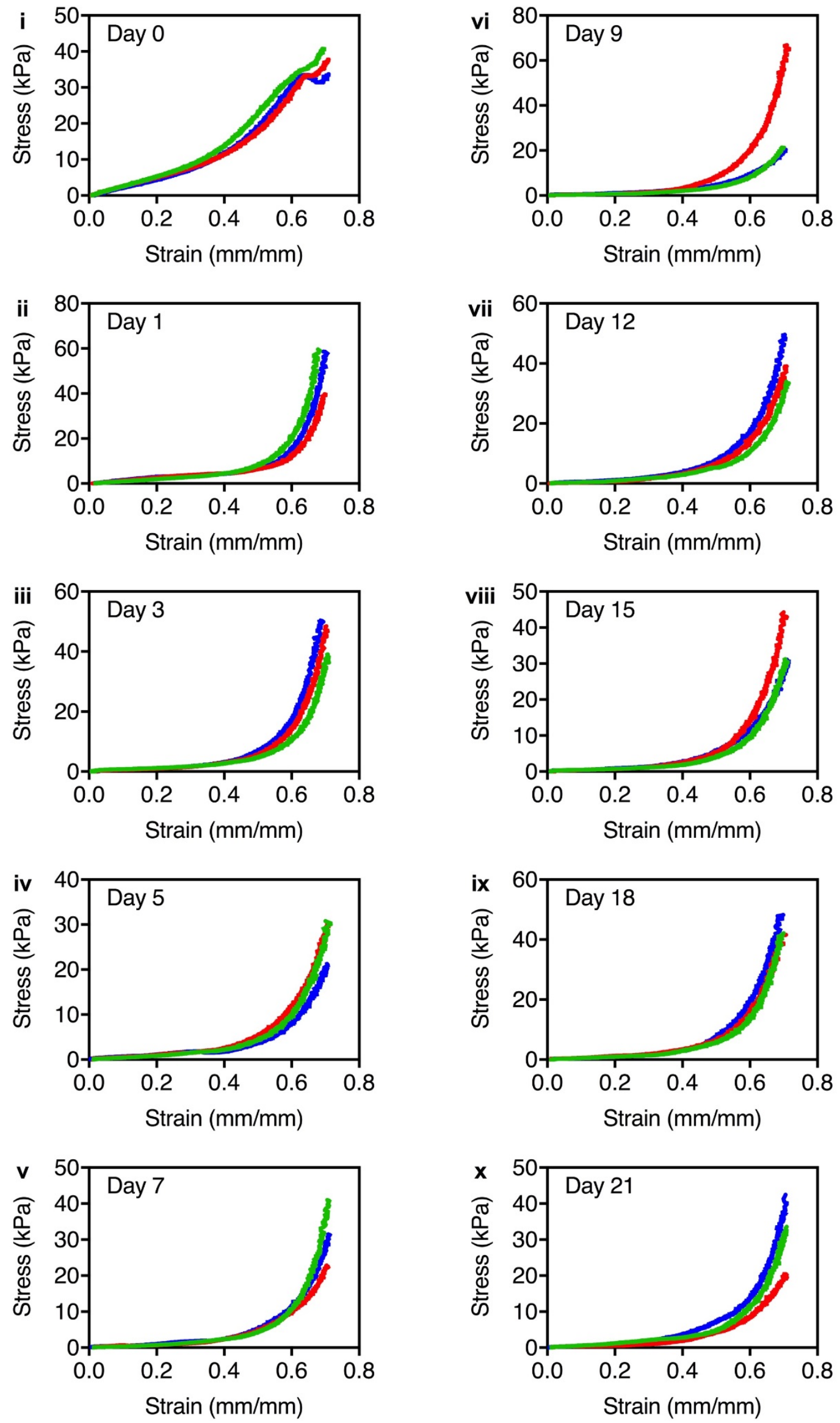

Figure S18. Stress-strain curves for 10CM0-50 produced on (i) day 0, (ii) day 1, (iii) day 3, (iv) day 5, (v) day 7, (vi) day 9, (vii) day 12, (viii) day 15, (ix) day 18, and (x) day 21. 

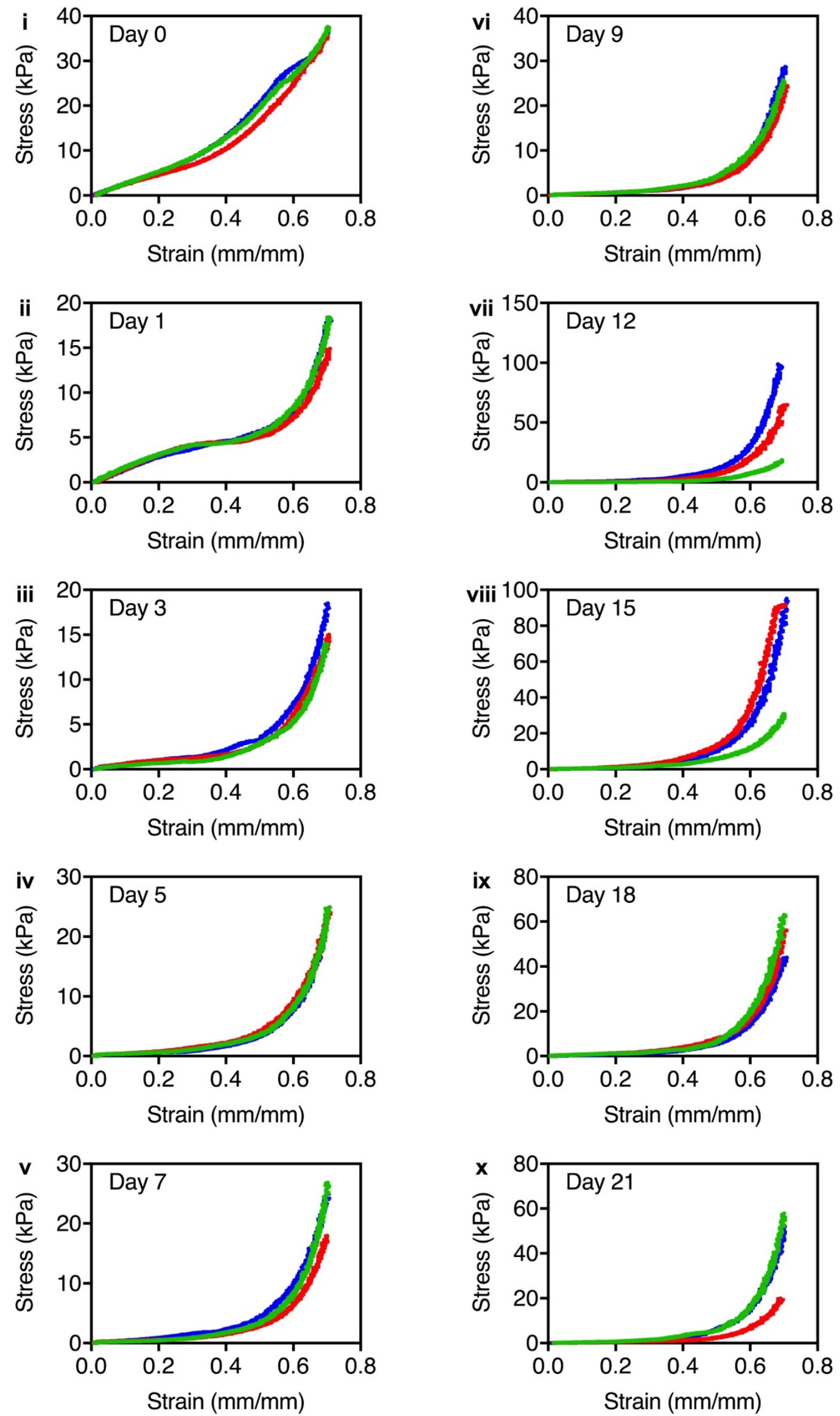

Figure S19. Stress-strain curves for 10CM5-50 produced on (i) day 0, (ii) day 1, (iii) day 3, (iv) day 5, (v) day 7, (vi) day 9, (vii) day 12, (viii) day 15, (ix) day 18, and (x) day 21. 

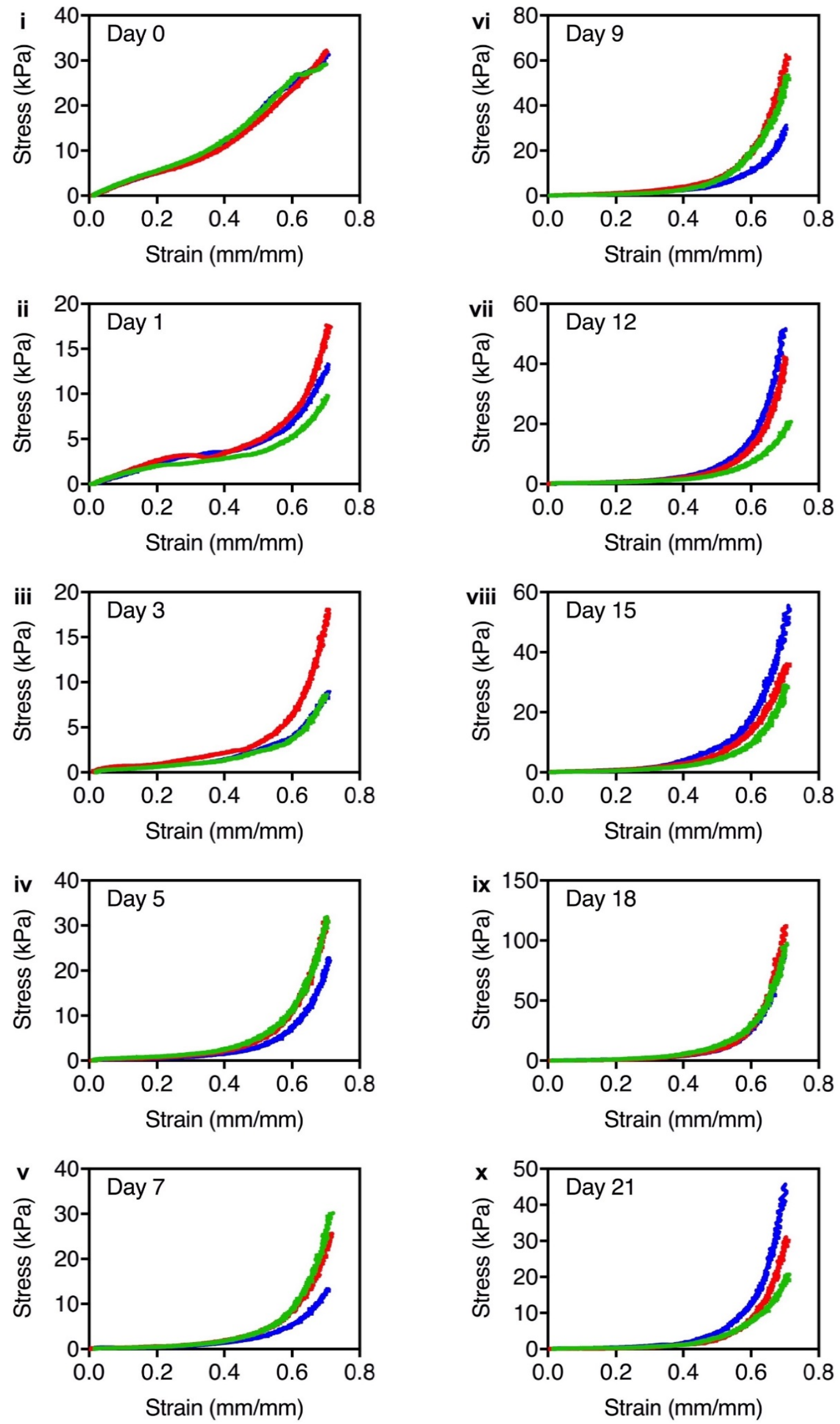

Figure S20. Stress-strain curves for 10CM10-50 produced on (i) day 0, (ii) day 1, (iii) day 3, (iv) day 5, (v) day 7, (vi) day 9, (vii) day 12, (viii) day 15, (ix) day 18, and (x) day 21. 

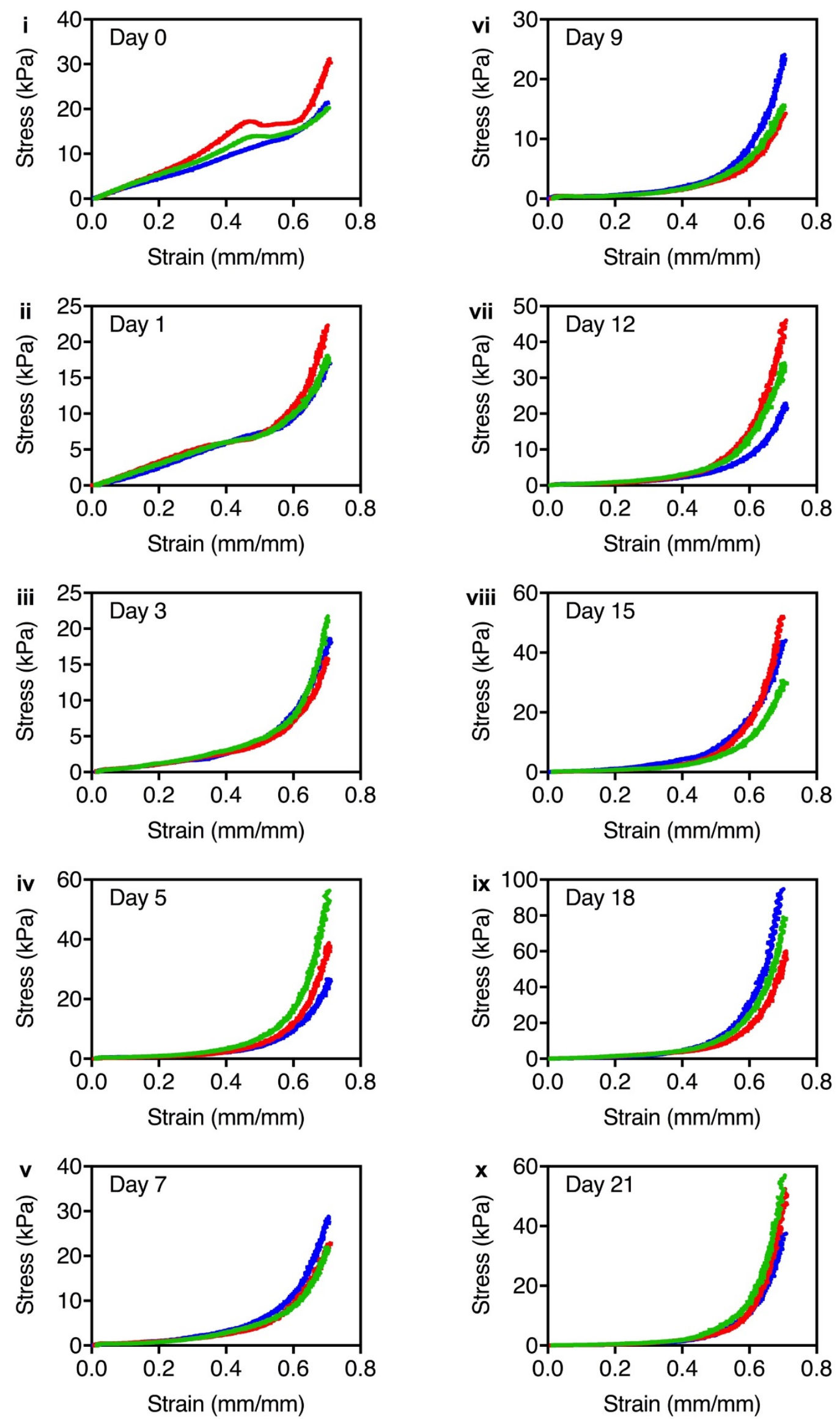

Figure S21. Stress-strain curves for 10CM20-50 produced on (i) day 0, (ii) day 1, (iii) day 3, (iv) day 5, (v) day 7, (vi) day 9, (vii) day 12, (viii) day 15, (ix) day 18, and (x) day 21. 

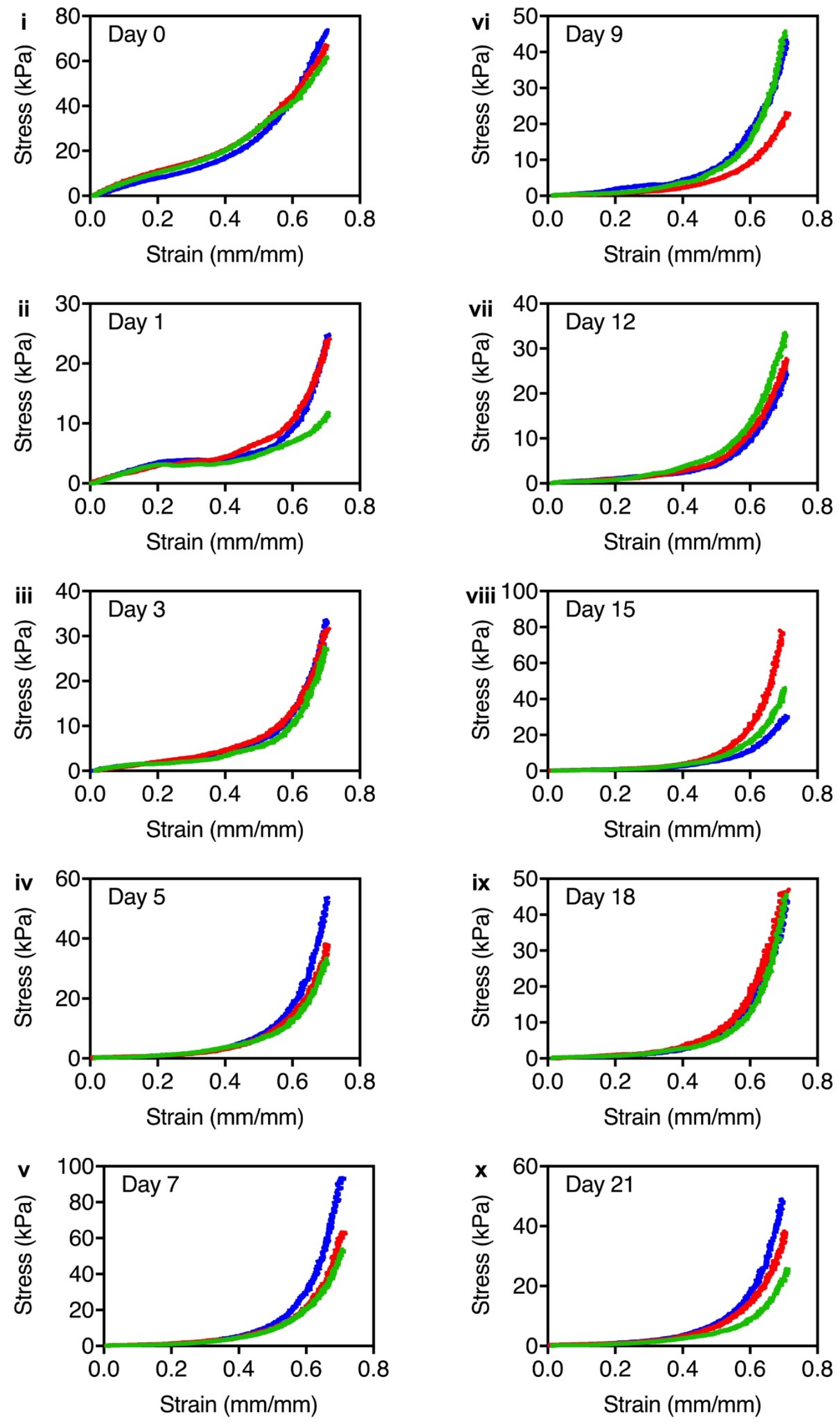

Figure S22. Stress-strain curves for 12CM0-50 produced on (i) day 0, (ii) day 1, (iii) day 3, (iv) day 5, (v) day 7, (vi) day 9, (vii) day 12, (viii) day 15, (ix) day 18, and (x) day 21. 

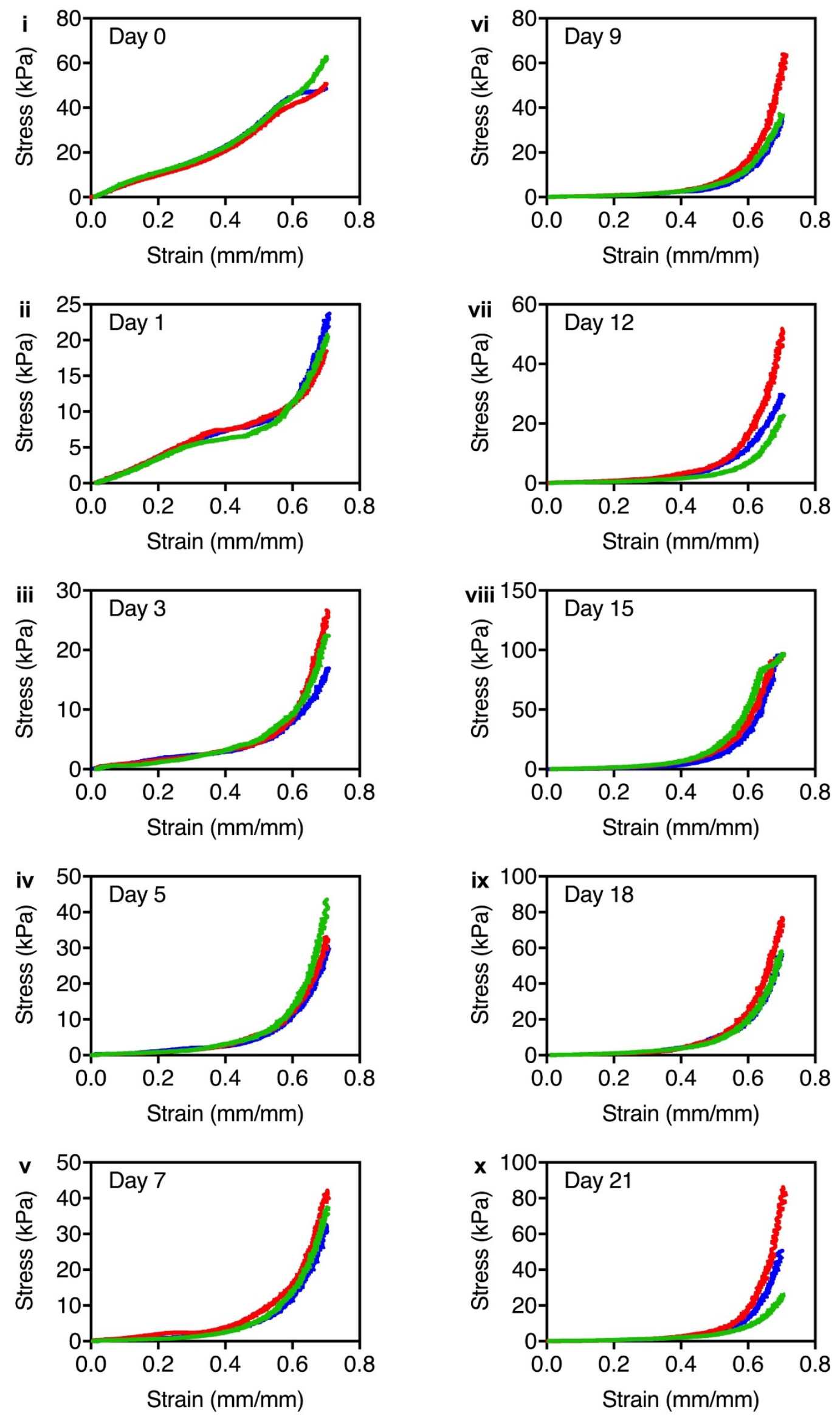

Figure S23. Stress-strain curves for 12CM5-50 produced on (i) day 0, (ii) day 1, (iii) day 3, (iv) day 5, (v) day 7, (vi) day 9, (vii) day 12, (viii) day 15, (ix) day 18, and (x) day 21. 

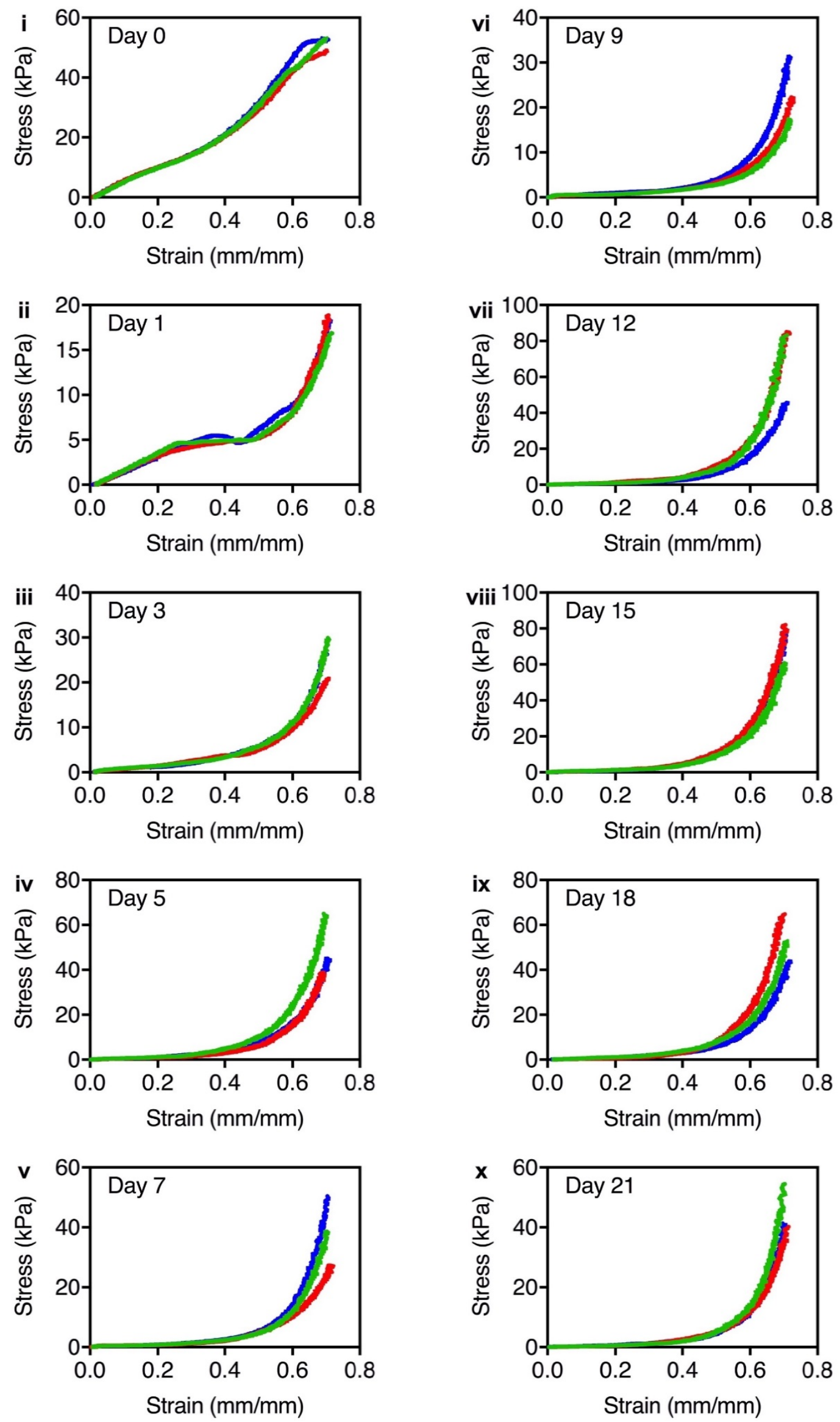

Figure S24. Stress-strain curves for 12CM10-50 produced on (i) day 0, (ii) day 1, (iii) day 3, (iv) day 5, (v) day 7, (vi) day 9, (vii) day 12, (viii) day 15, (ix) day 18, and (x) day 21. 

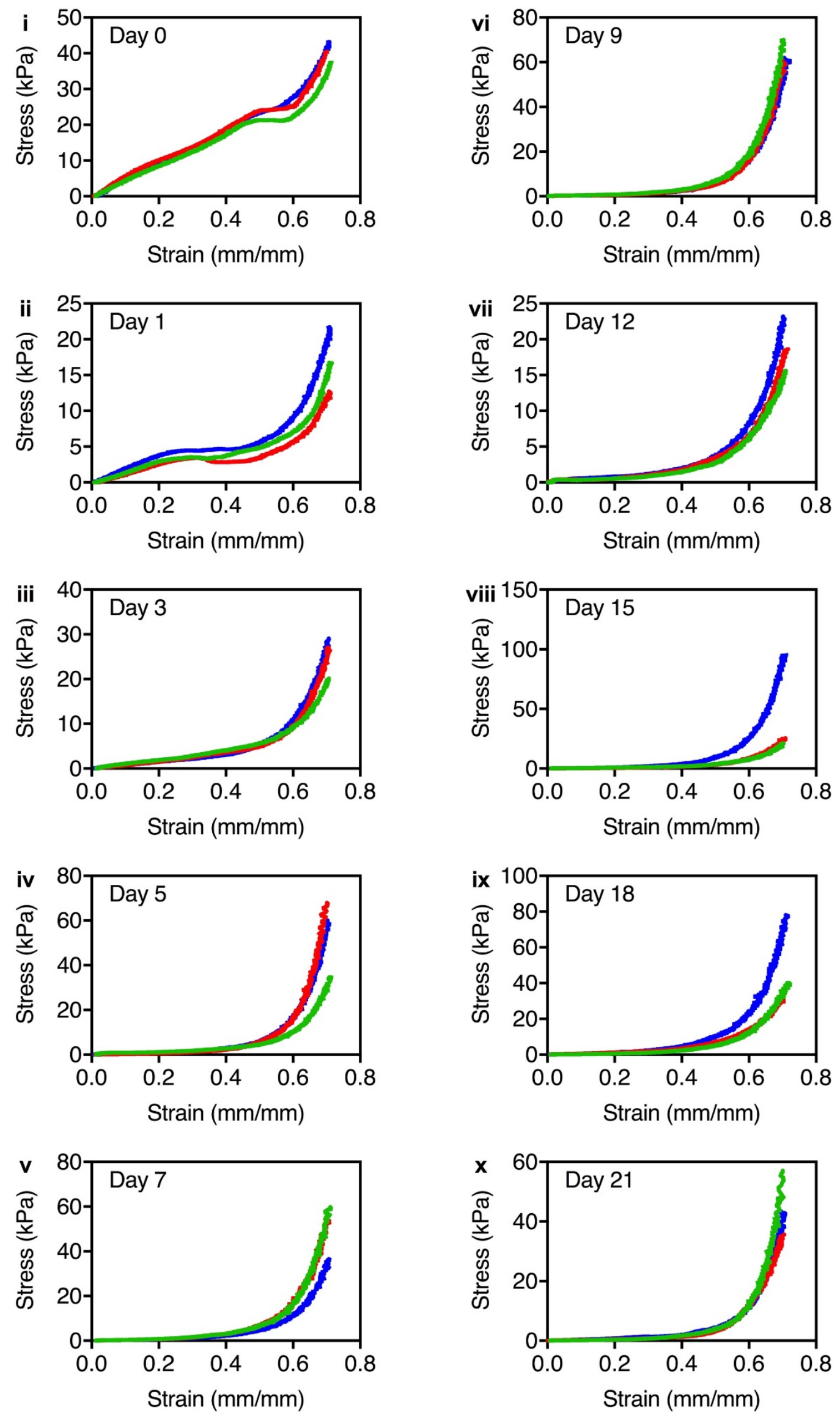

Figure S25. Stress-strain curves for 12CM20-50 produced on (i) day 0, (ii) day 1, (iii) day 3, (iv) day 5, (v) day 7, (vi) day 9, (vii) day 12, (viii) day 15, (ix) day 18, and (x) day 21. 


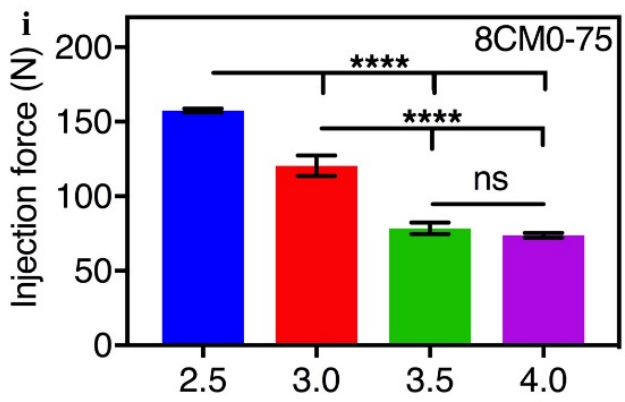

Catheter internal diameter $(\mathrm{mm})$

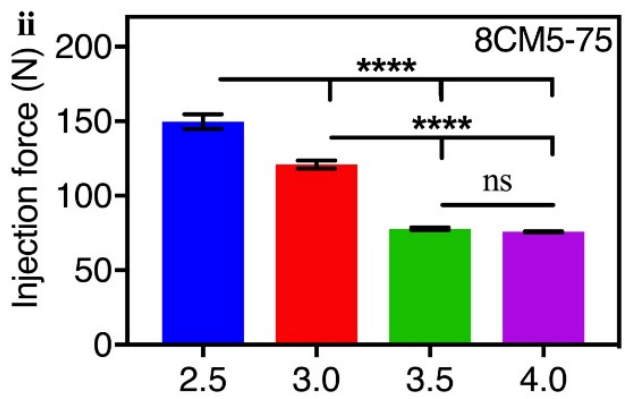

Catheter internal diameter $(\mathrm{mm})$

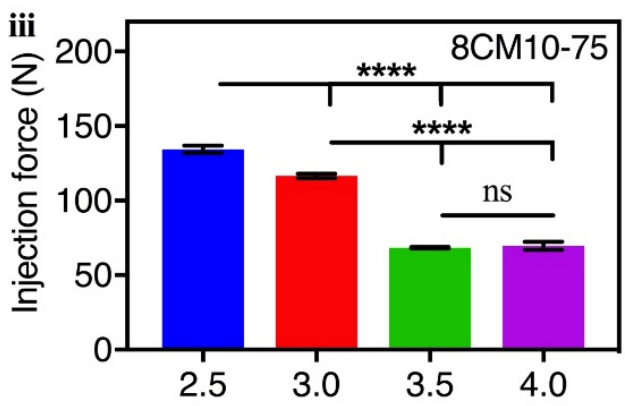

Catheter internal diameter $(\mathrm{mm})$

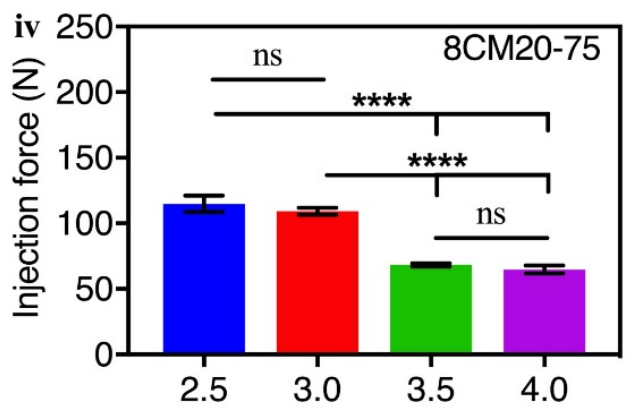

Catheter internal diameter $(\mathrm{mm})$

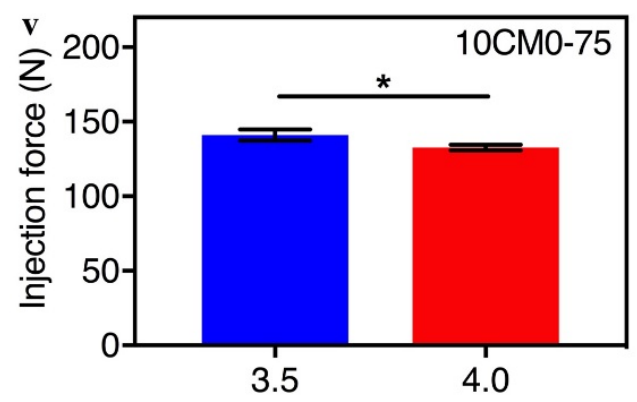

Catheter internal diameter $(\mathrm{mm})$

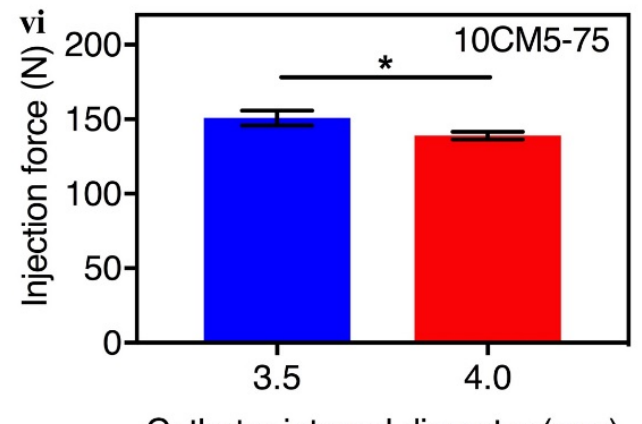

Catheter internal diameter $(\mathrm{mm})$

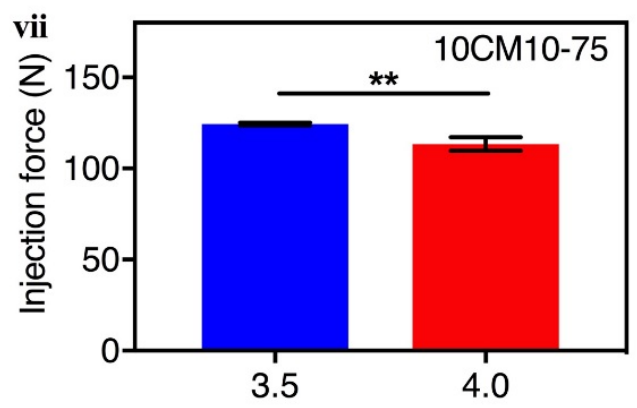

Catheter internal diameter $(\mathrm{mm})$

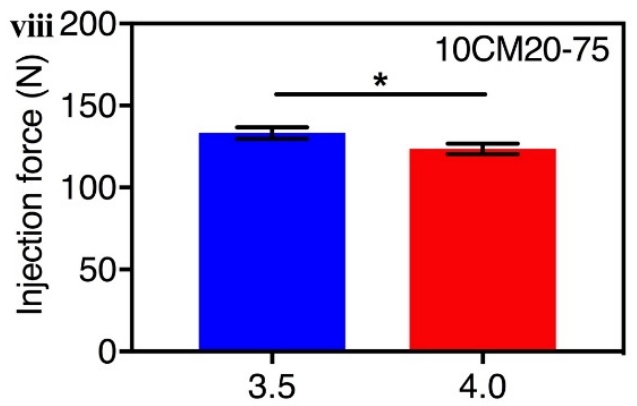

Catheter internal diameter $(\mathrm{mm})$

Figure S26. The injection force required to inject (i) 8CM0-75, (ii) 8CM5-75, (iii) 8CM10-75, (iv) 8CM20-75, (v) 10CM0-75, (vi) 10CM5-75, (vii) 10CM10-75, and (viii) 10CM20-75 through catheters of varying internal diameter. $P$-values were determined by one-way analysis of variance with HolmSidak's multiple comparisons tests for (i)-(iv) and unpaired Student's t-tests for (v)-(viii) $\left({ }^{*} P<0.05\right.$, $* * P<0.01, * * * P<0.001, * * * * P<0.0001)$. Data are means $\pm \mathrm{SD}(\mathrm{n}=3)$. 

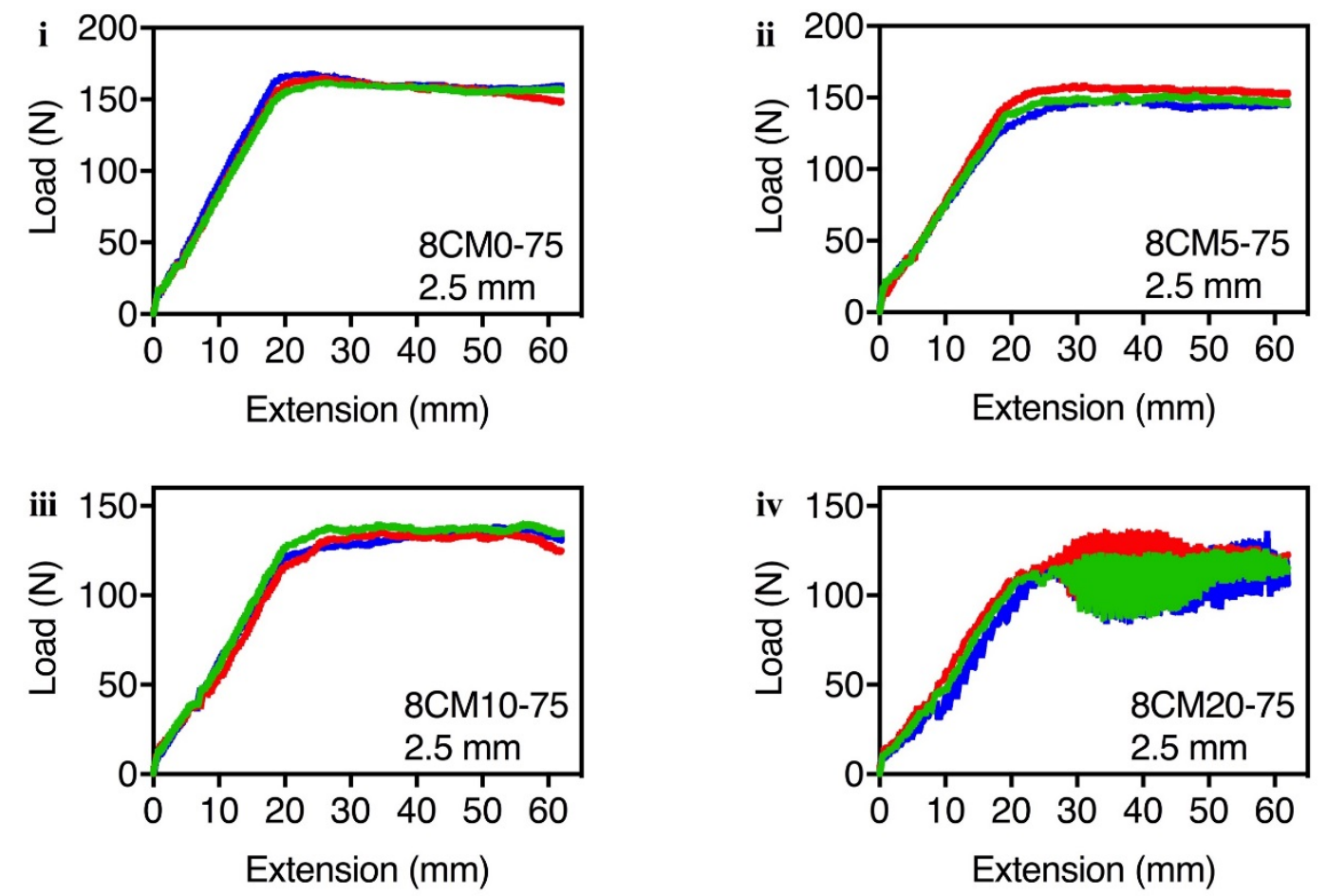

Figure S27. Force profiles of (i) 8CM0-75, (ii) 8CM5-75, (iii) 8CM10-75, (iv) 8CM20-75 through a 2.5 mm ID catheter.
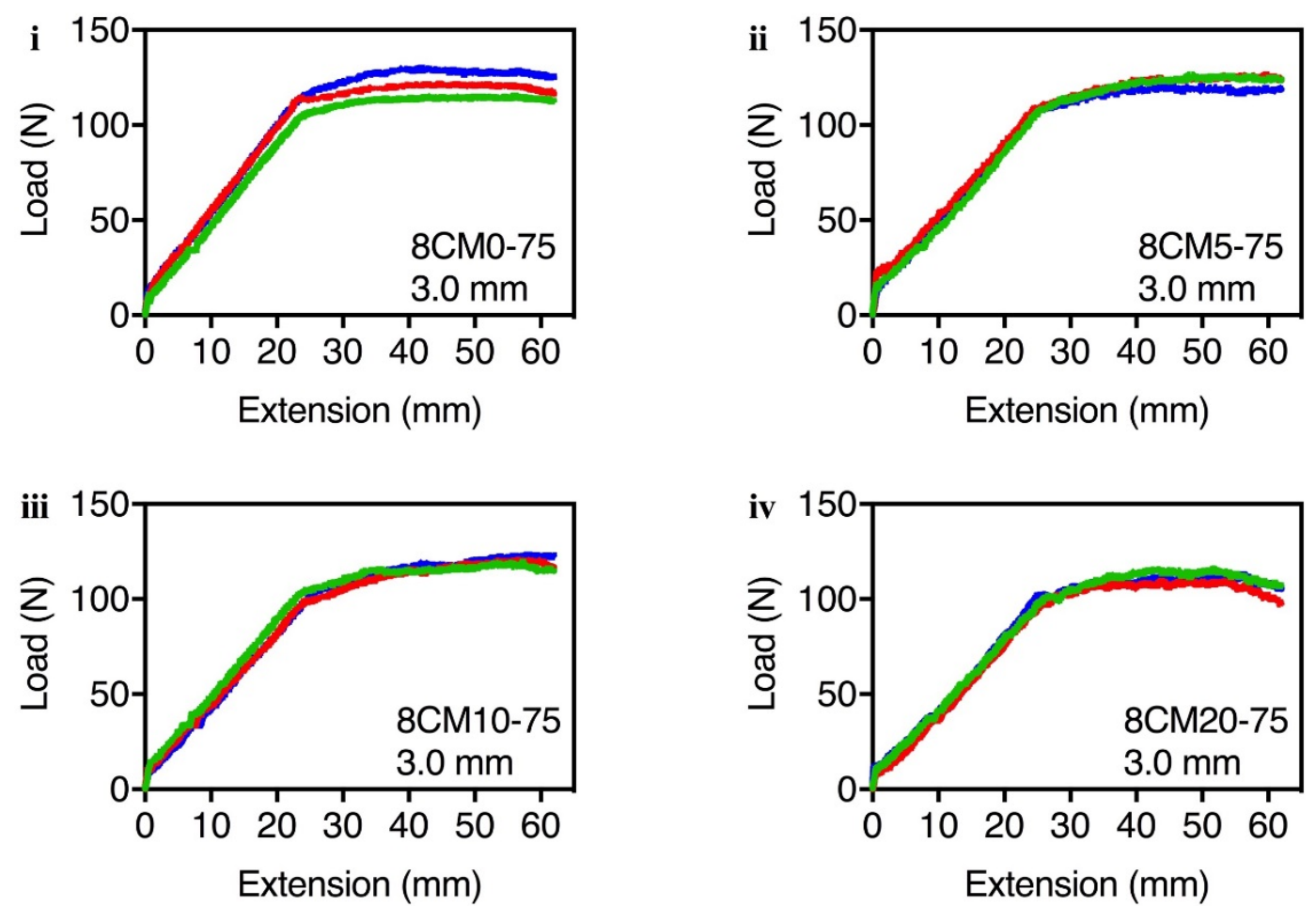

Figure S28. Force profiles of (i) 8CM0-75, (ii) 8CM5-75, (iii) 8CM10-75, (iv) 8CM20-75 through a 3.0 mm ID catheter. 

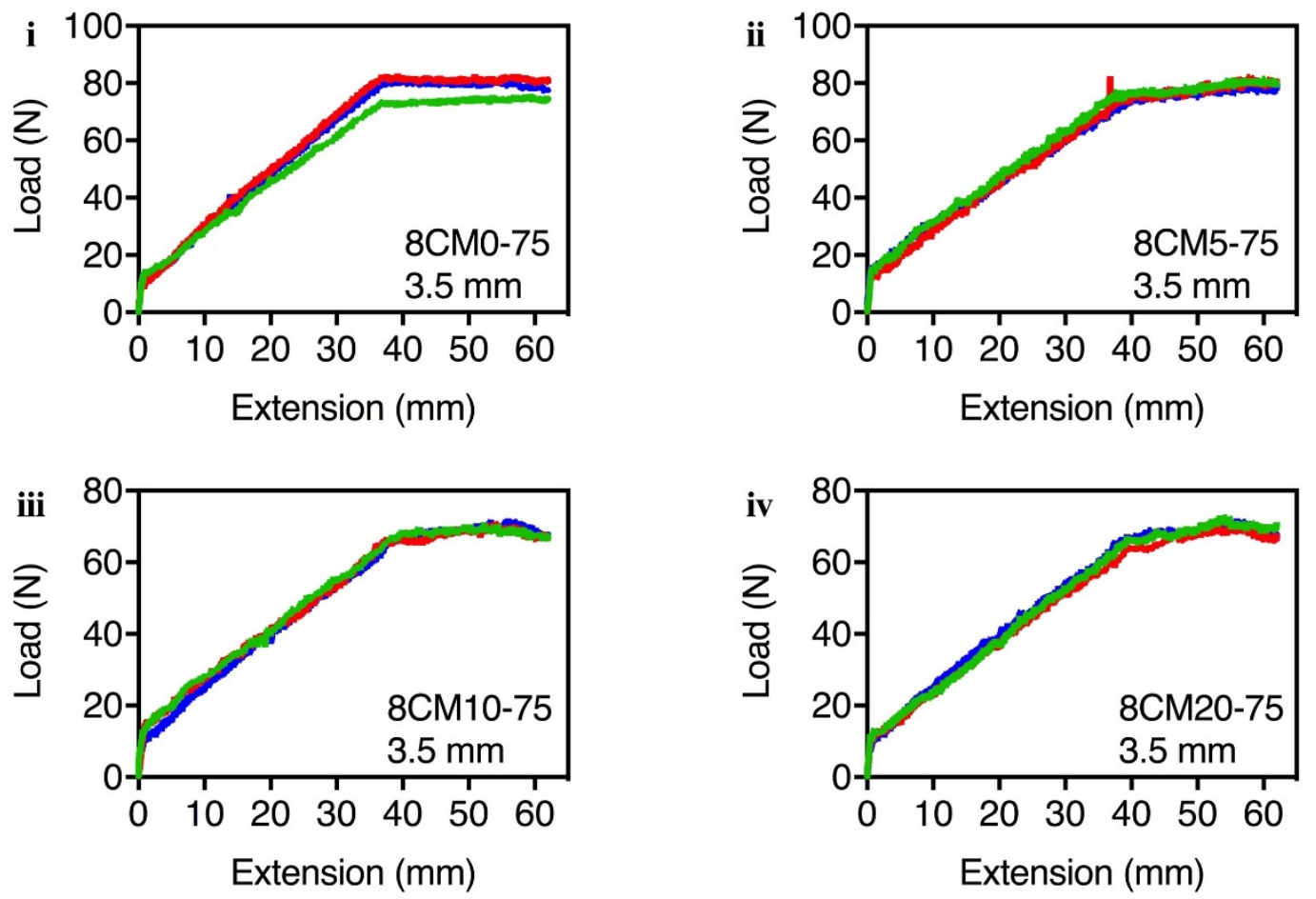

Figure S29. Force profiles of (i) 8CM0-75, (ii) 8CM5-75, (iii) 8CM10-75, (iv) 8CM20-75 through a 3.5 mm ID catheter.
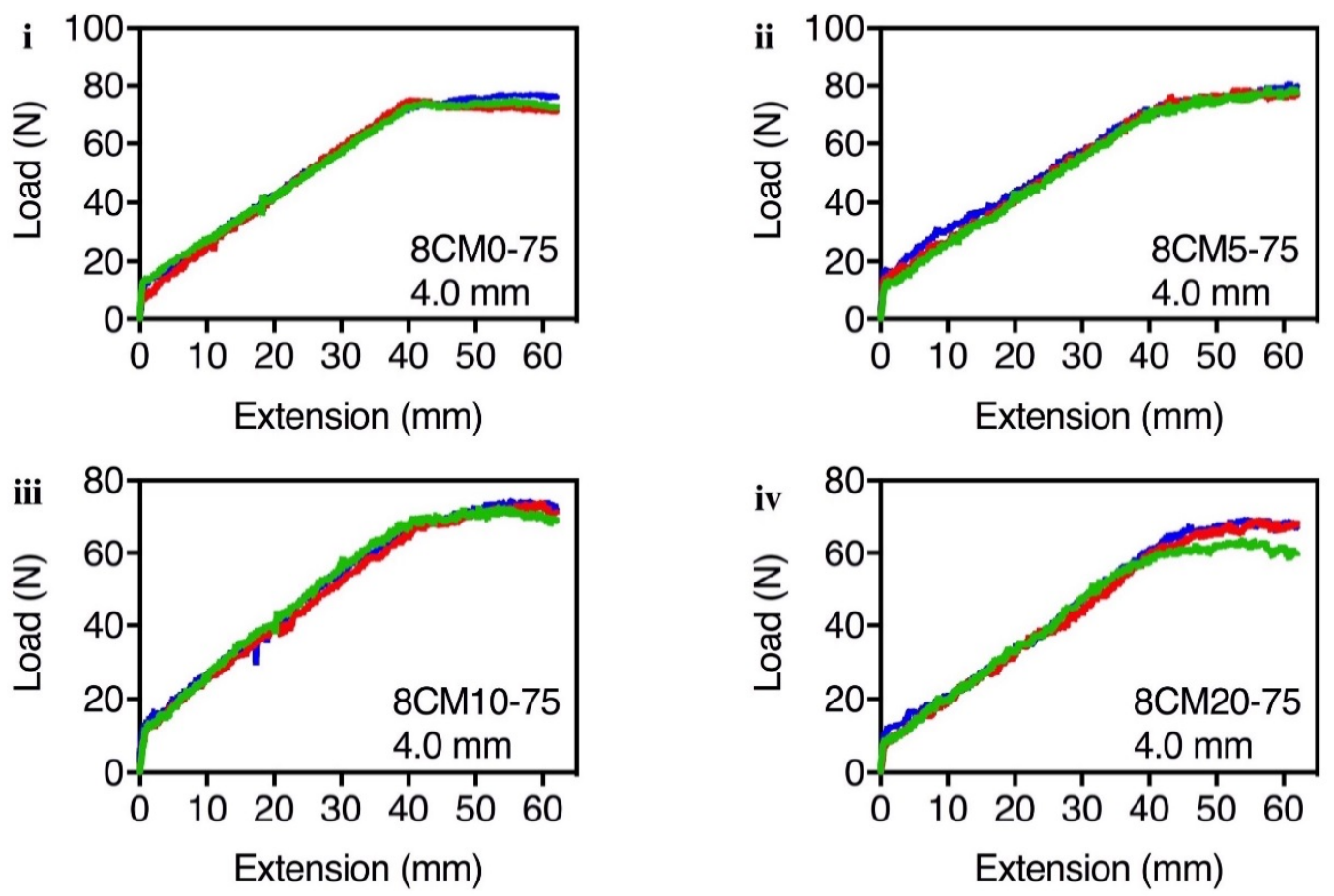

Figure S30. Force profiles of (i) 8CM0-75, (ii) 8CM5-75, (iii) 8CM10-75, (iv) 8CM20-75 through a 4.0 $\mathrm{mm}$ ID catheter. 


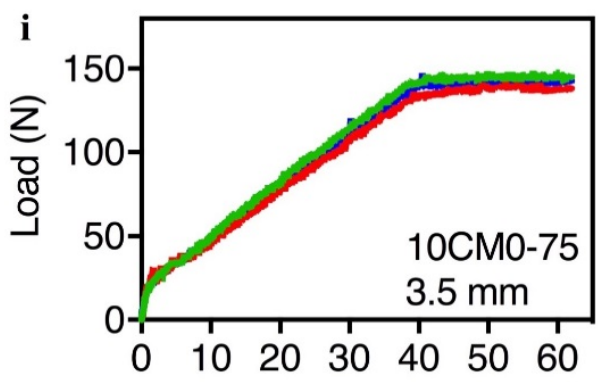

Extension (mm)

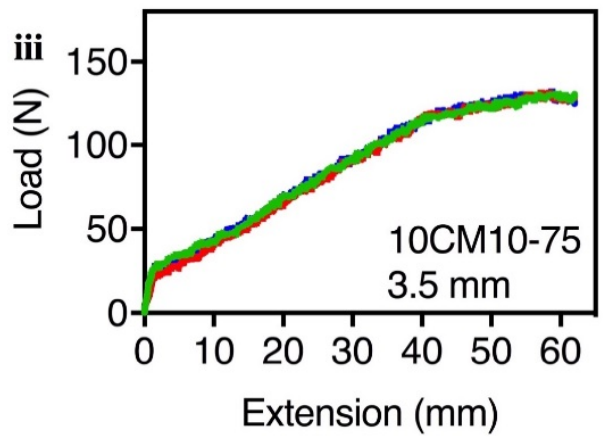

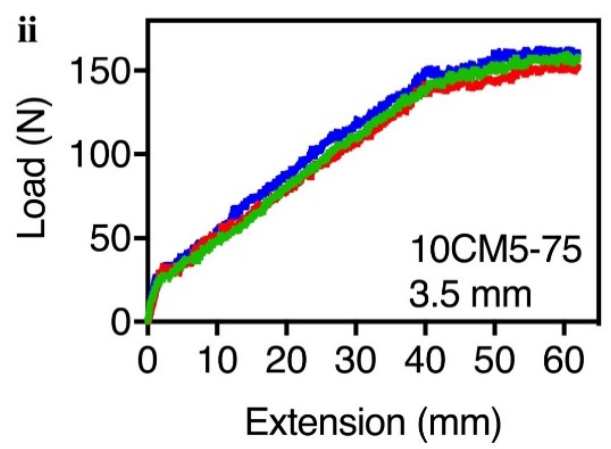

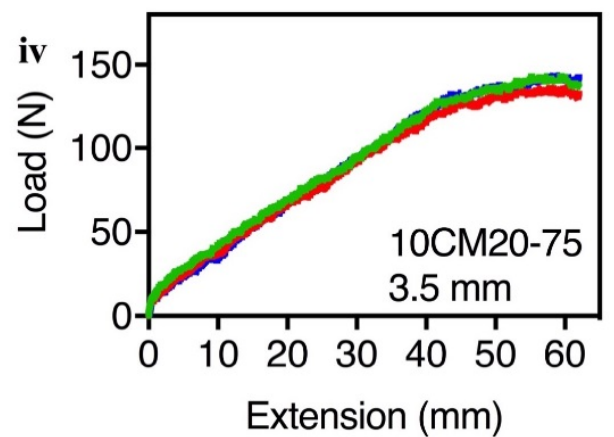

Figure S31. Force profiles of (i) 10CM0-75, (ii) 10CM5-75, (iii) 10CM10-75, (iv) 10CM20-75 through a $3.5 \mathrm{~mm}$ ID catheter.
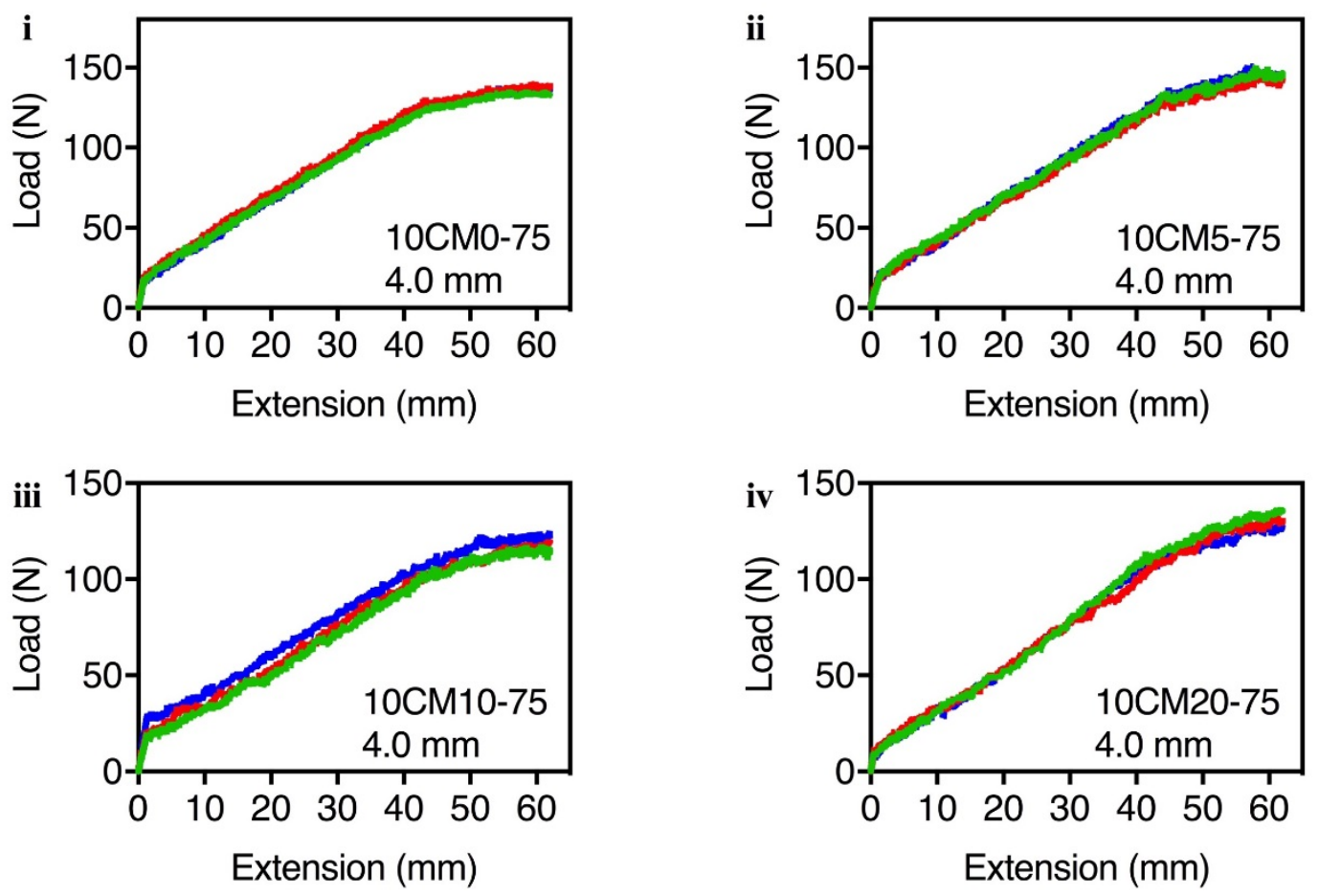

Figure S32. Force profiles of (i) 10CM0-75, (ii) 10CM5-75, (iii) 10CM10-75, (iv) 10CM20-75 through a $4.0 \mathrm{~mm}$ ID catheter. 
Table S1. Manual injectability of composite materials. Materials capable of being injected by manually generated forces are denoted by green. Those not injectable by manual forces are denoted by red.

\begin{tabular}{|l|l|l|l|l|}
\hline \multirow{2}{*}{ Material } & \multicolumn{4}{|c|}{ Catheter diameter $(\mathrm{mm})$} \\
\hline & 2.5 & 3.0 & 3.5 & 4.0 \\
\hline $8 \mathrm{CM} 0-75$ & & & & \\
\hline $8 \mathrm{CM} 5-75$ & & & & \\
\hline $8 \mathrm{CM} 10-75$ & & & & \\
\hline $8 \mathrm{CM} 20-75$ & & & & \\
\hline $10 \mathrm{CM} 0-75$ & & & & \\
\hline $10 \mathrm{CM} 5-75$ & & & & \\
\hline $10 \mathrm{CM} 10-75$ & & & & \\
\hline $10 \mathrm{CM} 20-75$ & & & & \\
\hline $12 \mathrm{CM} 0-75$ & & & & \\
\hline $12 \mathrm{CM} 5-75$ & & & & \\
\hline $12 \mathrm{CM} 10-75$ & & & & \\
\hline $12 \mathrm{CM} 20-75$ & & & & \\
\hline
\end{tabular}
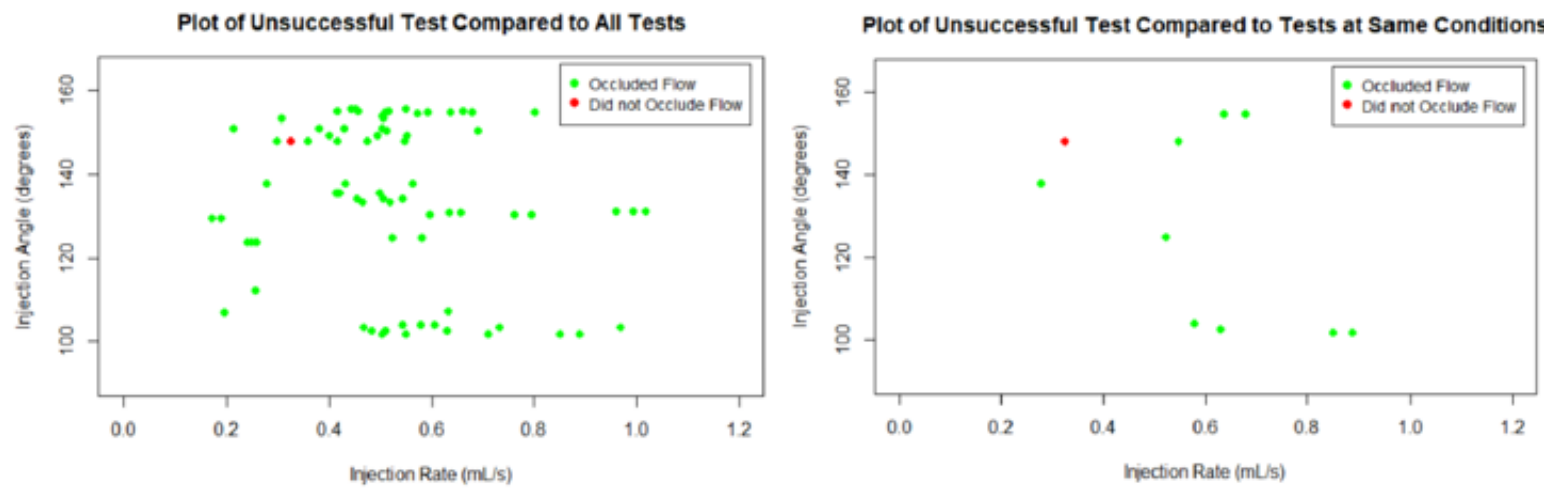

Figure S33. Visualisation of the experimental conditions of the unnecessary test compared to all successful tests (left) and to those tests conducted at the same flow rate and injection location (right). 